\title{
UNSTEADY EXPERIMENTAL AND NUMERICAL ANALYSIS OF A LOW-BOOM INLET
}

\author{
A Thesis Presented to \\ the faculty of the School of Engineering and Applied Sciences \\ University of Virginia \\ In Partial Fulfillment \\ of the Requirements for the Degree of \\ Master of Science in Mechanical \& Aerospace Engineering \\ by \\ Sean Candon
}

May 2014 


\title{
APPROVAL SHEET
}

This thesis

is submitted in partial fulfillment of the requirements

for the degree of

Master of Science

\section{Sean Candon}

AUTHOR

This thesis has been read and approved by the examining committee:

\author{
Eric Loth \\ Adviser \\ Robert Ribando \\ Chair \\ James McDaniel \\ Michael Rybalko \\ Gulfstream Aerospace Corporation
}

Accepted for the School of Engineering and Applied Sciences

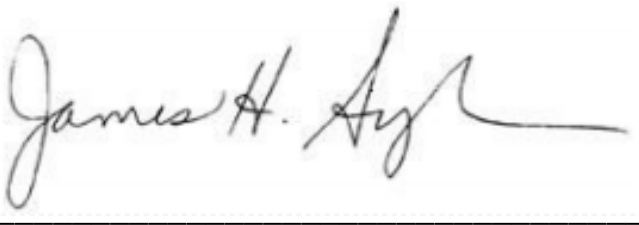

Dean, School of Engineering and Applied Sciences

May 2014 


\section{Acknowledgements}

The experimental results presented in this thesis were collected during tests at the $8^{\prime} \times 6^{\prime}$ supersonic wind tunnel at NASA Glenn Research Center in 2010. I was not part of the experimental effort. I would like to acknowledge the contributions by members of NASA, Gulfstream Aerospace Corporation, the University of Illinois, and the University of Virginia in conducting these experiments. Thank you to all who were involved in the planning and execution of these tests as your efforts have enabled much of the analyses in this thesis.

This project was partially funded by the Virginia Space Grant Consortium (VSGC) through their Graduate STEM Research Fellowship program. I would like to thank Kevin Clark for training and welcoming me into the research group upon my arrival in the summer of 2012. My grateful thanks are extended to Michael Rybalko at Gulfstream Aerospace Corporation for his previous work on the project, for supplying all of the experimental data, and for his continued advice throughout this project.

A special acknowledgement goes to my adviser, Dr. Eric Loth. Your constant guidance, motivation, and knowledge has been instrumental to this project and to my education and growth. Thank you for the opportunity to work on such exciting research.

I would also like to thank my parents for their unconditional lifelong support. Finally, to my brother, Jimmy, thank you for always encouraging me to achieve more and for being such a positive and influential role-model. 


\section{Contents}

Acknowledgements ...............................................................................................................................................................ii

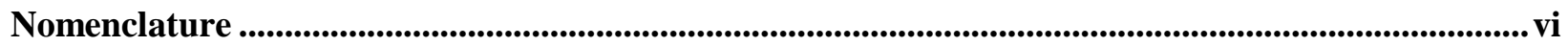

Chapter 1: Research Overview ...................................................................................................................................1

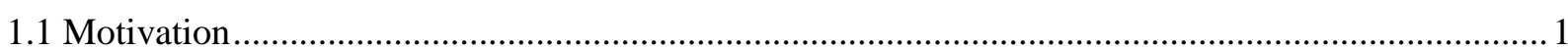

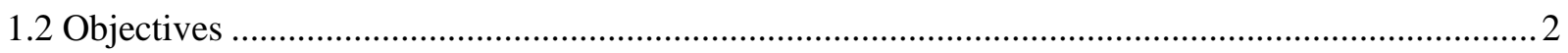



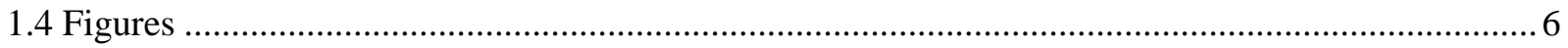

Chapter 2: Unsteadiness at Near-Design Conditions..............................................................................................8

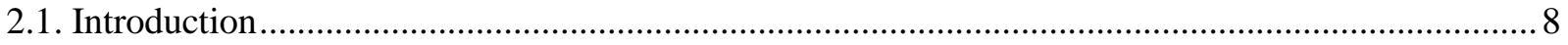

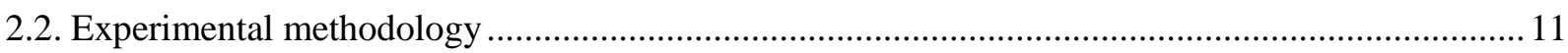

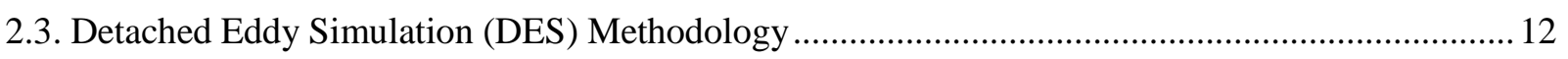

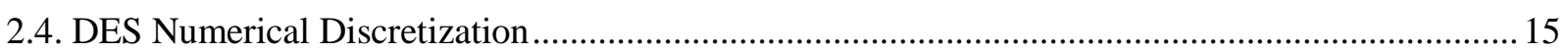

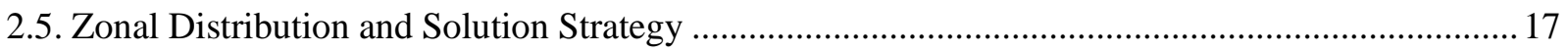

2.6. Defining a Pressure Event.................................................................................................... 19

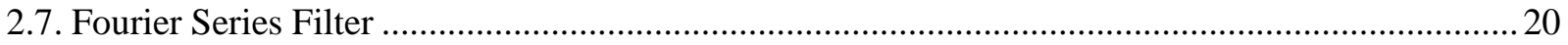

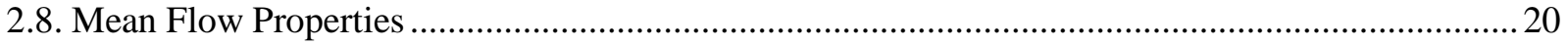



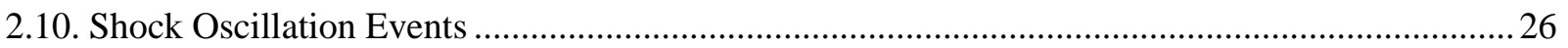

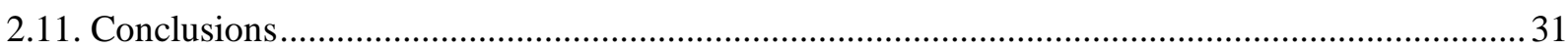

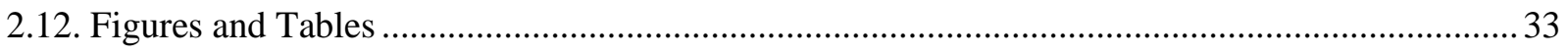

Chapter 3: Acoustically Induced Normal Shock Oscillations............................................................46

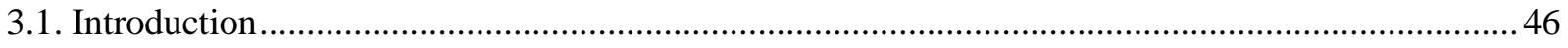

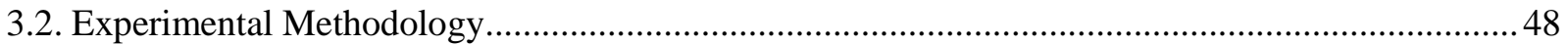

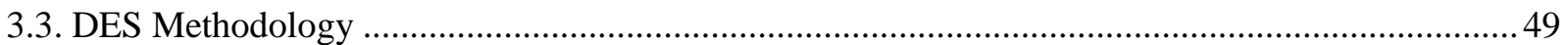

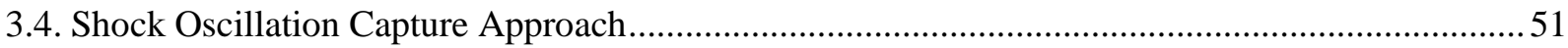

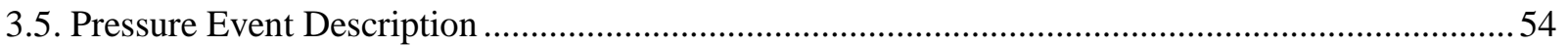

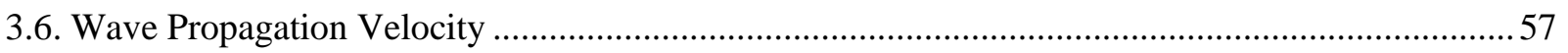

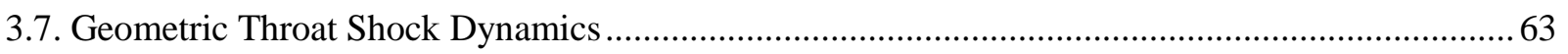

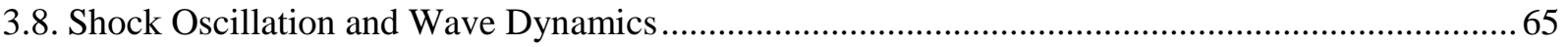

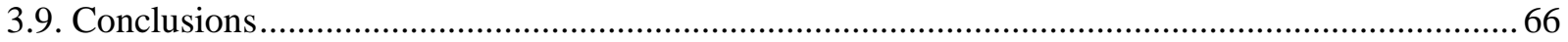

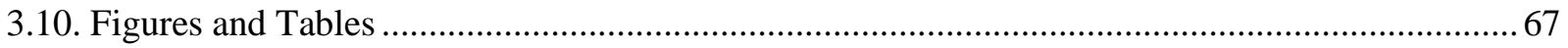

Chapter 4: Spectral Analysis and Mass Flow Rate Effects .........................................................................882 




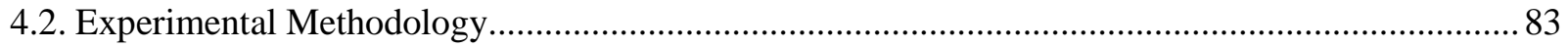

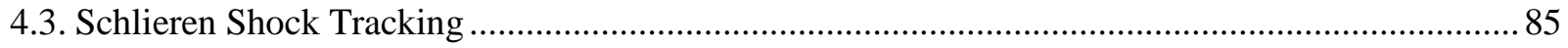

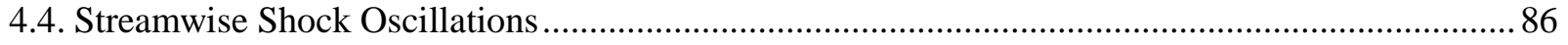

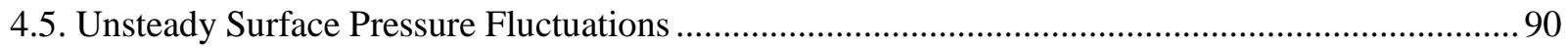

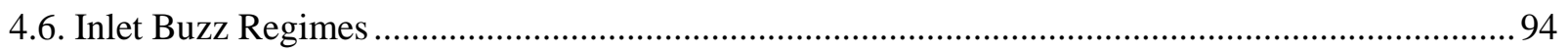



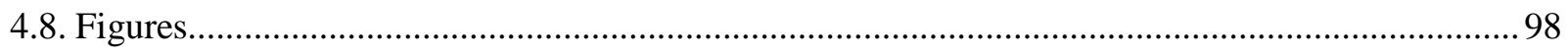

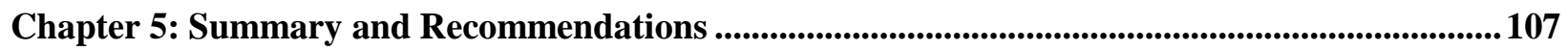

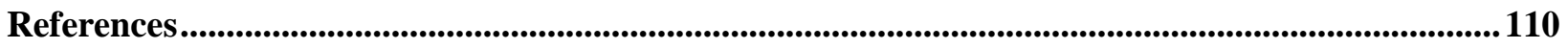




\section{Nomenclature}

$$
\begin{aligned}
& a \quad=\text { Speed of sound } \\
& C \quad=\text { Coefficient } \\
& d \quad=\text { Wall distance } \\
& f \quad=\text { Frequency, function } \\
& L \quad=\text { Length } \\
& N \quad=\# \text { of Eigen-values } \\
& p \quad=\text { Pressure } \\
& \text { MFR = Mass flow ratio } \\
& \text { PSD = Power spectral density } \\
& t \quad=\text { Time } \\
& \text { I } \quad=\text { Intensity } \\
& j=\text { Wall-normal index } \\
& x=\text { Streamwise direction coordinate } \\
& y \quad=\text { Normal direction coordinate } \\
& r \quad=\text { Radial direction coordinate } \\
& \theta=\text { Spanwise direction coordinate } \\
& u \quad=\text { Streamwise velocity component } \\
& v \quad=\text { Normal velocity component } \\
& w \quad=\text { Spanwise velocity component } \\
& S t \quad=\text { Strouhal number } \\
& \Delta=\text { Grid length-scale } \\
& \Lambda=\text { Turbulent integral length-scale }
\end{aligned}
$$




$$
\begin{aligned}
\Omega & =\text { Local mean flow vorticity } \\
\rho & =\text { Density } \\
\varepsilon & =\text { Dissipation } \\
\mu & =\text { Dynamic viscosity } \\
v & =\text { Eddy viscosity } \\
\tau & =\text { Time-scale }
\end{aligned}
$$

\section{Subscripts}

$0=$ Stagnation condition

$\infty=$ Freestream condition

RANS $=$ RANS component

$$
\begin{aligned}
& \text { DES }=\text { DES component } \\
& \text { RMS }=\text { Root mean square } \\
& \text { end } \quad=\text { Wave end } \\
& C B \quad=\text { Centerbody } \\
& \text { comb }=\text { Combined } \\
& \text { cowl }=\text { Inner cowl } \\
& \text { res } \quad=\text { Resolved } \\
& \text { hyb }=\text { Hybrid } \\
& \text { filt } \quad=\text { Filtered } \\
& \text { samp }=\text { Sampling } \\
& \text { sep } \quad=\text { Separation } \\
& \text { sh } \quad=\text { Shock } \\
& \text { start } \quad=\text { Wave start }
\end{aligned}
$$




$$
\begin{aligned}
& \text { thr }=\text { Throat } \\
& \text { peak }=\text { Local peak } \\
& \text { cut }=\text { Cut-off } \\
& \text { AIP }=\text { Aerodynamic Interface Plane } \\
& \text { AC } \quad \text { Acoustic }
\end{aligned}
$$

Superscripts

()$^{\prime}=$ Fluctuation

()$^{*}=$ Normalized

$\overline{()}=$ Time-Average Mean 


\section{Chapter 1: Research Overview}

\subsection{Motivation}

Currently, all commercial and business aircraft are prohibited from supersonic flight over land by federal law. This is due to the produced sonic boom which propagates outward in all directions and is typically quite disruptive at the ground level. This has motivated the design of a "low-boom" demonstrator plane which will ideally illustrate that through careful design, the produced sonic boom may be reduced to a publicly acceptable level. This has involved the redesign of many components, especially those on the external portion of the aircraft. One such component is the engine inlet.

Conners and Howe ${ }^{1}$ of Gulfstream Aerospace Corporation (GAC) have created such a low-boom inlet design which is centered on a relaxed isentropic centerbody compression ramp. Figure 1.1 shows the Mach line field of two types of axisymmetric inlet compression ramps. A general supersonic inlet without low-boom considerations may generate oblique shocks such as those seen in Fig. 1.1a that create a focused region just upstream of the cowl lip. This focused region requires a finite cowl angle (seen in the upper right of Fig. 1.1a) that allows for a certain degree of internal flow turning. However, the greater the cowl angle, the more aggressive the external flow turning. The external flow turning is quite important for low-boom design in that the more aggressive it is, the stronger the external shock is. Therefore, an increase in cowl angle is generally accompanied by a louder sonic boom. By designing the compression ramp in such a way that the oblique shock field becomes defocused, as seen in Fig. 1.1b, there no longer is a need for a finite cowl angle, permitting a reduction to zero or near-zero degrees. This minimizes the external flow turning and therefore also minimizes the sonic boom, satisfying a major low- 
boom design objective. Note that the actual geometries shown in Figs. 1.1a and 1.1b are identical, however, the purpose of the figure was to illustrate differences in the produced oblique shock field. Figure 1.1 was provided by Tim Conners of GAC.

Reducing the cowl angle to zero or near-zero degrees provides significant benefits in overall sonic boom reduction. The effect of the cowl angle is illustrated by nacelle surface pressure contours when using low cowl angle and near-zero cowl angle inlets in Fig. 1.2. Don Howe of GAC provided the images seen in Fig. 1.2 which he generated using computational fluid dynamics (CFD) simulations. Figure 1.2b shows the low cowl angle case which produces a large region of high pressure caused by the external shock from the inlet cowl lip. When reducing the cowl angle to near-zero in Fig. 1.2b, this high pressure region is significantly reduced in both size and magnitude. This shows how the reduced external flow turning of a nearzero angle cowl significantly reduces the produced shock strength, leading to a reduced sonic boom. This is the primary low-boom design modification for the currently investigated inlet.

\subsection{Objectives}

An extensive experimental investigation of the current low-boom inlet was done at the $8^{\prime}$ x 6' supersonic wind tunnels at NASA Glenn Research Center in 2010 that the author was not a part of. Much of the resultant analyses have focused on steady-state data evaluating internal inlet performances such as downstream pressure recovery and distortion. Previous computational studies of the current inlet have also focused on steady-state, investigating other parameters such as the external shock strength. Unsteady aspects of the flow have not been addressed in as much detail as the steady aspects have, especially in the computational realm. Inlet buzz, a phenomena which occurs at low mass flow rates that causes severe streamwise oscillations of the normal 
shock, has been reasonably investigated for the current inlet. However, more modest normal shock unsteadiness observed at higher mass flow rates has largely been uninvestigated.

The objectives of the current study are to investigate the unsteadiness at higher mass flow rates close to on-design, or "near-design," conditions. This unsteadiness is well outside of the inlet buzz regime and is likely caused by other physics. This will be done by analyzing the existing wind tunnel data and by generating unsteady, three-dimensional computational results. A focus is placed on investigating the relationship between shock motion and streamwise propagating waves throughout the downstream diffuser. This will be done by tracking both the normal shock position and pressure wave propagations using unsteady experimental pressure traces. Tracking local pressure maximums between streamwise locations to extract wave velocities is done in Chapter 3. To the author's knowledge, the current study is the first to utilize this method to understand the nature of the observed waves. Computational results will provide additional information on the inlet flow-field that the experimental data cannot. Additional spectral analysis will reveal more on the nature of the dominant waves at near-design conditions.

\subsection{Research Overview}

The experimental wind tunnel data collected at the 8'x 6' supersonic wind tunnel at the NASA Glenn Research Center in 2010 has been analyzed and compared to computational results generated using Detached Eddy Simulations (DES) based on the Nichols-Nelson model. The external compression, axisymmetric inlet includes a relaxed-compression spike followed first by a short subsonic diffuser to the aerodynamic interface plane (AIP) that represents the inflow face of an engine and next by a long diffuser which terminates in the mass flow plug. A 10 degree sector of flow-field is modeled with a 3D structured grid and solved with the WIND-US code. The flow conditions for the experiments and simulations were based on a Mach 1.67 freestream 
coupled with $4 \%$ spillage (higher than the $1.5 \%$ on-design spillage condition). Unsteady pressure measurements were obtained with Kulite transducers at a sampling rate of $5 \mathrm{kHz}$ at six streamwise locations along the inlet centerbody ranging upstream of the normal shock to the AIP. The unsteady surface pressure measurements are used to track motion of the normal shock as well as high pressure waves propagating throughout the diffuser.

A grid resolution study is performed and domain length effects are investigated in Chapter 2. The domain length effects are evaluated using a short domain, which extends only to the AIP and utilizes a fixed back pressure boundary condition, and a long domain which extends from the AIP downstream to the mass flow plug. Local surface pressure time traces are analyzed and compared with experimental traces to reveal wave propagation direction and velocity. The coupling between these waves and normal shock motion is also investigated in Chapter 2 .

Chapter 3 uses the unsteady experimental pressure traces of the $4 \%$ spillage case to more closely analyze and track normal shock motion and compression waves throughout the subsonic diffuser. A second normal shock is observed in the results of Chapter 1. This normal shock and its surrounding flow features are investigated in detail using the same DES approach and experimental data in Chapter 3. Additional information on the unsteady aspects of the flow in this region assists in evaluating the DES approach. In addition, the DES is used to create a summary of the wave mechanics that lead to normal shock oscillations.

Mass flow rate effects are investigated in Chapter 4, ranging from on-design conditions of $1.5 \%$ spillage, to much lower flow rates with $49.5 \%$ spillage. Normal shock stability and surface pressure fluctuations are used to measure these effects at three separate mass flow rates. The unsteady experimental pressure data is used for fluctuation analysis and the shock tracking method and results of Rybalko ${ }^{2}$ is used to quantify normal shock motion. Rybalko ${ }^{2}$ converted 
individual schlieren video frames into intensity bands where the shock location was then extracted. Power spectral density (PSD) distributions are used to determine the energy density throughout the frequency-domain to reveal the dominant mechanisms leading to normal shock and pressure instabilities. Comparisons to acoustic and separation based Strouhal numbers from Dussauge $e t \mathrm{al}^{3}$ and Rybalko ${ }^{2}$ are used to identify the source of unsteadiness in characteristic frequencies.

Final conclusions and summaries of the current work are detailed in Chapter 5, as well as recommendations for future studies. 


\subsection{Figures}

a)

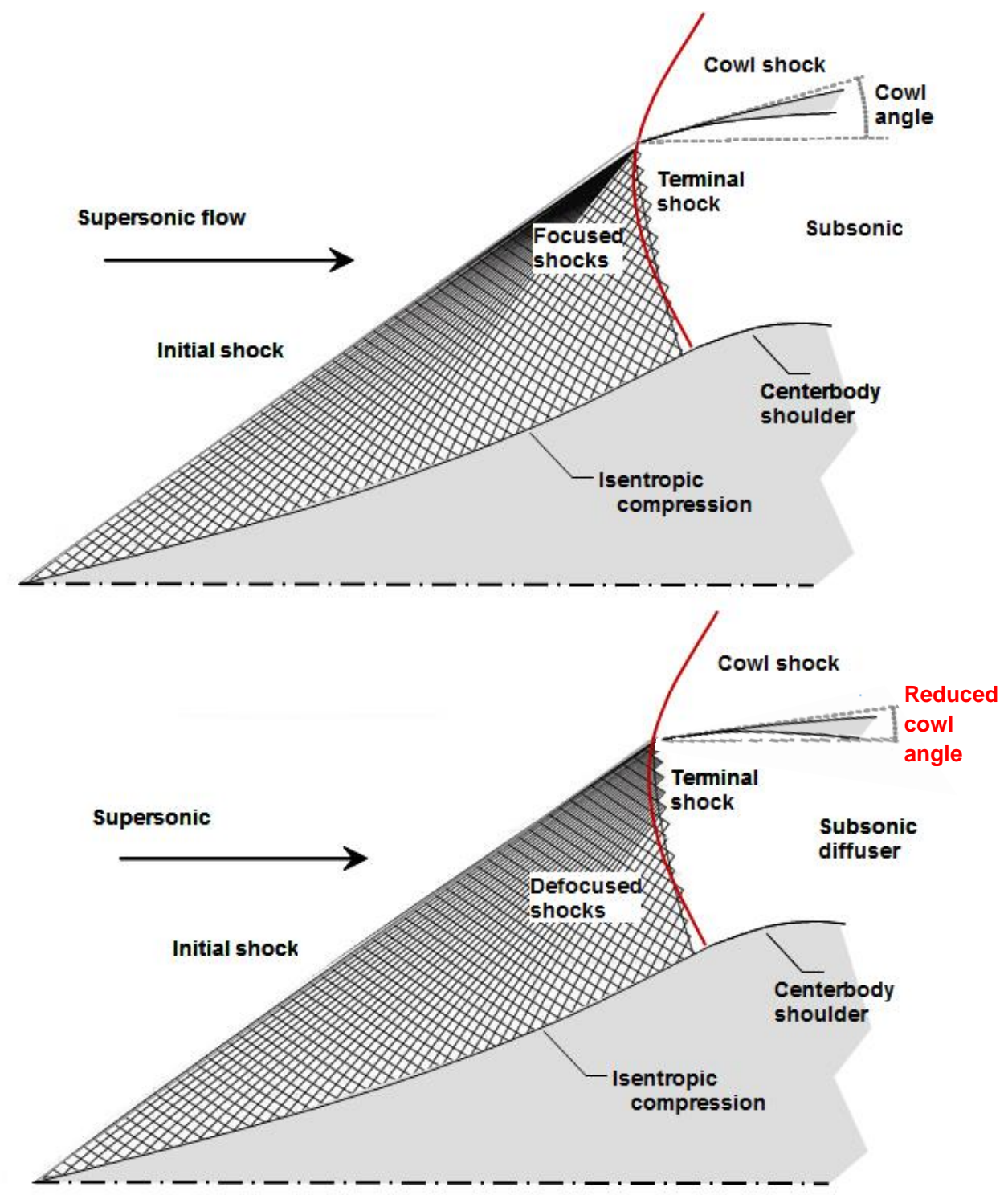

Fig. 1.1: Cross sectional view of a supersonic inlet compression spike illustrating the produces Mach line field with: a) focused oblique shocks at the cowl lip which require a finite cowl angle for relaxation of the internal flow turning, and b) defocused oblique shocks which allow for more aggressive internal flow turning and a near-zero cowl angle. This figure was provided by Tim Conners of GAC. Note that the above geometries are in fact identical in a) and b). The intent of the figure is not to illustrate geometric differences, but rather the differences in the shock field for a low-boom inlet design. 
a)

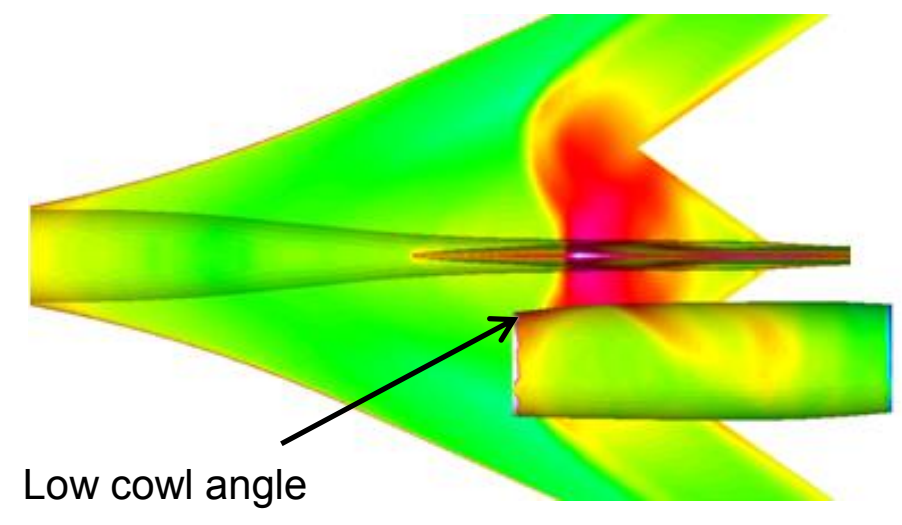

b)

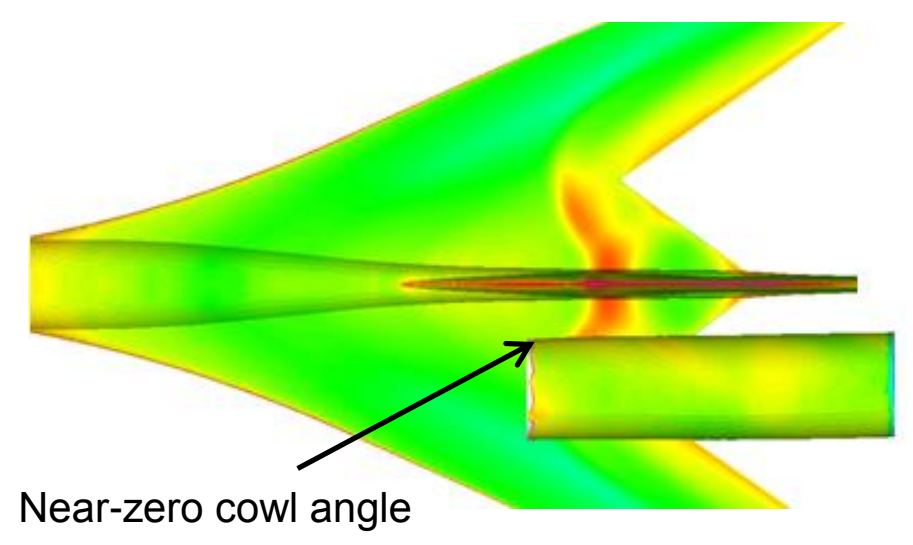

Fig. 1.2: Computational fluid dynamic results of nacelle surface pressure provided by Don Howe of GAC using: a) a low cowl angle inlet and b) a near-zero cowl angle inlet. 


\section{Chapter 2: Unsteadiness at Near-Design Conditions}

\subsection{Introduction}

The low-boom supersonic inlet concept considered in this work is based on a novel design by Conners and Howe ${ }^{1}$. It features a zero-angle cowl, a relaxed isentropic compression centerbody spike, and resulting defocused shocks that form a weaker terminating normal shock on the centerbody. The design was shown to significantly improve supersonic performance and, more importantly, reduce sonic boom overpressure as compared to traditional supersonic inlet designs $^{4,5}$. The specific geometry considered herein is a 12-inch diameter scale model of the full geometry, constructed for supersonic wind tunnel testing in the 8'x6' wind tunnel at NASA Glenn Research Center in Cleveland, OH. A major objective of the study was to examine the effect of vortex generators (VGs) as flow control devices to manipulate the diffuser boundary layer. Several configurations with a variety of VG geometries at two separate streamwise locations were tested. Additional details about the test facility and methodology are found in Hirt

et $a{ }^{6}{ }^{6}$ A photograph of the low-boom inlet model installed in the wind tunnel test section is shown in Fig. 2.1a.

Various flow rates through the inlet were investigated during the wind tunnel tests to note the impact of external flow spillage past the cowl. The mass flow can be normalized by the zerospillage mass flow rate (based on a stream tube stemming from the projected inlet area and freestream conditions) to define the mass flow ratio (MFR). Many flow characteristics important to supersonic inlet design, such as normal shock position and AIP pressure recovery, are strongly dependent on the MFR. The on-design condition corresponded to an MFR of 0.985 (1.5\% spillage) where the external shock and internal flow-field were generally steady and the pressure recovery was high. However, a spillage increase to $4.5 \%$ (MFR of 0.955 ) led to increased 
unsteady phenomena of the pressure field and increased unsteadiness of the shock (based on schlieren video). At very high spillage (more than 50\%), the external and internal flow-field become very unsteady as the inlet flow is governed by violent buzz cycles ${ }^{6}$ and increased unsteadiness $^{7}$. Herein, the focus of the current Chapter is on the more probable near-design conditions where the spillage is only a few percent larger, i.e. at an MFR of 0.955 . The desire to capture normal shock unsteadiness excludes the use of the Reynolds-averaged-Navier-Stokes (RANS) approach for the present computational studies, and therefore an unsteady approach is employed herein that allows simulations at high Reynolds numbers with moderate computational resources.

Numerical simulations of this inlet have previously been conducted for both the design phase and for the post-test analysis phase. Extensive RANS computational studies have been performed on this inlet by Rybalko et al. ${ }^{8}$, Gillen et al. ${ }^{9}$, and Rybalko and Loth $^{10}$ using chimera (overset) grids. A significant focus was to investigate the effect of VGs up- and down-stream of the normal shock. RANS was shown to produce relatively good agreement with the experimental results based on boundary layer rakes as well as centerbody surface pressure distributions. Rybalko and Loth ${ }^{11}$ extended their study to unsteady methods using DES, this time only using the baseline configuration in the absence of VGs. In their paper, they explain the benefits of DES over other time-accurate but more computationally-intensive numerical methods such as Large Eddy Simulations (LES) or Direct Numerical Simulations (DNS). Several orders of magnitude more grid points would be needed for a DNS approach, making it computationally intractable utilizing present computational resources. In contrast, steady-state results were found to converge reasonably for DES grids with about 12 million grid points. In particular, Rybalko and Loth $^{11}$ show increased mean flow agreement with experimental results over previous RANS 
solutions at the on-design MFR. However, the DES solution was seen to be sensitive to grid resolution and cell isotropy and thus care was needed for grid design. As is the case herein, Rybalko and Loth $^{11}$ chose to examine a MFR of 0.955 which has been shown to exhibit increased normal shock oscillations compared to on-design conditions by Rybalko, et al. ${ }^{7}$, and thus would provide a better measure for evaluating unsteady DES performance for the inlet flow. These simulations yielded significant pressure fluctuations occurring in the freestream above the diffuser boundary layer where the turbulent kinetic energy is effectively zero, likely caused by acoustics and shock dynamics. However, their outflow boundary condition (based on a fixed back pressure boundary condition at the AIP) did not allow the capture of possible upstream acoustic waves from the mass flow plug through the cold pipe. This cold pipe is a long diffuser used to connect the inlet model to the mass flow plug (see Fig. 2.1b). One objective of the current study is to include the additional flow-field domain of the cold pipe and mass flow plug for the simulations to capture possible acoustic events, as well as increase grid resolution to better resolve the SWBLI (shock-wave boundary layer interaction). For this purpose, a grid resolution study is performed for the extended, or "DES-long," grid, and results are compared to experiments and the short, or "DES-short", grid which does not include the extension to the mass flow plug. A second objective was to investigate the experimental data to determine the strength and character of unsteady waves which may be responsible for the observed external shock oscillations. This was accomplished by analyzing surface pressure time traces both up and downstream of the normal shock. This study is the first, to the author's knowledge, to utilize unsteady surface pressure traces to reveal wave properties while remaining in the time-domain. Rather than comparing dominant wave frequencies typical of spectral analysis, experimental wave velocity will be extracted to compare with the detailed numerical flow description. 


\subsection{Experimental methodology}

The 8'x6' supersonic wind tunnel at NASA Glenn Research Center can produce Mach numbers up to 2.0, and utilizes perforations to control boundary layer growth along the test section walls. The installed inlet (Fig. 2.1) was based on flight design geometry for the hardware located upstream of the AIP followed by an additional diffuser, cold pipe, and a mass flow plug used to control the mass flow ratio (far right of Fig. 2.1b). The inlet includes a centerbody that terminates with sidewall struts in the diffuser located aft of the AIP. For the present conditions, the Mach number was fixed at 1.67 along with a static pressure and temperature of $31.9 \mathrm{kPa}$ and $217 \mathrm{~K}$, respectively. The inlet scales included a diameter of 12", a length from the spike tip to the AIP of $26.05 "\left(x_{A I P}=26.05 "\right)$ and a length from the spike to the mass flow plug of 174.3". The Reynolds numbers based on the AIP and mass flow plug distances are approximately 12 and 79 million, respectively.

In terms of instrumentation, surface static pressure taps were distributed along the centerbody surface at various streamwise and radial locations, with the majority located on the top dead center (TDC) line. Two boundary layer rakes were located on the inlet centerbody to measure the boundary layer stagnation pressure profiles and help quantify the effect of the VGs. The first rake is placed at a streamwise position 15.5 " downstream of the centerbody spike tip, while the second is located at the AIP. The AIP boundary layer rake contains 14 probes and is located $202.5^{\circ}$ from the TDC line. Unsteady pressure readings are gathered at a $5 \mathrm{kHz}$ sampling rate from eight Kulite pressure transducers located along the centerbody surface using a Dewetron data system. The Kulite taps all fall within $\pm 20^{\circ}$ from the TDC line and are distributed in the streamwise direction. Only one tap per streamwise location is considered in this study, and 
their approximate locations can be seen in Fig. 2.4b. Each reading yields five seconds worth of data, equating to 25,000 values at each tap.

\subsection{Detached Eddy Simulation (DES) Methodology}

Various options exist for generating time-accurate computational solutions including (in order of required computational resources): DNS, LES and DES. The DNS approach resolves all turbulent scales down to the smallest length and time scales with no need for empiricism. However, very fine grids and small time steps are required to capture these physics which leads to high computational demand in terms of both memory and simulation time. This demand coupled with the high Reynolds number associated with this inlet (noted above) would require more than $10^{12}$ grid points, making the DNS approach impractical for this flow. The LES approach reduces the computational expense by implementing empirical models for sub-grid scale turbulence, but still requires substantial memory and computing time to solve high Reynolds number flows. In particular, Rybalko and Loth ${ }^{11}$ estimated (based on LES of a similar flow-field at lower Reynolds numbers completed by Lee ${ }^{12}$ ) that approximately 7.3 billion points would be required to employ LES for the inlet flow upstream of the AIP at the above test condition Reynolds numbers. This too was impractical for the computational resources available for this project.

Therefore, a DES approach, more generally referred to as hybrid RANS/LES approaches, were selected for the present study as they are capable of solving high Reynolds number flows and complex geometries with time accurate unsteady description while requiring reduced computational cost. The hybrid RANS/LES approaches accomplish this by employing a unified approach that operates differently depending on whether the flow is attached or separated. For 
portions of the flow with thin, attached boundary layers, the hybrid approach simplifies to a computationally-efficient RANS method, since this approach has proven reliable in predicting these regions using turbulence models. For regions of separated flow with large-scale turbulence, the hybrid approach reverts to an LES method with a sub-grid scale turbulence model. In between these regions, a blending function is used to ensure smooth transition. The hybrid approach suggests the use of anisotropic grid cells with high aspect ratios at near-wall RANS regions, where refinement is primarily required in the wall-normal direction, and more isotropic cells for LES where wall-normal refinement requirements are similar but similar levels of refinement are now also needed in the spanwise and streamwise directions. Since LES is only being used in separated regions where turbulent length scales are greater, the demanding grid cell isotropy condition can be satisfied with a reasonable number of cells. This yields a significant reduction in the number of grid cells required for DES (compared with LES) and is the primary advantage of DES.

The Nichols-Nelson ${ }^{13}$ DES method chosen for this study employs a blending function based on both grid scales and turbulent length scales. This approach can reduce its sensitivity to grid-induced separation (an issue associated with DES methods in general). A previous study by Rybalko $^{14}$ showed the Nichols-Nelson method accurately predicted velocity deficit and turbulence intensity in the wake of a smooth cylinder when compared to DNS results. This is an important validation since accurate prediction of cylinder wakes requires accurate prediction of the unsteady and time-averaged separation points.

For turbulent transport in the RANS regions, the Nichols-Nelson method utilizes the Menter SST turbulence model which switches from a $k-\omega$ model in the near wall region to a $k-\varepsilon$ model in the freestream. To determine when the approach should transition to an LES 
methodology, the grid dependent length scale and the turbulent dependent length scale are respectively defined as

$$
\begin{gathered}
\Delta=\min \left[d, C_{D E S} \max (\Delta x, \Delta y, \Delta z)\right] \\
\Lambda_{R A N S}=\max \left(6.0 \sqrt{v_{R A N S} / \Omega_{R A N S}}, k_{R A N S}^{3 / 2} / \varepsilon_{R A N S}\right)
\end{gathered}
$$

These length scales are used to calculate the hybrid blending function (which controls transition between RANS and LES regions) as

$$
f_{h y b}=0.5\left(1-\tanh \left[\frac{\pi\left(\Lambda_{\text {RANS }}\right)^{4 / 3}-\pi(2 \Delta)^{4 / 3}}{\left(\Lambda_{\text {RANS }}\right)^{4 / 3}+(2 \Delta)^{4 / 3}}\right]\right)
$$

Its limits are 0 and 1 , with 0 equating to solving with LES and 1 solving with RANS, and anything between being transitional regions. The blending function defined in equation (2.3) uses both length scales, making it dependent on both the grid and the current flow solution, a feature that has been incorporated into more recent DES methods such as Delayed DES ${ }^{15}$. The hybrid turbulence intensity and turbulent eddy viscosity are then solved using

$$
\begin{gathered}
k=k_{r e s}+k_{R A N S} f_{h y b} \\
v_{h y b}=v_{R A N S} f_{h y b}+\left(1-f_{h y b}\right) v_{\Delta}
\end{gathered}
$$

The latter equation uses the sub-grid eddy viscosity $v_{\Delta}$ defined by

$$
v_{\Delta}=\min \left(C_{h y b} \Delta \sqrt{k_{h y b}}, v_{R A N S}\right)
$$

This viscosity alternates between a LES and RANS result based on whether the first or second term is the minimum, where the first term employs the hybrid coefficient $C_{h y b}$ which is set as 0.0854 based on a one-equation $k$ turbulence model. In contrast, the original DES model 
proposed by Spalart et al. ${ }^{16}$ is dependent only on the grid spacing. It is clear from equations (2.4) and (2.5) that when $f_{h y b}$ is equal to 1 , the turbulence intensity and eddy viscosity reduce to

$$
\begin{array}{r}
k=k_{R A N S} \\
v_{h y b}=v_{R A N S}
\end{array}
$$

since $k_{r e s}$ is equal to zero in steady state regions, yielding purely RANS results. When $f_{h y b}$ is equal to 0 the two equations reduce to

$$
\begin{gathered}
k=k_{\text {res }} \\
v_{\text {hyb }}=v_{\Delta}
\end{gathered}
$$

This, therefore, reverts to the LES values.

Consistent with Rybalko and Loth ${ }^{11}$, the WIND-US code, which includes the NicholsNelson hybrid RANS/LES methodology was utilized in the present study. However, the code was modified to improve the performance in terms of shock-capturing. The Superbee total variation diminishing (TVD) limiter was added to the code to preserve monotonicity across the shocks while minimizing dissipation that would degrade the accuracy in the LES regions. Additional changes include time-averaging routines and solution acceleration strategies as described below in Chapter 2.5 .

\subsection{DES Numerical Discretization}

The grid used was a 3D, structured, axisymmetric grid which spanned 10-degrees in the circumferential direction $(\theta)$ with periodic boundary conditions applied in this direction. A TDC slice of the grid can be seen in Fig. 2.2a, along with a mirrored grid image below across the symmetry axis (dashed line) to illustrate the inlet geometry. Fig. 2.2b illustrates the cell 
transition from anisotropic RANS cells upstream of the throat to more isotropic LES cells in regions downstream of the throat, where the flow will generally separate due to shock interaction and become substantially unsteady. By arranging the cells in this manner, the grid dependent length scale can be used to ensure the $f_{h y b}$ value transitions in regions where unsteadiness is expected. A $3^{\text {rd }}$ order upwind biased Roe_over scheme (an implementation from the OVERFLOW code) is used for spatial discretization.

Time discretization and integration were based on previous studies which optimized the approach for DES. A $2^{\text {nd }}$ order approximate factorization alternating direction implicit (AF ADI) method is used for time integration. Constant time steps are used for all DES cases, with values of 1.25e-6 seconds for the DES-long coarse, and 1.0e-6 seconds for the remaining cases presented. These time-steps were selected as the largest for which computational instabilities were consistently eliminated and for which there was negligible influence of time-step on the mean or fluctuating flow-field predictions. To determine the length of time for which timeintegration was required for statistically stationary results, the longest time-scale in the domain was considered. In particular, a full-cycle acoustic time scale $\tau_{A C}$ was defined as the time required for an acoustic wave to propagate from the geometric throat to the mass flow plug and back upstream to the throat using the mean flow speeds and sounds speeds. The acoustic length scale for moving either downstream or upstream is illustrated in Fig. 2.1b. Based on timeaveraged results, it was determined that an integration time of approximately 7 acoustic time scales (0.1 seconds) was reasonable, and therefore the baseline time-averaging was set as 0.1 seconds corresponding to 100,000 time-steps. 


\subsection{Zonal Distribution and Solution Strategy}

A TDC slice of the DES-long fine grid with Mach contours can be seen in Fig. 2.3. It is composed of 20 separate zones, where the first 14 make up the flight inlet geometry and external flow, while the remaining 6 are used for the aft diffuser, cold pipe and mass flow plug region. As shown in Fig. 2.2 and Table 2.1, the grid points were focused on Zones 6-12, where Zones 7 and 8 fall at the same x position and are divided by a circumferential plane as having 5 degrees of span angle each. The specific grids investigated were classified as either "short" or "long" in domain length and either as "coarse", "baseline" or "fine" in grid resolution. The DES-long coarse grid uses a similar zonal distribution with coarser dimensions, however, the DES-short and DES-long baseline (herein referred to as just DES-long) have slightly different configurations. Zones 7 and 8 are combined into one zone, and zones 9 and 10 are combined, along with varying zonal dimensions (all zonal dimensions can be found in Table 2.1). The DESshort grid is only composed of the regions seen in Fig. 2.3a and terminates at the AIP, where a fixed back pressure boundary condition is applied. The DES-short and DES-long cases were the first simulations to be performed in the course of this study, and the zones were split for the remaining simulations in an effort to balance zone sizes and computational load in order to reduce solver run time. In all cases, an identical solution strategy is implemented. The WIND-US code offers a sequencing option that can independently reduce grid resolution by factors of two in each spatial direction and is used throughout the DES solution process. Using a sequencing value of 0 solves at all grid points (full resolution), a value of 1 solves at every other point, and 2 solves at one in every 4 points. Sequencing levels are specified here as $(x r \theta)$ where each integer corresponds to the sequencing level in the corresponding spatial direction. 
First the solution is initialized at freestream conditions of Mach 1.67, static pressure and temperature of $31.9 \mathrm{kPa}$ and $217 \mathrm{~K}$ in zones 1 through 8 , and subsonic conditions in the remaining zones. This initialization creates a normal shock that is in the vicinity of where it eventually settles over time ${ }^{6-11}$. This strategy reduced computation time compared with initializing at uniform sub or supersonic conditions in all zones. Zones 1 and 4 are frozen after being initialized, signified by the "F" in Fig. 2.3a, since they are upstream of the inlet geometry. The flow is then solved for 10k iterations using RANS, with (000) sequencing (full resolution in each direction) for zones 5 and 6, while the remaining zones are sequenced at (222). Rybalko and Loth $^{11}$ investigated the effects of boundary layer transition location and defined a transition region on the centerbody spike to best represent the experimental conditions based on transition modeling and experimental sublimation tests. This same location is employed in the current study by specifying the zone 5 boundary layer to solve as laminar, and where zone 6 , by default, predicts a turbulent boundary layer. The incoming boundary layer in zones 5 and 6 converges to a steady state after $10 \mathrm{k}$ iterations, at which point the zones are frozen to reduce the computational cost. Freezing these zones is justified only by the assumption that the normal shock stays downstream of these zones, which it does in all cases considered. After these 10k iterations, the remaining zones are refined to (111) sequencing for another 10k with RANS, followed by a transition to DES for 0.1 seconds, ensuring any transients due to initialization have passed. Then, 10k time steps of DES at full resolution (000) sequencing are performed to remove transients due to grid refinement. Once these approximately $130 \mathrm{k}$ cycles are complete, time averaging begins for 0.1 seconds in each case. Two sets of averaging, or up to 0.2 seconds, are calculated for the two baseline domain lengths and the DES-long coarse, while three sets are done for DES-long fine. 


\subsection{Defining a Pressure Event}

Since the unsteady pressure measurements indicated shock oscillations, an "event" was defined as when a strong shock perturbation caused the shock to moving significantly upstream from its average position. To define this event quantitatively, the unsteady experimental pressure readings are analyzed for a baseline (no VGs) case. In order to observe normal shock oscillations using a pressure tap, the shock needs to move across the tap. This means the average streamwise position of the normal shock relative to the upstream most Kulite tap will determine if these oscillations will be observed. Therefore, the MFR must be chosen carefully as it is the primary driver of the shock position. Too high of a MFR will result in a shock that sits too far downstream of the tap, which will read only low supersonic pressures, while too low of a MFR will keep the shock upstream of the tap and show only high subsonic pressures. Experimental results at $0.96 \mathrm{MFR}$ are found to best show these pressure fluctuations and are used to compare the experimentally observed shock oscillations with DES.

Significant pressure fluctuations due to shock oscillations are herein referred to as "pressure events." In order to investigate what is occurring at the moment of the shock

oscillation, the moment of the event must be quantified. This is done by recording the moment of the farthest upstream shock position, corresponding to the local pressure peak, and is referred to as $t_{\text {sh,peak }}$. To extract a similar pressure trace from the DES, data is output at every time step at a streamwise location of $x / x_{A I P}=0.29$. This high resolution tap is placed slightly farther upstream than the experimental location $\left(x / x_{A I P}=0.33\right)$ because the MFR of the DES is on average equal to 0.94 , moving the normal shock slightly upstream compared to the experimental data with MFR $=0.96$. The remaining DES pressure traces are collected every 200 time-steps at 
streamwise locations of $x / x_{A I P}=0.37,0.52,0.67,0.83$ and 0.98 , which are identical to the experiments.

\subsection{Fourier Series Filter}

To consistently compare the experimental and DES pressure traces with respect to lowfrequency events associated with shock-oscillations, the high-frequency events associated with fine-scale turbulence were filtered out. This is done by representing the perturbation pressure $\left(p^{\prime}=p-\bar{p}\right)$ as a Fourier series to act as a low-pass filter and remove high frequency waves. The cut-off frequency of the filter $\left(f_{c u t}\right)$, or the maximum passable frequency, is defined as the number of Eigen-values $(\mathrm{N})$ used divided by the length of the time window being filtered $\left(t_{f i l t}\right)$.

$$
f_{\text {cut }}=N / t_{\text {filt }}
$$

When comparing experimental and DES data, a filter cut-off frequency of $f_{c u t}=200 \mathrm{~Hz}$ is used for both data sets. This cut-off frequency is reduced to $70 \mathrm{~Hz}$ when the propagation velocity of only the lower frequency DES waves is closely examined.

\subsection{Mean Flow Properties}

A comparison between instantaneous and time averaged Mach contours can be seen in Fig. 2.4 from the DES-long fine case. The defocused oblique shocks due to the isentropic compression field of the centerbody beginning at the spike tip gradually decrease the Mach number as the flow approaches the normal shock. Once passing through the normal shock, the sudden pressure increase and deceleration significantly reduces the Mach number. The instantaneous contour in Fig. 2.4a shows the lambda structure at the base of the normal shock consisting of an oblique and normal shock in series caused by the SWBLI. This causes the centerbody boundary layer to separate shortly after passing through the lambda structure 
illustrated by the low Mach region in blue. The flow then locally re-accelerates to supersonic leading into the geometric throat due to flow turning, where a second normal shock is present to decelerate the flow into the diffuser. Turbulent structures are visible in the instantaneous Mach contours being shed downstream of the throat. The boundary layer is seen to again separate downstream of the geometric throat, with a recirculation zone present.

In order to evaluate the grid dependency, a resolution study is performed on the DESlong grid at coarse (8.6M points), baseline $(14.1 \mathrm{M})$, and fine $(16.7 \mathrm{M})$ configurations. Stagnation pressure boundary layer profiles taken at the AIP are used to compare the three resolutions and can be seen in Fig. 2.5 along with experimental results, where

$$
r^{*}=\left(r-r_{C B}\right) /\left(r_{\text {cowl }}-r_{C B}\right)
$$

This non-dimensional radial distance represents the height off the centerbody surface normalized by the clearance between the centerbody and inner cowl surface. Thus, it varies from 0 at the centerbody to 1 at the inside cowl surface. Figure 2.5 a illustrates time period convergence of the mean stagnation profile of the DES-long fine case from 10k to 30k time steps, indicating strong convergence relative to the $100 \mathrm{k}$ time-step overall time integration used for baseline averaging (0.1 seconds). In Fig. 2.5b, all three grid resolutions, along with DES-short, are shown in comparison to experimental values for three similar MFR values. The predictions show good agreement in the lower $50 \%$ of the boundary layer and the boundary layer height is accurately predicted with good agreement at the last three probes extending into the freestream. Small differences arise at the top $50 \%$ where higher refinement and longer domains produce a fuller profile. This could be caused by better capturing the lambda shock details which reduces the stagnation pressure losses. However, in all cases, the boundary layer profile is over predicted 
compared to the experimental results, indicating that the flow separation may be under-predicted or the recovery length over-predicted.

Fig. 2.6a shows static pressure contours for the DES-long fine case with arrows indicating experimental streamwise Kulite tap locations above with a plot of TDC centerbody surface static pressure distributions below. The normal shock is clearly illustrated by a strong pressure gradient and the expansion region followed by the second normal shock at the throat is also visible. The TDC surface static pressure distributions also indicate the two separate shocks with sudden pressure increases at $x / x_{A I P}=0.3$ and $x / x_{A I P}=0.35$ in Fig. 2.6a. The experimental local minimum pressure due to the expansion region is lower than the predicted DES values, which would be indicative of an under-predicted separation bubble. Increasing grid resolution shows a decrease in the minimum pressure of the expansion region, suggesting that further refinement may better predict the separation region upstream of the throat. Throughout the remainder of the diffuser the DES predictions are generally higher than the experimental data until far downstream, indicating the pressure recovery is somewhat over-predicted. Therefore, all the main features are captured, but there are some quantitative differences.

The skin friction coefficient serves as a normalization of the wall shear stress and is defined by

$$
C_{f}=\mu\left(\frac{\partial u}{\partial y}\right)_{j=1} / \frac{1}{2} \rho u_{\infty}^{2}
$$

The velocity gradient is calculated using the first off-wall grid point. Time-averaged streamwise skin friction coefficient distributions for both baseline resolution domain lengths are seen in Fig. $2.6 \mathrm{~b}$, with negative values indicating reverse flow regions. Consistent with the Mach contours in Fig. 2.4, the skin friction coefficient shows small separated regions just upstream of the throat at 
approximately $x / x_{A I P}=0.33$ in both domain lengths, characteristic of normal SWBLIs. The DES-short predicts a second separation downstream of the throat, which the DES-long case does not. This is due to a more developed averaged boundary layer predicted by the DES-long after reattachment downstream of the normal shock, indicated by the higher skin friction coefficient value around $x / x_{A I P}=0.35$ in Fig. 2.6b. Based on the differences noted in Fig. 2.6a, it is expected that the experimental conditions did not include this second separation (seen only in the short domain results), at least in an average sense.

\subsection{Fluctuating Flow Properties}

Figure 2.7a shows iso-surfaces of the Lambda-2 criterion to illustrate turbulent structures near the centerbody surface taken from instantaneous DES-long fine results. Three-dimensional turbulent structures are found just upstream and just downstream of the geometric throat. The upstream turbulent structures are products of the SWBLI that occurs at the base of the normal shock and causes a small separation bubble, illustrated by negative skin friction coefficient values in Fig. 2.6b at $x / x_{A I P}=0.33$. Motion of the normal shock will cause the separation point to move in the streamwise direction leading to significant unsteadiness and the small scale turbulent structures seen in Fig. 2.7a. Figure 2.7b zooms in on just downstream of the throat, defined by the red box in Fig. 2.7a. Figure 2.6b does not indicate separation downstream of the throat for the DES-long fine case meaning these structures are occurring in a flow which is onaverage, attached. This indicates that the region downstream of the throat illustrated in Fig. 2.7b likely exhibits a blended RANS/LES region with a $f_{h y b}$ between 0 and 1 . The three-dimensional turbulent structures disappear shortly after moving downstream of the black arrow in Fig. 2.7a, indicating a RANS solution downstream of this point and a fully attached boundary layer. The 
over prediction of the stagnation pressure rakes at the AIP in Fig. $2.5 \mathrm{~b}$ could be caused by the additional RANS mixing just downstream of the throat in the blended region. This would allow the boundary layer to redevelop (illustrated by absence of coherent turbulent structures downstream of arrow in Fig. 2.7a) prematurely resulting in a fuller profile. Further investigation using methods to integrate pure LES in this downstream portion is recommended for future studies.

Pressure fluctuations are compared between DES and experimental data in Fig. 2.8 using the root-mean-square (RMS) values. Figure 2.8a shows averaged DES-long fine AIP rake results of three separate time-averaged data sets taken in series, each integrating over 0.1 seconds, or just over 7 acoustic sweeps of the domain. An overall average of the three sets is shown in black. Experimental Kulite data for MFR $=0.96$ is shown using symbols. The experimental data set is composed of five seconds of pressure traces, or approximately 360 acoustic sweeps of the domain. Using the RMS value of pressure fluctuations for the whole 360 sweeps will capture many more significant pressure events, such as those seen in Fig. 2.9, than the seven acoustic sweeps that the DES averaged sets capture. To better compare DES to experiments, additional experimental $p_{R M S}^{\prime}$ values are calculated using a similarly sized time integration window to the DES. The DES-long fine case produces a shock oscillation causing a pressure event seen in Fig. $2.10 \mathrm{~b}$, so a similarly shaped event is sought out in the experimental data to collect a local $p_{R M S}^{\prime}$ value spanning $t / \tau_{A C}=0-7.1$. The specific experimental event chosen can be seen in Fig. 2.9b. This local $p_{R M S}^{\prime}$, represented by squares, shows good agreement with the first averaging set from DES-long fine seen in red in Fig. 2.8a, which encompasses the shock oscillation in Fig. 2.10b. Therefore, the pressure fluctuations downstream in the diffuser are being reasonably predicted during one shock oscillation event. When comparing the DES rake results to the full time 
averaged experimental case from $t / \tau_{A C}=0-356$ represented by circles, the DES under-predicts the fluctuations in all averaged sets indicating that a much longer integration period is required to capture the quantitative pressure unsteadiness. As the DES continues to integrate in time and collect averaged data sets, the pressure fluctuations continuously decrease. This is due to the events seen in Fig. 2.10b, which repeatedly occur approximately once per data set, but with decreasing amplitude each time. It is possible that the events of similar magnitude seen in Fig. $2.10 \mathrm{~b}$ occur at a longer time scale than the current time integration extends to; meaning future studies with longer integration time for averaging may capture more of these events. Figure $2.8 \mathrm{~b}$ illustrates $p_{R M S}^{\prime}$ as a function of streamwise distance. The DES data sets that are presented in this plot encompass only the first 0.1 seconds of time averaging. The experimental data is presented using the same two time-averaging windows $\left(t / \tau_{A C}=0-356\right.$ as circles, $t / \tau_{A C}=0$ 7.1 as squares) to compare with DES. Consistent with Fig, $2.8 \mathrm{a}$, the $7.1 \tau_{A C}$ experimental timeaveraging window shows fewer pressure fluctuations than the full $t / \tau_{A C}=0-356$ case. Between $x / x_{A I P}=0.3$ and 0.4 there are four local maximums predicted by the DES-long case seen in solid blue. These peaks correspond to the upstream and downstream edges of the lambda shock produced by the SWBLI. The first two peaks occurring at approximately $x / x_{A I P}=0.3$ and $x / x_{A I P}=0.34$ are the edges of the lambda from the normal shock, which goes through an oscillation, causing the upstream (oblique shock) and downstream (normal shock) edges to register high pressure fluctuations, while the region in between these two peaks never experiences a shock edge and is therefore isolated from major pressure fluctuations. The DESshort does not show significant peaks in this region, illustrating shock steadiness. The contradictory results between DES-short and DES-long indicate a significant sensitivity to domain length of the surface pressure fluctuations. The experiments show high pressure 
fluctuations at the $x / x_{A I P}=0.33$ tap, which corresponds to the upstream edge of the normal shock lambda. The difference between the experimental value at this point, and the first significant peak in the DES-long data at $x / x_{A I P}=0.3$ illustrates the streamwise difference in shock location due to the slight MFR differences. Peaks also occur near the geometric throat at $x / x_{A I P}=0.37$ for both domain lengths, caused by a second shock located in this region, which is illustrated in both Figs. 2.4 and 2.6a. Surface pressure fluctuations decrease in magnitude with the increased resolution of the DES-long fine case, especially near the peaks caused by the throat shock. This is consistent with the predicted separation bubble downstream of the throat by the baseline resolution in Fig. 2.5b which causes increased pressure fluctuations. This further suggests that increasing the grid resolution may lead to over activation of RANS in separated regions causing over prediction of boundary layer fullness and increased stability. The remaining taps in the diffuser are under predicted by the DES-long cases when comparing to the full timeaveraged $t / \tau_{A C}=0-356$ set. When comparing to the experimental $t / \tau_{A C}=0-7.1$ set, both DESlong resolutions show reasonable agreement with the diffuser taps, while DES-short predicts fewer fluctuations.

\subsection{Shock Oscillation Events}

The previously discussed results have shown that the DES-long case appears to capture some instabilities that the DES-short case does not. It was hypothesized that the DES-long case would capture acoustic waves reflecting from the downstream mass flow plug. To investigate this, surface pressure traces are analyzed to monitor potential wave propagations. Examining the pressure traces of the upstream most Kulite tap located at $x / x_{A I P}=0.33$, pressure events are clear and can be seen in Fig. 2.9 normalized by the free stream pressure as a function of time normalized by the acoustic time-scale, i.e. $t / \tau_{A C}$. A single-peak event is shown in Fig. 2.9a 
indicating a Gaussian wave distribution that occurs over a dimensionless time interval of approximately 1 , while double- and triple-peak events appear to occur over slightly longer times and are seen in Figs. 2.9b and 2.9c. The equivalent upstream DES pressure trace can be seen in Fig. 2.10a for DES-short and 2.10b for DES-long fine, with both Fourier filtered data in blue and unfiltered data in red. Comparing just the unfiltered DES curves for $t / \tau_{A C}<2.5$ (for which there are not significant shock events), the DES-long shows greater amplitude fluctuations, indicative of a more turbulent flow consistent with Fig. 2.8b. Figure 2.10b shows a significant pressure event beginning at $t / \tau_{A C}=2.5$ lasting for approximately a time of $2 \tau_{A C}$, exhibiting two peaks with the filtered curve. The DES-short data fails to produce this kind of low frequency event. Since this event seen in Fig. 2.10b exhibits double peaks, it is compared to the experimental double peak event of Fig. 2.9b.

Propagation direction can be determined to better characterize the wave by examining the remaining streamwise pressure taps at the time of the observed pressure event. Due to the extreme pressure jumps from normal shock oscillations, the upstream tap shows significantly greater fluctuating pressures when compared to the downstream, fully subsonic taps. To better compare experimental and DES data at the three tap locations, pressure events are normalized by their corresponding $p_{R M S}^{\prime}$ values to create curves of approximately equal amplitude. To also compare at consistent times, a normalized time variable $t_{s h \text {,peak }}^{*}$ is introduced and defined as

$$
t_{\text {sh,peak }}^{*}=\left(t-t_{\text {sh,peak }}\right) / \tau_{A C}
$$

This shifts the time origin to the time of the shock oscillation peak $\left(t_{\text {sh,peak }}\right)$ at $x / x_{A I P}=0.33$. Figure 2.11a shows the experimental event, both with unfiltered points and Fourier filtered, at different pressure tap locations given by $x / x_{A I P}=0.33,0.52$, and 0.98 . The filtered DES event 
curves are shown in Fig. 2.11b at identical streamwise locations. The overall shapes of the events are comparable at each location, and both DES and experiments show a time phase shift between local peaks. When considering just the local peaks in Fig. 2.11a between $t_{s h, p e a k}^{*}=-0.6$ and 0.1 (highlighted with black arrows), the downstream most tap (blue) peak occurs first in time, followed by the midstream (green), and finally the upstream tap (red) peak. The time shift of peaks demonstrates that the experimental pressure event begins downstream and propagates upstream. This same shift is also seen in the DES in Fig. 2.11b when comparing local peaks between streamwise locations.

Since the pressure events were found to begin downstream and propagate upstream to the normal shock, the velocity of these events is investigated using both experiments and simulations. Event times are taken at the same streamwise locations corresponding to the experimental Kulite taps. The DES traces seen in Fig. $2.11 \mathrm{~b}$ use $f_{c u t}=200 \mathrm{~Hz}$ at the downstream taps, but to better quantify these pressure fluctuations into a single event, a lower value of $f_{\text {cut }}$ is required. Figure $2.12 \mathrm{a}$ shows a cyan curve using an $f_{c u t}=70 \mathrm{~Hz}$ filter which succeeds in converting the event into a single peak, as well as a comparison of other $f_{\text {cut }}$ values and the unfiltered data. Using filtered pressure traces using $f_{c u t}=70 \mathrm{~Hz}$ at each streamwise tap, the local maximum peak times $\left(t_{A I P, \text { peak }}\right)$, indicated by a black arrow in Fig. 2.12a, are recorded. To refer the event to when pressure peaked downstream at the AIP, a normalized time can be referenced as

$$
t_{A I P, p e a k}^{*}=\left(t-t_{A I P, p e a k}\right) / \tau_{A C}
$$

This referencing creates a normalized time whose positive values correspond to an upstream running wave starting at the downstream-most location. The time values are plotted against streamwise distance in Fig. 2.12b, along with DES-long fine predicted acoustic wave 
propagation. The predicted wave propagation is determined by integrating the local upstream running acoustic velocity $(u-a)$ at each grid cell across the subsonic diffuser length. This integration is done at various wall-normal heights and an average over the whole wall-normal span is seen in red. The times of the events (symbols) all fall relatively close to the averaged upstream running acoustic velocity curve, with the greatest deviation seen at the upstream-most location. This is to be expected, as this is the most unsteady region where transonic velocities are present. This agreement confirms that the pressure waves that propagate upstream are moving at an acoustic velocity, further supporting the capture of unsteady acoustic events using the DESlong domain. The experimental value at $x / x_{A I P}=0.37$ is not included due to its near-throat placement and the corresponding pressure trace complexity caused by the second shock. This region exhibits transonic velocities, preventing acoustic wave propagation and thus falls out of the scope of the current study.

The number of pressure events over known time spans can be used to calculate the estimated frequencies of the events. The experimental pressure trace shows approximately 100 pressure events defined by a pressure fluctuation that exceeds $p / p_{\infty}>2$ over a five second span, equating to a frequency of $20 \mathrm{~Hz}$. The DES exhibits one event over a span of 0.1 seconds for a $10 \mathrm{~Hz}$ frequency. Analysis of Rybalko ${ }^{17}$ describes normal shock oscillation frequency dependence on the separation length, approximated in Fig. 2.1a for the current flow-field. He related a separation based Strouhal number of 0.035 to normal shock oscillation frequency as well as freestream velocity defined as

$$
S t_{\text {sep }} \approx 0.035=\frac{f_{\text {sep }} L_{\text {sep }}}{u_{\infty}}
$$


Calculating the separation length from the skin friction coefficient distribution in Fig. $2.5 \mathrm{~b}$ and rearranging (2.16) will yield an estimated separation based frequency of

$$
f_{\text {sep }}=\frac{(0.035)(493 \mathrm{~m} / \mathrm{s})}{0.0169 \mathrm{~m}}=1021 \mathrm{~Hz}
$$

This result is orders of magnitude greater than both the experimentally and computationally observed frequencies, suggesting it is unlikely to be the dominant instability. The acoustic based frequency can be calculated using a similar derivation with an acoustic Strouhal number equal to 1.

$$
S t_{A C} \approx 1=f_{A C}\left(\frac{L_{A C}}{a+u}+\frac{L_{A C}}{a-u}\right)=f_{A C}\left(\frac{3.03 \mathrm{~m}}{474+136 \mathrm{~m} / \mathrm{s}}+\frac{3.03 \mathrm{~m}}{474-136 \mathrm{~m} / \mathrm{s}}\right) ; \Rightarrow f_{A C}=71.78 \mathrm{~Hz}
$$

This frequency is greater than the observed DES and experimental frequencies but shows significantly better agreement when compared to the estimated separation based frequency. It is unclear if the observed waves are indeed acoustic based on these estimated frequencies, however, it is more likely that the instability is driven by acoustic modes rather than by the separation length.

Analysis of grid resolution effects shows that greater refinement leads to increased RANS mixing in regions of unsteadiness such as the SWBLI induced separation bubble and just downstream of the geometric throat. This suggests that further grid refinement will likely not improve agreement with experimental data and may in fact diminish agreement. The DES appears to fall short of accurately predicting the reattachment region, therefore higher fidelity methods are recommended in these regions. Using 100k time-steps at fine resolution, two weeks are required for the current computational resources to compute 0.1 seconds of data. It was not practical for the authors to simulate $5 \mathrm{M}$ time-steps to equal the 5 seconds of the experimental 
data, however, future studies with sufficient resources to do so are recommended for a better comparison of the fluctuating values. This is especially needed for further DES wave frequency analysis. The presence of a second shock wave at the geometric throat leads to increased complexity at the throat region which was largely skipped in the current study. If this throat shock exhibits similar dynamics as the normal shock then it will have a significant impact on the downstream flow-field. A detailed investigation of this throat shock dynamics is recommended for future studies.

\subsection{Conclusions}

A DES methodology was applied to a low-boom inlet flow field at off-design mass flow conditions using various grid refinement levels and domain lengths. Mean flow results show only slight deviations between grid refinement levels, however, more significant deviations are seen with domain length differences. An over prediction of the downstream boundary layer is amplified with increasing grid resolutions, likely stemming from increased RANS mixing at the separated regions near the geometric throat. Dynamic flow characteristics show a stronger dependence on domain length but only a slight dependence on grid resolution. The short domain case predicts significantly fewer streamwise surface pressure fluctuations compared with the long domain, as well as evidence of a stable normal shock. This justifies using an extended domain including the cold pipe and mass flow plug in order to capture additional unstable flow features.

By monitoring the normal shock position by means of pressure time traces, the long domain case was able to capture unsteady events that the short domain failed to. A pressure wave is seen to originate in the downstream region of the diffuser and propagate upstream at approximately acoustic velocity, causing the normal shock to move upstream from its average 
position. Similar pressure waves are observed in experimental data and their shapes agree reasonably, as do their downstream origins. Further investigation of the experimental pressure waves is required to more specifically characterize the compression waves. Wave frequency analysis revealed that the separation based instability is likely not the instability leading to shock motion but also did not conclusively support acoustic modes as an alternative. Only one pressure wave is captured using 0.1 seconds of DES, while the experimental data set contains approximately 100 in a span of 5 seconds. Future studies should capture many of these pressure events using DES to best compare unsteadiness between experiments and computations. 


\subsection{Figures and Tables}

a)

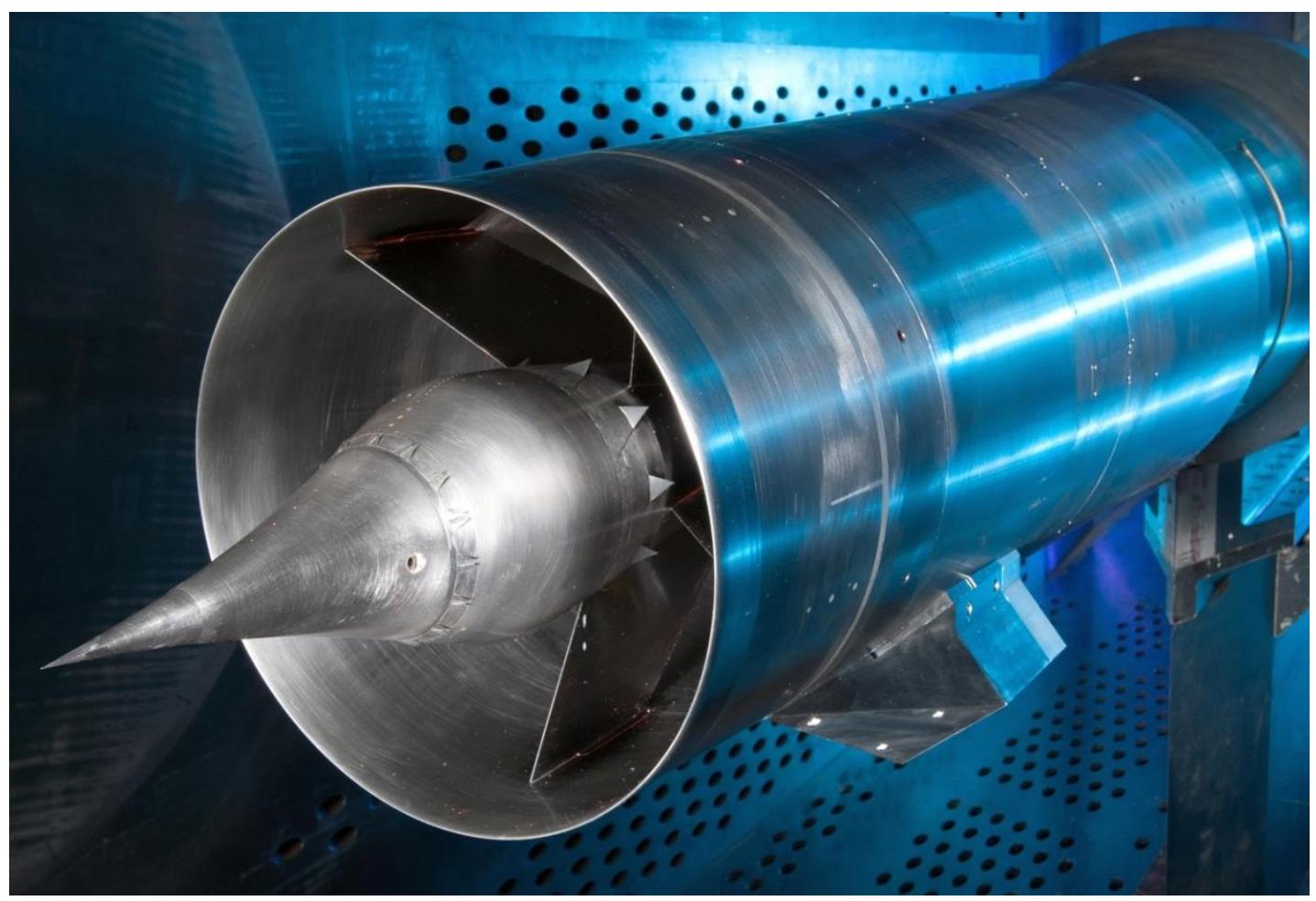

b)

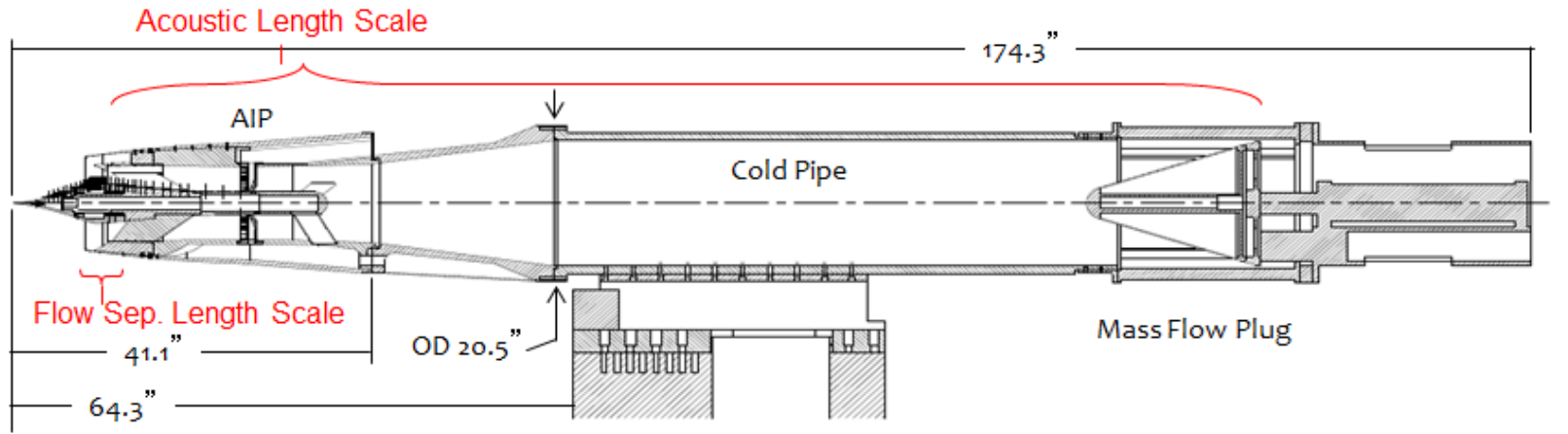

Fig. 2.1: a) Low-boom inlet installed in the 8'x6' Supersonic Wind Tunnel test section at NASA Glenn Research Center. Split-ramp (upstream) and ramp (downstream) VGs are visible on the inlet centerbody. Photograph courtesy of NASA. b) Inlet schematic showing cold pipe and mass flow plug as well as characteristic length scales. 

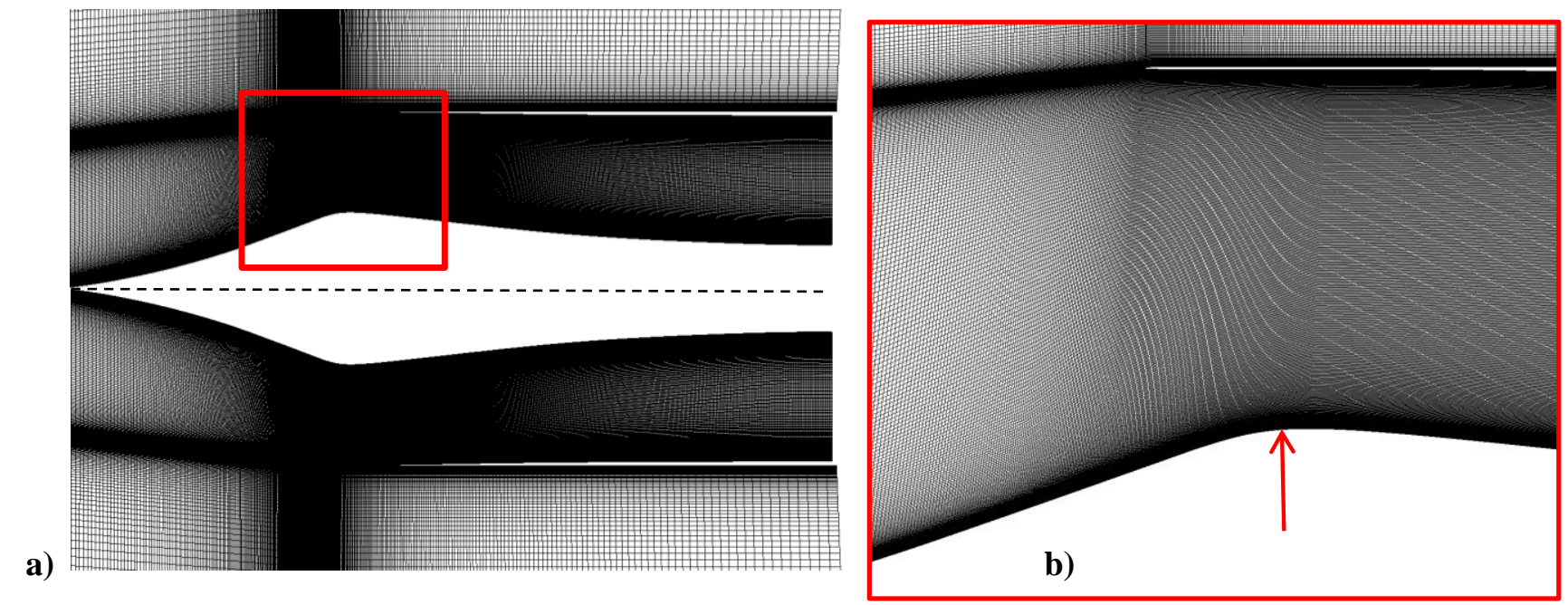

Fig. 2.2: TDC slices of the baseline computational mesh of the a) inlet region showing the grid mirrored across the symmetry axis (dashed line) and b) a close up of the geometric throat region illustrating node distribution where downstream throat location (minimum cross-sectional area location) is at $x_{t h r} / x_{A I P}=0.37$ " and is shown by a red arrow. 


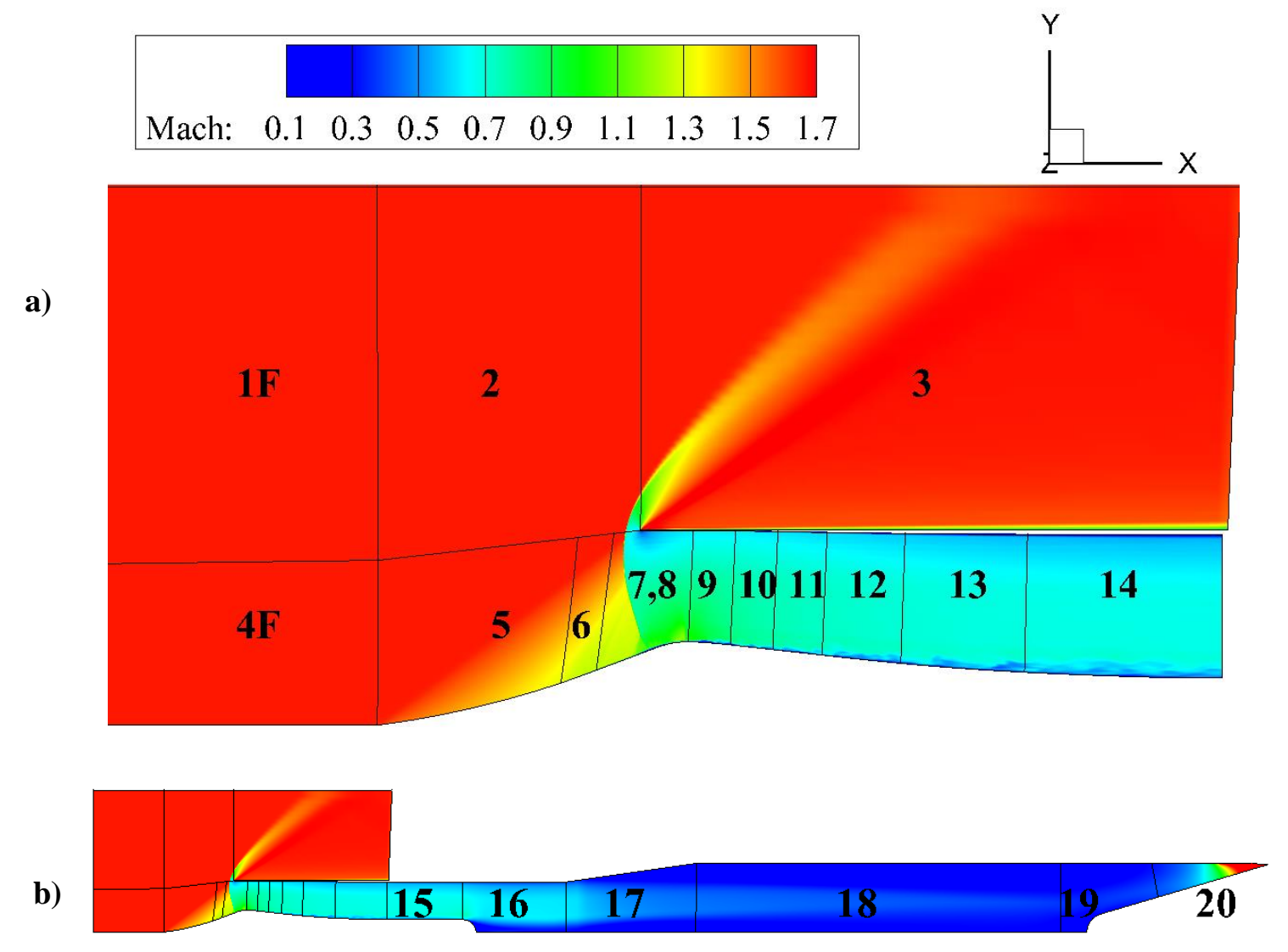

Fig. 2.3: Zonal distribution for the a) DES-short and b) the DES-long grids with frozen freestream zones signified by an F. Zone 5 is solved with a laminar boundary layer and transitions the grid spacing from a RANS type at the centerbody spike to a DES type at zone 6 where the boundary layer is set to become turbulent. Zones 7 and 8 share a spanwise boundary. The baseline DES-long grid combines zones 7 and 8 into a single zone, as well as zones 9 and 10 . 


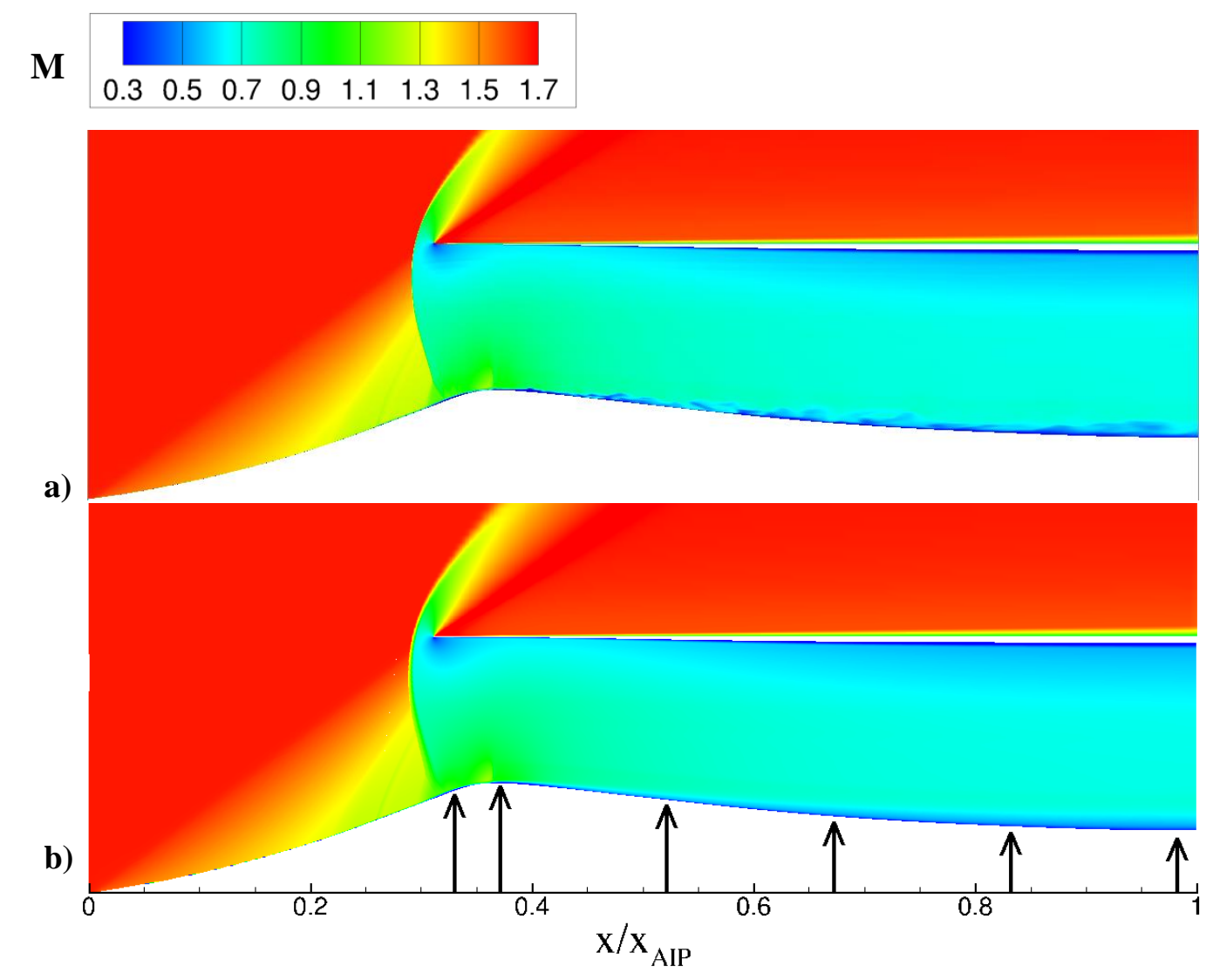

Fig. 2.4: DES-long fine Mach contours taken at the TDC plane: a) instantaneous and b) timeaveraged results with arrows indicating experimental Kulite tap locations at $x / x_{A I P}=0.33,0.37$, $0.52,0.67,0.83$, and 0.98 . 

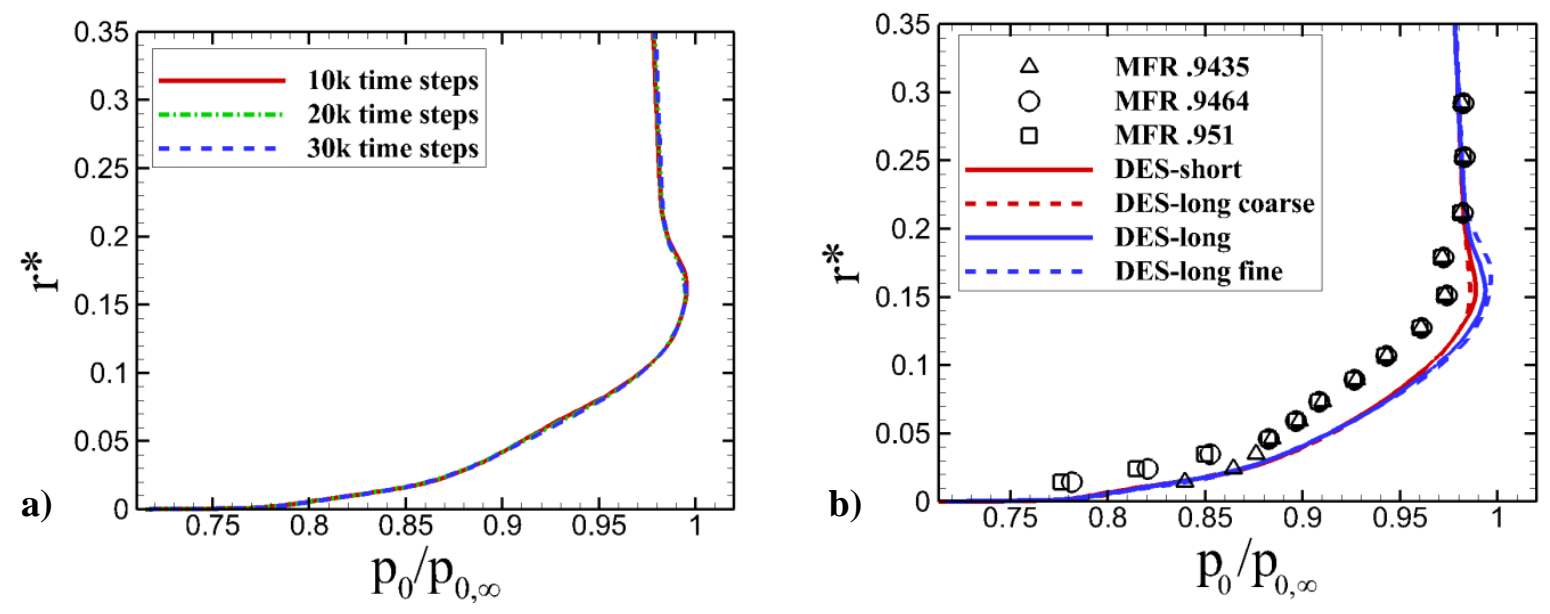

Fig. 2.5: AIP rake profiles showing stagnation pressure comparisons for a) temporal convergence of DES-long fine with profiles at three separate times, where $10 \mathrm{~K}$ time steps correspond to 0.01 seconds, and b) experimental, DES-short, and all three DES-long grid refinement levels. 

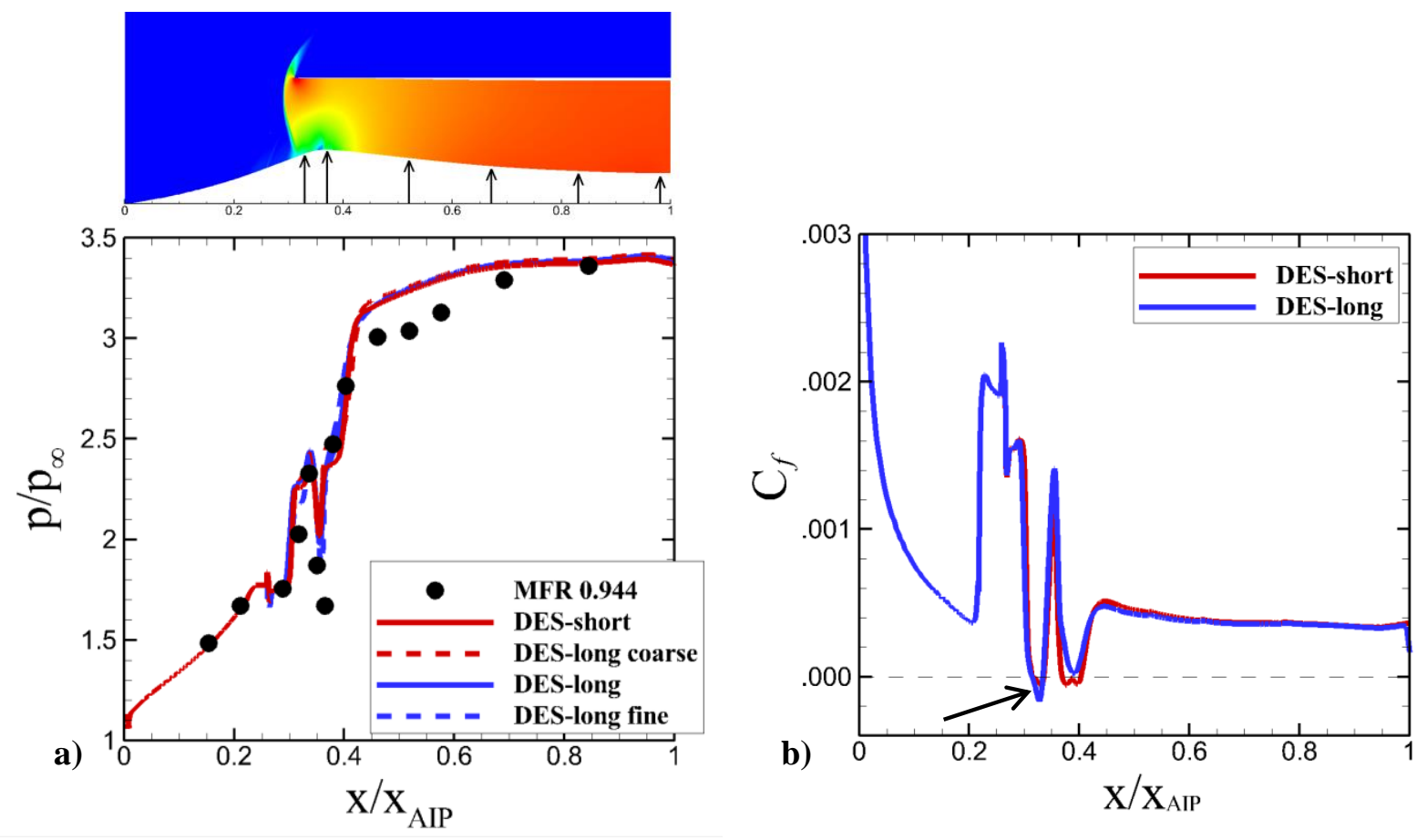

Fig. 2.6: a) Comparison of normalized average surface pressure distribution along inlet centerbody for both DES domain lengths, and experimental static pressure data. A contour of mean static pressure is seen above to reference the locations of Kulite pressure tap locations. b) Skin friction coefficient streamwise distributions along the centerbody for the DES-short and DES-long baseline resolutions. Negative regions indicated by an arrow represent separated regions. 


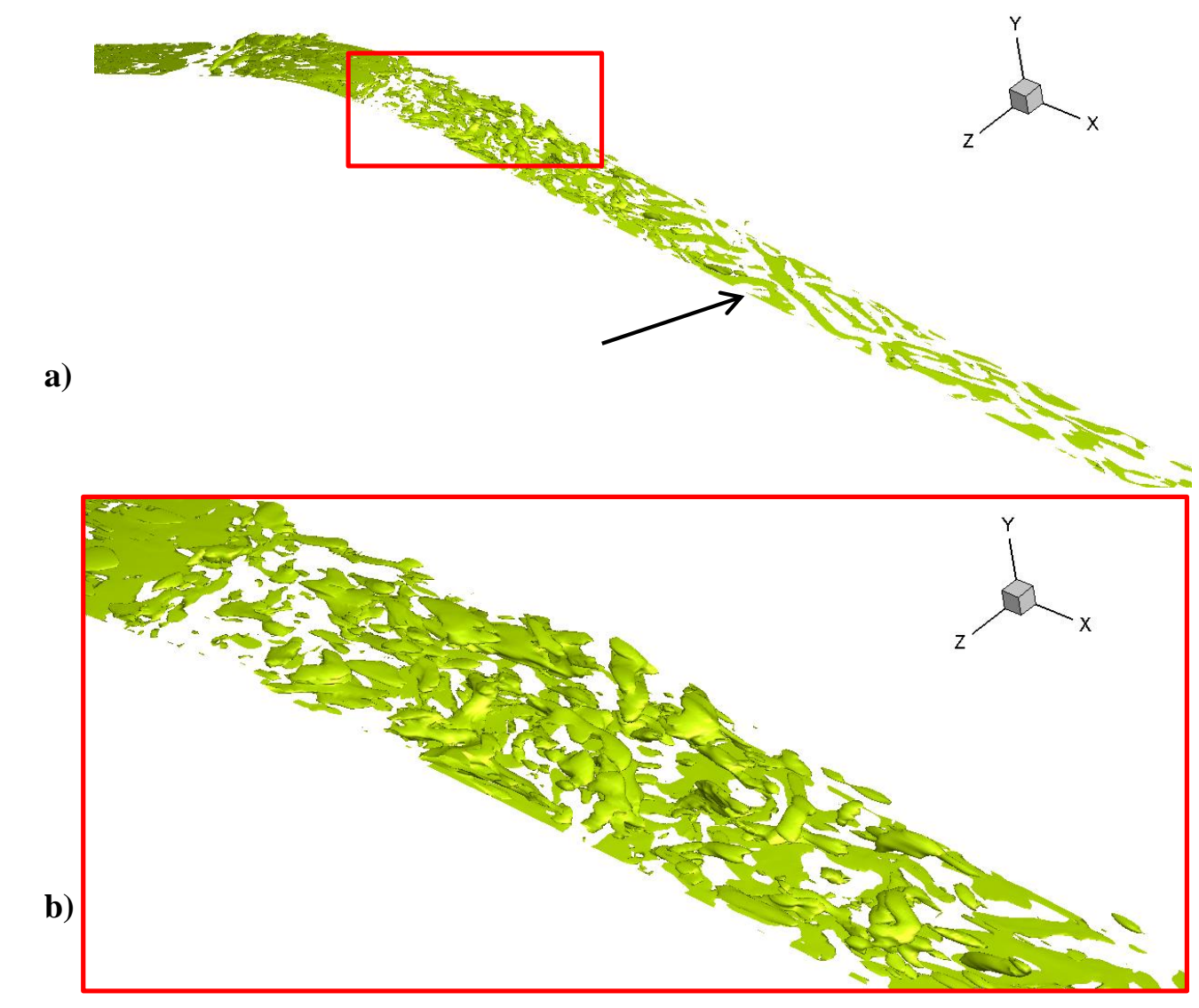

Fig. 2.7: Iso-surfaces illustrating turbulent structures in the near-wall region just downstream of the geometric throat using the Lambda-2 criterion from the DES-long fine results: a) overview from $x / x_{A I P}=0.27$ to $x / x_{A I P}=0.6$, and b) close-up region from $x / x_{A I P}=0.35$ to $x / x_{A I P}=0.46$. 

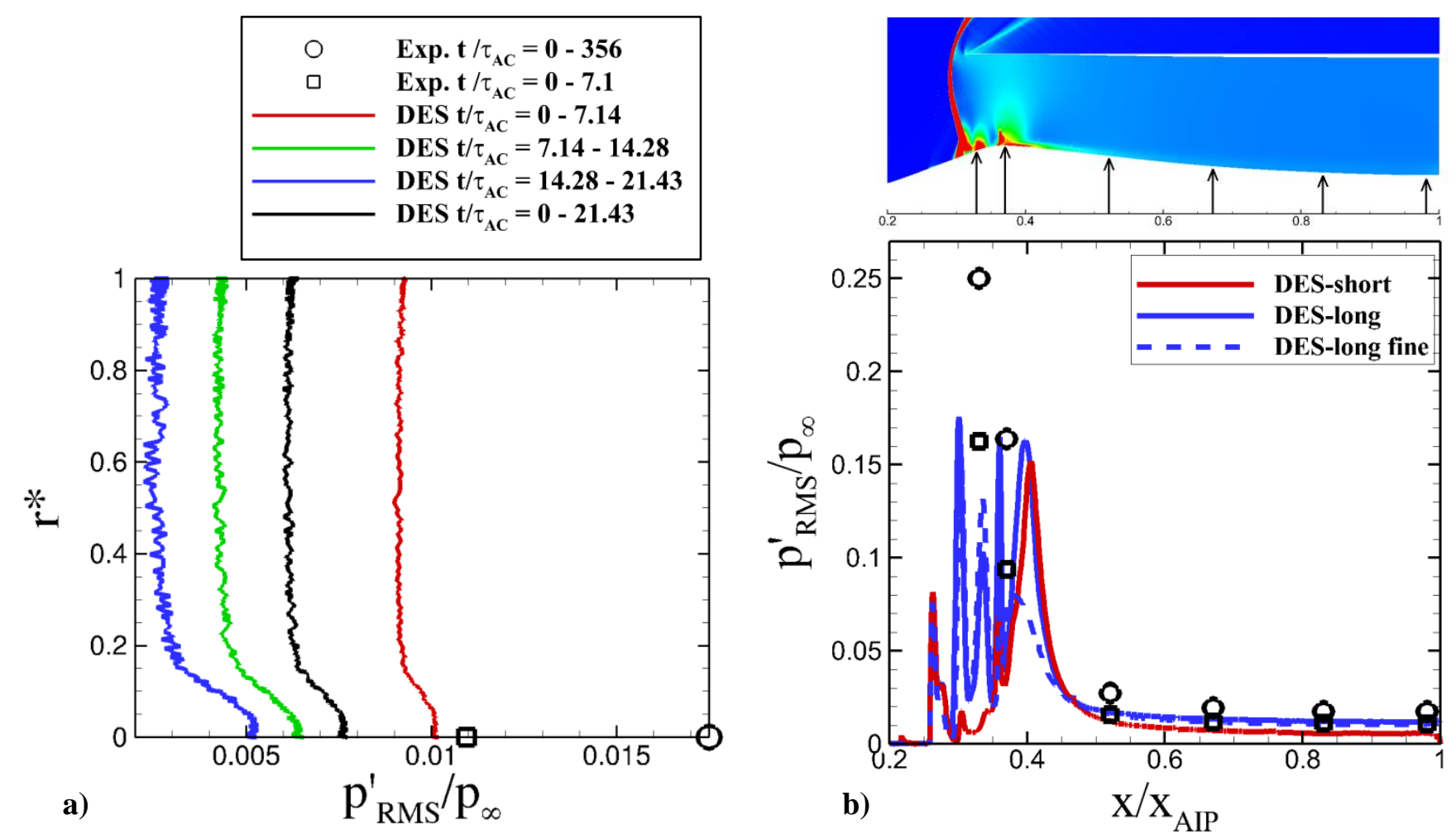

Fig. 2.8: Normalized $p^{\prime}{ }_{R M S}$ a) rake at the AIP showing three separate DES-long fine timeaveraged data sets (red, green, blue) and their resultant average (black), as well as b) streamwise surface distribution along the centerbody TDC slice for both DES-short and -long domains for integration times of up to $7.14 \tau_{A C}$ and experimental Kulite data. A DES $p^{\prime}{ }_{R M S}$ contour is seen above with Kulite tap locations. 

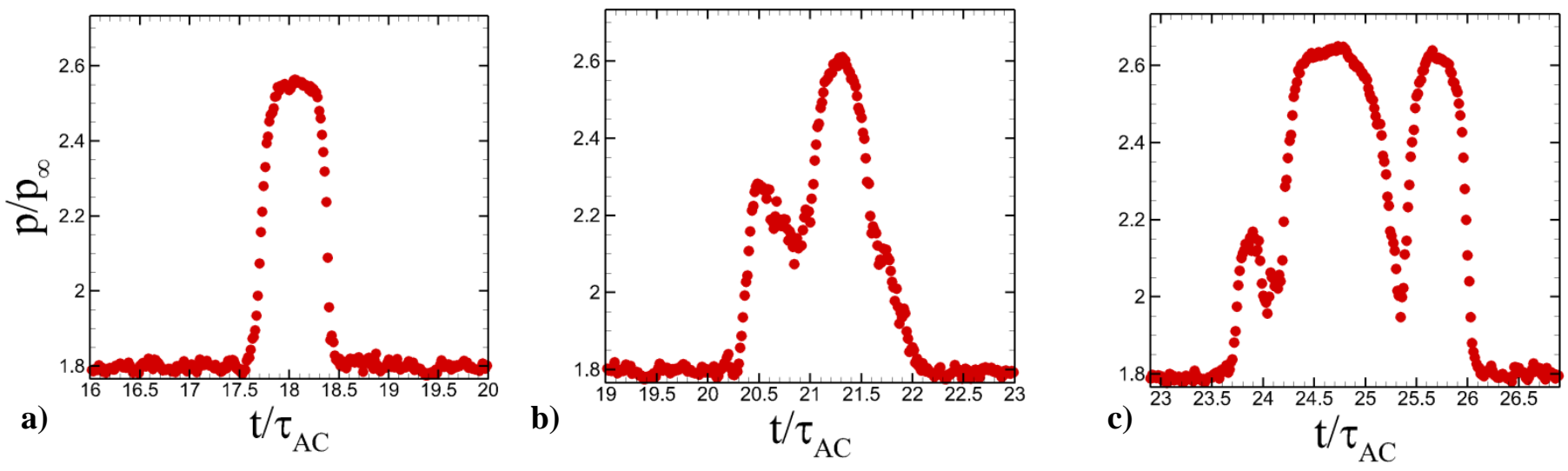

Fig. 2.9: Experimental pressure events occurring at a MFR of 0.96 and a streamwise location of $x / x_{A I P}=0.33$ for $\left.: \mathbf{a}\right)$ single-peak, b) double-peak, and c) triple-peak. 

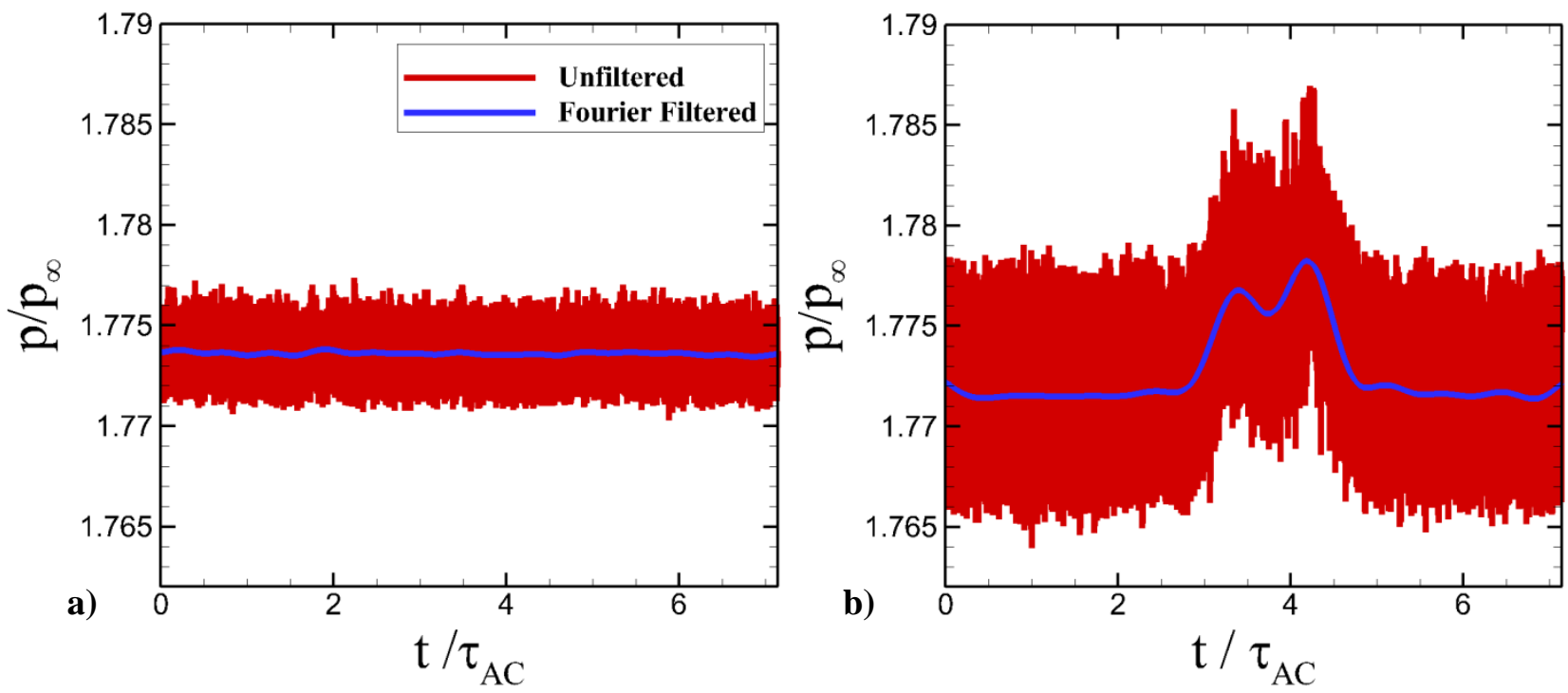

Fig. 2.10: Centerbody surface pressure trace at $x / x_{A I P}=.29$ location, just upstream of the normal shock, showing unfiltered and Fourier series filtered results using $f_{\text {cut }}^{*}=0.001$ for both a) DES-short and b) DES-long fine. 

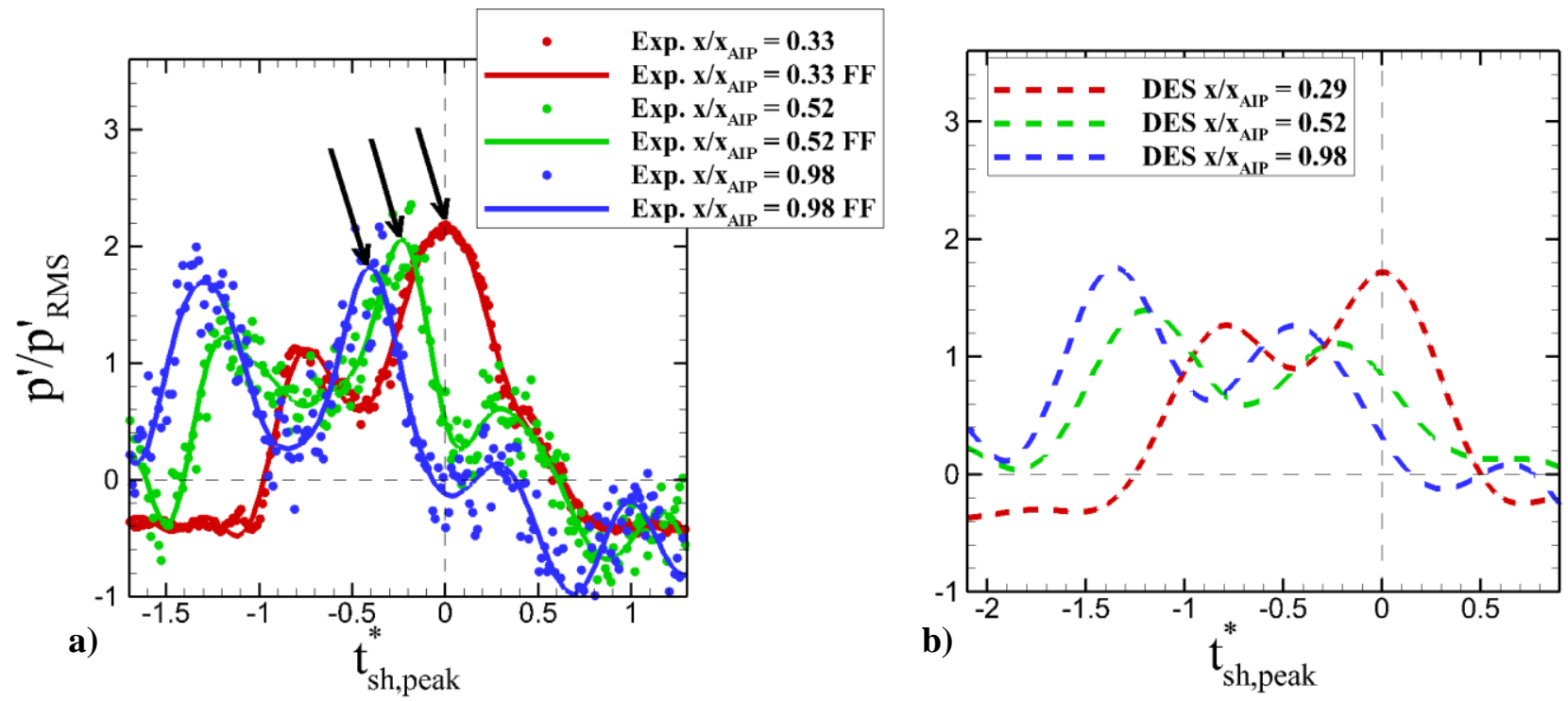

Fig. 2.11: a) Experimental double peak event at taps $x / x_{A I P}=0.33,0.52$, and 0.98 with both raw data points as well as $f_{\text {cut }}^{*}=0.01$ Fourier filtered (FF) curves and b) Fourier filtered DES results using $f_{\text {cut }}^{*}=0.001$ at $x / x_{A I P}=0.29$ and $f_{\text {cut }}^{*}=0.03$ at the remaining locations. The time origins are set to the occurrence of the pressure event at the respective upstream-most tap. 

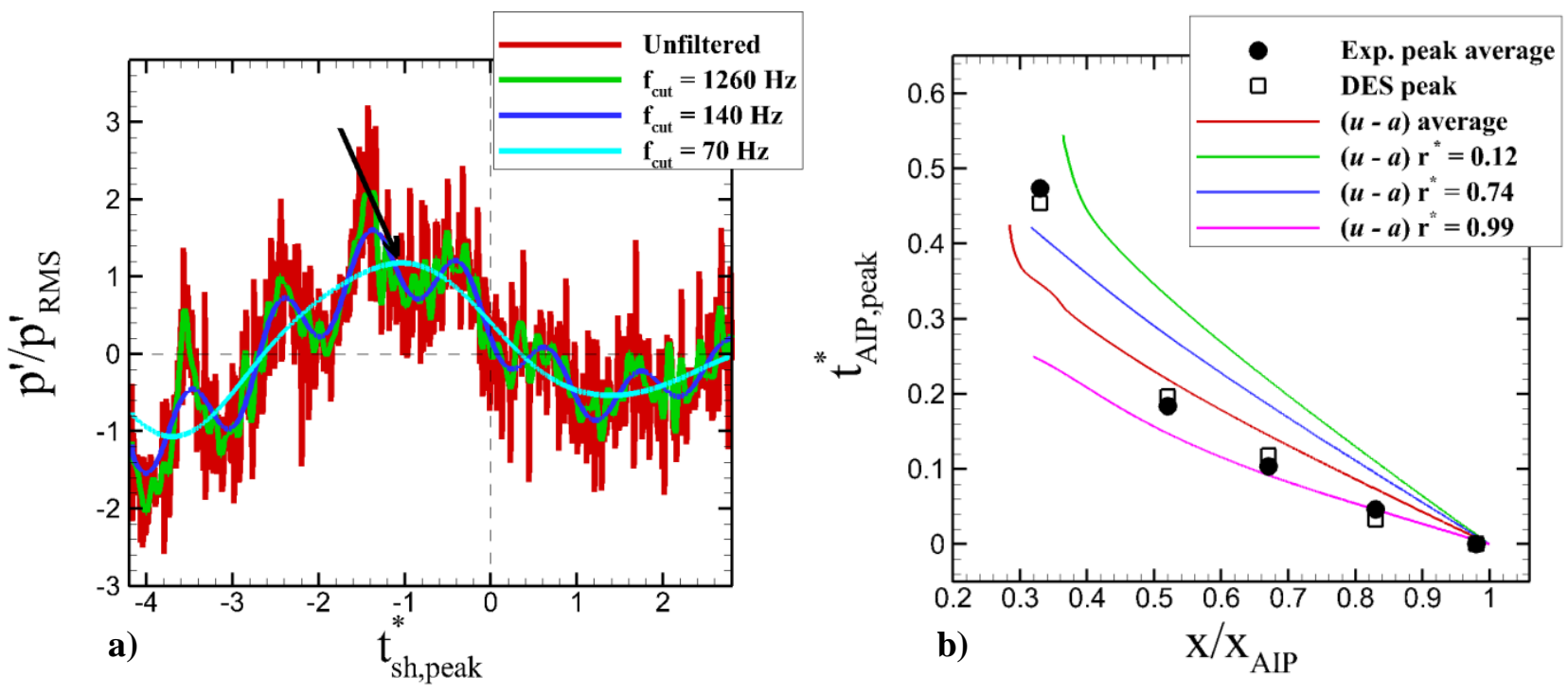

Fig. 2.12: a) Example of differing Fourier filter cut-off frequencies to represent the low frequency event at $x / x_{A I P}=.98$ of DES-long fine with a black arrow indicating where DES peak event times are taken, and b) time versus streamwise position of pressure event peaks and DES acoustic velocities at various wall normal distances and their spatial average. 
Table 2.1 Zonal start location and grid resolution for the Coarse, Baseline and Fine DES-long grids. The resolution for each zone is given in terms of the node indices for the $\mathrm{x}, \mathrm{r}$, and $\theta$ spatial directions as $i \times j \times k$. For the baseline resolution case, the blue highlighted text represents the zones used in the DES-short solution, whereas the baseline long domain used these points as well as those in Zones 16-20.

\begin{tabular}{|c|c|c|c|c|}
\hline Zone & $\begin{array}{c}\boldsymbol{x}_{\text {start }} \\
\boldsymbol{|} \boldsymbol{x}_{\text {AIP }}\end{array}$ & Coarse & $\begin{array}{c}\text { Baseline } \\
\text { (Short) }\end{array}$ & Fine \\
\hline 1 & -0.319 & $75 \times 100 \times 28$ & $75 \times 100 \times 32$ & $75 \times 100 \times 38$ \\
\hline 2 & 0.0 & $175 \times 100 \times 28$ & $175 \times 100 \times 32$ & $175 \times 100 \times 38$ \\
\hline 3 & 0.311 & $220 \times 100 \times 28$ & $220 \times 100 \times 32$ & $220 \times 100 \times 38$ \\
\hline 4 & -0.319 & $75 \times 270 \times 28$ & $75 \times 300 \times 32$ & $75 \times 330 \times 38$ \\
\hline 5 & 0.0 & $80 \times 270 \times 28$ & $80 \times 300 \times 32$ & $80 \times 330 \times 38$ \\
\hline 6 & 0.217 & $40 \times 270 \times 28$ & $40 \times 300 \times 32$ & $40 \times 330 \times 38$ \\
\hline 7 & 0.259 & $192 \times 270 \times 14$ & $212 \times 300 \times 32$ & $246 \times 330 \times 19$ \\
\hline 8 & 0.259 & $192 \times 270 \times 15$ & & $246 \times 330 \times 20$ \\
\hline 9 & 0.368 & $100 \times 270 \times 28$ & $250 \times 300 \times 32$ & $140 \times 330 \times 38$ \\
\hline 10 & 0.418 & $101 \times 270 \times 28$ & & $141 \times 330 \times 38$ \\
\hline 11 & 0.468 & $92 \times 270 \times 28$ & $100 \times 300 \times 32$ & $115 \times 330 \times 38$ \\
\hline 12 & 0.526 & $92 \times 270 \times 28$ & $101 \times 300 \times 32$ & $115 \times 330 \times 38$ \\
\hline 13 & 0.619 & $92 \times 270 \times 28$ & $100 \times 300 \times 32$ & $115 \times 330 \times 38$ \\
\hline 14 & 0.766 & $92 \times 270 \times 28$ & $101 \times 300 \times 32$ & $115 \times 330 \times 38$ \\
\hline 15 & 1.0 & $25 \times 75 \times 8$ & $100 \times 300 \times 32$ & $25 \times 75 \times 8$ \\
\hline 16 & 1.340 & $19 \times 75 \times 8$ & $19 \times 75 \times 8$ & $19 \times 75 \times 8$ \\
\hline 17 & 1.804 & $13 \times 19 \times 8$ & $13 \times 19 \times 8$ & $13 \times 19 \times 8$ \\
\hline 18 & 2.383 & $35 \times 19 \times 8$ & $35 \times 19 \times 8$ & $35 \times 19 \times 8$ \\
\hline 19 & 4.020 & $13 \times 19 \times 8$ & $13 \times 19 \times 8$ & $13 \times 19 \times 8$ \\
\hline 20 & 4.458 & $16 \times 19 \times 8$ & $16 \times 19 \times 8$ & $16 \times 19 \times 8$ \\
\hline
\end{tabular}

Table 2.2 Time averaged mass flow ratios for each DES case

\begin{tabular}{|c|c|}
\hline Case & MFR \\
\hline DES-short & 0.9491 \\
\hline DES-long coarse & 0.9449 \\
\hline DES-long & 0.9444 \\
\hline DES-long fine & 0.946 \\
\hline
\end{tabular}




\section{Chapter 3: Acoustically Induced Normal Shock Oscillations}

\subsection{Introduction}

The position and behavior of shock waves play a critical role in supersonic inlet design and analysis. The streamwise location of the shock is typically closely related to the resultant mass flow rate leading into the compressor region of the engine and understanding that relationship helps to define the operating conditions of the inlet. A phenomena known as inlet buzz occurs when the mass flow rate drops below a critical limit resulting in streamwise oscillations of the normal shock. Under certain conditions these oscillations exhibit large amplitudes and can cause structural integrity concerns. Therefore, understanding the causes and effects of inlet buzz is essential. Two types of buzz cycles have been characterized by Fisher $e t$ $a l .{ }^{17}$ referred to as "big" buzz and "little" buzz. Little buzz appears after small reductions in mass flow rate from on-design conditions, where the terminal shock begins to exhibit small amplitude oscillations. Continued reduction in mass flow rate results in big buzz where much larger oscillations occur, while the range of mass flow rates between the two types often exhibited fairly stable normal shocks. Fisher et al. ${ }^{17}$ determined the onset of little buzz by ingestion of a shear layer produced by the intersection of a strong oblique shock and the normal shock to within the cowl lip (known as the Ferri criterion $^{18}$ ). Dailey ${ }^{19}$ showed a relationship between shock oscillation frequency and the $8^{\text {th }}$ harmonic of a closed-end pipe of equal length of the diffuser, first suggesting acoustic waves as shock oscillation drivers. Trapier et $a l{ }^{20}$ experimentally investigated both types of buzz using a rectangular external compression supersonic inlet, where they showed acoustic waves are associated with little buzz leading to external shock oscillations. Such events have previously been investigated in both experimental and computational

studies $^{3,20-25}$. Some experimental set ups included rotating shafts in the downstream region to 
force upstream running compression waves ${ }^{22,25}$ and computational studies have investigated the use of downstream fluctuating pressure boundary conditions to mimic these effects ${ }^{24}$.

Schlieren video results of the present inlet from the NASA Glenn Research Center 8' x 6' supersonic wind tunnel tests have shown that on-design conditions of 0.985 MFR (or 1.5\% spillage) exhibit a stable shock, while a slightly decreased MFR of 0.955 produces a substantially more unsteady shock. Hirt et al. ${ }^{6}$ show that for this particular inlet, (big) buzz cycling with high amplitude shock movements does not begin until a MFR less than 0.4, which suggests the smaller oscillations at the higher MFRs to be more closely related to little buzz as described by Fisher et al. ${ }^{17}$. Chapter 2 studied the effect of downstream diffuser boundary conditions on unsteady wave propagation in DES, first using a fixed back pressure condition and then additionally modelling the experimental configuration downstream to the mass flow plug to capture possible acoustic reflections. Only the extended domain captured significant shock oscillations, and that upstream-running acoustic waves are seen to be the cause. Experimental unsteady pressure measurements also showed similar pressure waves traveling at acoustic velocities and resulting in normal shock oscillations.

With the exception of Chapter 2, characterization of wave propagation throughout inlet diffusers is generally done using spectral analysis in the frequency domain. Much of the previous low-boom research has focused on external overpressure and steady-state internal performance characteristics. The absence of a strong oblique shock (due to the isentropic compression spike) is a product of the low-boom design which may cause new effects when compared to general external compression inlets. The current study aims to further investigate the unsteady experimental pressure traces to reveal more information on compression wave propagation in the diffuser and the relation to normal shock oscillations. DES is used to show details of the internal 
flow-field that the experiments are unable to capture. Chapter 2 showed how unsteady Kulite taps can be used to observe streamwise shock oscillations if a surface pressure tap is located just upstream of the average shock position. The strong coupling between streamwise normal shock location and MFR limits this method to MFRs in the near-design regime approximately equal to 0.955. Therefore, the same experimental data set used in Chapter 2 exhibiting a 0.96 MFR will be used, along with the identical DES numerical approach. A detailed understanding of the structure and mechanics of the observed pressure wave and the resultant effect on normal shock position is sought.

\subsection{Experimental Methodology}

The wind tunnel tests were conducted at the $8^{\prime} \times 6^{\prime}$ supersonic wind tunnel at NASA Glenn Research Center. The tunnel can produce Mach numbers of up to 2.0 and utilizes wall perforations to control boundary layer growth along the test section walls. A 12" diameter scale inlet model is mounted in the test section upstream of a cold pipe leading downstream to the mass flow plug used to control the MFR. The inlet model extends 26.05 " from centerbody spike tip to the AIP $\left(x_{A I P}=26.05\right.$ "), which is the streamwise dividing plane between flight designed inlet geometry (upstream of the AIP) and the experimental rigging. Static pressure taps are located along the centerbody surface along with 8 unsteady Kulite pressure taps with a $5 \mathrm{kHz}$ sampling rate collected by a Dewetron data acquisition system. Each unsteady pressure trace contains five seconds of data yielding 25,000 pressure data points. The test section is run at a Mach number of 1.67 and $31.9 \mathrm{kPa}$ and $217^{\circ} \mathrm{K}$ static pressure and temperature, respectively, and a $0^{\circ}$ angle of attack for all cases considered in this study. The corresponding diameter and AIP streamwise distance based Reynolds numbers are 5.4M and 12M. The primary emphasis for mass flow rates is the 0.96 MFR case due to its corresponding normal shock streamwise location. 
The effect of MFR on the capture of normal shock motion is addressed with detail in the following sections. A sectioned view of the inlet model is shown in Fig. 3.1. A more detailed description of the wind tunnel tests can be found in Hirt et al. ${ }^{6}$.

\subsection{DES Methodology}

Hybrid RANS/LES methods, also commonly referred to as DES methods, have become increasingly popular for solving high Reynolds number flows involving complex geometries. Other time accurate numerical methods include direct numerical simulation (DNS) and large eddy simulations (LES) which typically require significantly more computational resources than DES. With the absence of turbulence modeling, DNS provides the greatest accuracy by resolving all scales of turbulence down to the smallest grid size, requiring very fine grid cells and therefore high computational expense. This expense is reduced in LES by sub-grid turbulence modeling, permitting slightly larger grid cells; however, in many cases LES is still impractical to solve high Reynolds number flows around complex geometries such as the current inlet flow-field. Due to the dependence on the minimum grid cell length, DNS and LES solutions rely on isotropic grid cells. In separated flow regions, this is typically not an issue since the turbulence length scales are large and therefore so too can the grid cells. However, in regions of high gradients such as boundary layers, small cells are required to resolve the gradient which is often significant only in one direction, creating high resolution in the remaining two directions where it may not be required.

The DES method approximately reverts to a RANS method in regions of attached boundary layers where a high gradient exists in the wall normal direction. This allows the DES approach to use computationally efficient high aspect ratio (anisotropic) grid cells in these regions with high spatial resolution in the wall-normal direction (where spatial gradients are 
highest) while discretization in the streamwise and spanwise directions may remain coarser. This drastically reduces the total number of grid points relative to the requirements of a DNS or LES approach with the corresponding cell isotropy requirement. For separated flow regions, the DES method approximately reverts to a LES method with sub-grid turbulence modeling to solve for regions exhibiting large-scale turbulence. The cell isotropy condition still applies for the LES regions, but since these occur in separated flow regions the turbulent length scales will be large and therefore so too can the grid cells. This significantly reduces the number of grid cells required for DES when compared to LES or DNS when generating time accurate flow simulations. Additional details can be found in Rybalko and Loth ${ }^{11}$, Rybalko et al. ${ }^{7}$, and Chapter 1 further justifying the use of DES for the current inlet flow.

The Nichols-Nelson hybrid RANS/LES methodology ${ }^{13}$ is chosen for the present study since it employs both grid and turbulent length scales for its blending function, allowing smooth transition between RANS and LES while reducing the possibility of grid induced separation, which may be a concern with traditional DES methods. The upper region boundary layer is most commonly susceptible to grid induced separation where wall distance may surpass the grid length scale. This causes an LES solution to be used in the local region which results in a reduction of modeled eddy viscosity. LES typically compensates for this reduction by instead resolving the turbulence down to the local grid scale. However, anisotropic grid cells that exist in the upper region of the anticipated boundary layer do not provide adequate resolution for an LES solution. This under prediction of turbulence often leads to numerical separation causing potentially significant errors in predicted flow properties ${ }^{15}$. The Nichols-Nelson approach was constructed around the Menter SST turbulence model, which is employed throughout the computational domain. 
The DES results presented in the current study use a $16.7 \mathrm{M}$ point grid, which is equal to the fine resolution case of Chapter 2 that showed sufficient spatial resolution for all timeaveraged features. The computational flow domain is divided into 20 separate zones for parallel computation and solves from upstream of the centerbody spike tip downstream to the mass flow plug. A $3^{\text {rd }}$ order upwind biased Roe_over scheme (an implementation from the OVERFLOW code) is used for spatial discretization, and the $2^{\text {nd }}$ order approximate factorization alternating direction implicit (AF ADI) method is used for time integration. The Superbee TVD limiter is used to preserve monotonicity across shock waves. Periodic boundary conditions are used at spanwise boundaries, and the outflow boundary plane near the mass flow plug is set to a constant, near-zero pressure. The geometric size of this outflow region is adjusted until the desired MFR is reached. A constant time-step of 1e-6 seconds is used and 0.1 seconds of timeaveraged data is gathered once the flow has been initialized and transients due to initialization have dispersed. TDC slices are output every 20 time-steps for high temporal resolution video generation. Detailed descriptions of the DES method and solution strategies as well as grid resolution studies can be found in Rybalko and Loth $^{11}$, Rybalko et al. ${ }^{7}$, as well as Chapter 2.

\subsection{Shock Oscillation Capture Approach}

Experimentally capturing a shock oscillation using only an unsteady surface pressure signal requires particular positioning of the experimental tap and the normal shock. Figure 3.2 illustrates the challenges faced when attempting this. Examples of the observed pressure traces for several MFRs are presented in Fig. 3.2a with approximate locations of the corresponding normal shocks in Fig. 3.2b. If the normal shock is located too far downstream of the tap (0.985 MFR, blue curve), only relatively stable supersonic pressures will be observed. Too far upstream of the tap (0.9 MFR, green curve) and only subsonic pressure will be recorded. An average 
normal shock position that is just downstream of the Kulite tap (0.96 MFR, red curve) is required in that it produces high amplitude pressure peaks as the shock moves across the tap (see Fig. 3.2a). These pressure peaks allow for an indirect capture of normal shock oscillations at a neardesign MFR of 0.96 due to the unique positioning of the shock and the Kulite tap in relation to one another. Also indicated in Fig. 3.2b are black arrows that represent streamwise position of the six experimental Kulite taps.

Chapter 2 illustrated the presence of multiple shock waves in the current inlet flow-field, which will be briefly reviewed. Figure 3.3 shows DES Mach contours at the top dead center (TDC) plane that highlight the normal shock location at a 0.94 MFR. Figure 3.3b shows a close up of the normal shock and the centerbody throat region with an adjusted contour scale. The normal shock is clearly illustrated, as is a second shock located near the throat. With the introduction of a second shock wave, the upstream terminal shock will continue to be referred to as the "normal shock" while the second shock will be labeled the "throat shock." Preceding the throat shock is a region of expansion due to flow turning which accelerates the flow to supersonic velocities. This expansion region will be referred to as the "throat expansion." The referencing to each structure can be found in Fig. 3.3b. Again, black arrows indicate the experimental Kulite tap locations with $x / x_{A I P}=0.33$ being the upstream-most tap in Fig. 3.3b. A DES tap placed at the same location would not have captured the shock oscillation at the DES 0.94 MFR because the normal shock was located too far upstream, and therefore pressure was extracted at every time-step at $x / x_{A I P}=0.29$ indicated with a red arrow. The increased 0.96 MFR of the experiments will exhibit a shock with average location farther downstream than the DES. 
To best compare DES to experiments, identical computational MFRs are sought. The MFR is controlled in the DES by altering the size of the outflow plane. This involved defining an outflow area and solving the flow to calculate a time-averaged MFR. Based on the resultant average, the outflow area is adjusted and the process repeats. This iterative method was quite time consuming and therefore small MFR differences were deemed acceptable.

The DES trace at $x / x_{A I P}=0.29$ was placed farther upstream of the average normal shock position compared with the experimental upstream tap, reducing the effect of normal shock oscillations at the DES tap. This results in the shock oscillation being masked by high frequency turbulence, therefore requiring a low-pass filter to reveal the low frequency shock oscillation. This is done by decomposing the DES pressure signal into a Fourier series which clearly illustrates normal shock motion. Examples of the Fourier series filter and comparisons to unfiltered data can be found in Chapter 2

Figure 3.4 shows the experimental pressure traces at all six streamwise locations for the 0.96 MFR case, which will be the only experimental case considered from this point. Large spikes in pressure illustrate shock oscillations at the upstream-most tap in Fig. 3.4a. These oscillations occur as single-peak and multi-peak events. The single-peak events can be most easily characterized because of their Gaussian distributions and are herein the only types considered. To quantify the time of each of these oscillations, an shock oscillation "event" was defined in terms of pressure recording exceeding the threshold value of $p / p_{\infty}=2.0$. When such an event is identified to have occurred, the pressure recording was investigated to determine the time when this threshold occurs with a pressure rise (before the peak time) and when this threshold occurs with a pressure drop (after the peak time). These two threshold crossing times were then averaged to define the pressure peak time $t_{\text {sh,peak }}$, which is expected to be consistent 
with the time at which the shock has moved farthest upstream By defining this event time, the

remaining Kulite taps at other stations can be viewed in reference to this to better understand how the events are correlated in space and time. Six different shock oscillations, or "pressure events," at the upstream most tap are identified, averaged, and examined in detail at all locations. Time is normalized by an acoustic time scale $\tau_{A C}$ which is defined as the time required for an acoustic wave to propagate from the geometric throat to the mass flow plug and reflect back upstream to the throat. It was calculated using DES mean flow and sound speeds.

\subsection{Pressure Event Description}

To understand what kind of streamwise waves are traveling throughout the diffuser, the pressure traces must be examined at each location both over long and short time spans. Long time evolution of the pressure signal is seen in Fig. 3.4 for all six streamwise tap locations. Normalized time extends from $t / \tau_{A C}=0$ to $t / \tau_{A C}=75$, which is only $20 \%$ of the five-second experimental trace. The upstream most tap in Fig. 3.4a, located at $x / x_{A I P}=0.33$, illustrates the shock oscillating over the tap by generating pressure spikes as the shock moves upstream and the tap becomes exposed to high pressure subsonic flow. Figure $3.4 \mathrm{~b}$ shows $x / x_{A I P}=0.37$ where similarly sized amplitude oscillations are occurring but at a higher frequency than the upstream tap. Chapter 2 illustrated the presence of a second normal shock in the vicinity of the geometric throat using DES, which is also seen in Fig. 3.3b by means of Mach contours. The comparable amplitude of data from the two upstream experimental taps suggests that a common mechanism causes these oscillations, indicating the presence of a shock near the throat at $x / x_{A I P}=0.37$. Experimentally observing these high amplitude oscillations also indicates that the near-throat shock exhibits an unsteady streamwise position, elsewise these fluctuations would not be captured. The remaining taps located in the subsonic diffuser region at distances of $x / x_{A I P}=$ 
0.52, $0.67,0.83$ and 0.98 begin to show a low frequency saw-tooth like wave motion in Figs. 3.4c-f. High frequency, low amplitude waves are also seen superimposed on the low frequency saw-tooth motion, likely caused by the turbulent nature of the diffuser boundary layer.

To understand the structure of one of these waves, these events were analyzed with higher temporal resolution and with consistent event reference frames. Figure 3.5 shows pressure traces over a dimensionless time span of 3 for six separate pressure events at the upstream tap at $x / x_{A I P}=0.33$. The average of the six curves is shown in black in all images. A new time scale is introduced which adjusts the origin to be equal to the peak upstream normal shock position at $t_{\text {sh,peak }}$ and is defined as

$$
t_{\text {sh,peak }}^{*}=\left(t-t_{\text {sh,peak }}\right) / \tau_{A C}
$$

The peak of the averaged curve occurs approximately at $t_{s h \text {,peak }}^{*}=0$ illustrating the Gaussian shape of each pressure wave. Between $t_{s h, p e a k}^{*}=-1.5$ and $t_{s h, p e a k}^{*}=-0.5$ the normalized pressure is fairly stable in all events with the exception of event 4 in Fig. 3.5d. Event 4 experiences lesser amplitude oscillations just prior and just after the event, likely due to similar waves that result in a shorter upstream shock motion. The stable $p / p_{\infty}=1.8$ at the beginning of each trace exemplifies supersonic flow where only minor pressure fluctuations are seen due to boundary layer turbulence. The start time of a shock oscillation is defined as where the pressure initially begins to increase and is referred to as $t_{s h \text {,start }}^{*}$ Similarly, the end time of a shock oscillation is defined as where the pressure returns to stable supersonic pressure after the oscillation, labeled $t_{s h, e n d}^{*}$. The averaged distribution in Fig. 3.5 shows a start time just greater than -0.5 and an end time just less than 0.5 . The greatest peak pressure of $p / p_{\infty}$ of 2.56 occurs during event 1 which also experiences the greatest $\left(t_{s h \text {,end }}-t_{s h, s t a r t}\right) / \tau_{A C}$ of 0.957 . Additional details of each event can be found in Table 3.1. The frequency of these oscillations varies for the whole trace, with 
many occurring in series shortly after one another (see Fig. 3.4d) or even occurring on top of each other forming double- or triple-peak events.

Moving to the midstream tap located at $x / x_{A I P}=0.52$, Fig. 3.6 shows each of the selected six pressure events and their averaged distribution in the identical time frame as Fig. 3.5. The pressure trace is more complex at the downstream regions than it is at the upstream tap in Fig. 3.5 due to increased wave propagation dimensionality, possible flow separation and a more turbulent diffuser boundary layer. Here, the pressure trace is composed of two apparent peaks, the initial peak being of greater amplitude than the second, with the $t_{s h \text {,peak }}^{*}=0$ vertical dashed line intersecting at about $70 \%$ of the way down the downslope of the first peak. When viewing events at this midstream location, one point of interest is the high pressure peaks. The first peak of the average curve occurs at approximately $t_{s h \text {,peak }}^{*}=-0.3$, illustrating that the downstream pressure peaks before the normal shock streamwise position does. The same trend is seen when observing the start time of when the pressure begins to increase. This indicates an upstream running wave if in fact the pressure peaks at the midstream tap in Fig. 3.6 are associated with the normal shock motion in Fig. 3.5. Figure 3.7 continues to the downstream most tap, located just ahead of the AIP at $x / x_{A I P}=0.98$. The pressure traces look very similar in shape compared with the midstream tap seen in Fig. 3.6, however, the pressure is generally greater due to the increasing cross sectional area of the diffuser. The time phase shift is more pronounced here than at the midstream, with the average local maximum occurring just after $t_{s h \text {,peak }}^{*}=-0.5$. It is clear that the downstream tap is the first to experience the onset of the pressure increase and pressure peak, followed in time by the midstream and finally the upstream-most tap, continuing to indicate the presence of an upstream running compression wave. 


\subsection{Wave Propagation Velocity}

Types of streamwise waves capable of propagating upstream in a subsonic diffuser include shock waves, expansion fans, and acoustic waves. Rybalko et al. ${ }^{27}$ show that for some flow-fields shock oscillation frequency may depend on the characteristic length scale of the separation bubble produced downstream of a normal SWBLI. A similar SWBLI and resultant separated region is observed in the current flow-field, implying that unsteadiness of the separated region may also be a source of normal shock motion. However, Rybalko ${ }^{2}$ illustrates that acoustic frequencies are more likely to be the cause in the present flow-field based on frequency analysis of schlieren video. The presently observed wave cannot be a shock wave since DES Mach contours fail to show any indication of significant discontinuities throughout the diffuser in all regions with the exception of the geometric throat. An upstream running expansion fan would cause decreases in pressure, the opposite of what is observed by the experimental Kulite taps, leaving an acoustic wave as the probable wave type. A detailed investigation of the time phase shift will help understand the velocity of the upstream running wave to determine if it travels at an acoustic velocity.

Figures 3.5-3.7 clearly show upstream propagation of a compression wave with a resultant normal shock upstream motion. To better illustrate the time phase shift between pressure events at various streamwise locations, Fig. 3.8 shows a comparison between pressure fluctuations at the upstream, midstream, and downstream taps, located at $x / x_{A I P}=0.33,0.52$, and 0.98 , respectively. Figure $3.8 \mathrm{a}$ shows normalized pressure fluctuations of the three taps, which clearly show the effect of the shock oscillation on surface pressure fluctuations. The

upstream tap, shown in red, exhibits a significantly stronger fluctuation around $t_{\text {sh,peak }}^{*}=0$ due to the brief exposure of high, post-shock pressure during shock motion. The midstream and 
downstream taps show fluctuations which are approximately of equal amplitude to one another, but far weaker than the upstream. Due to the differences in magnitude, the fluctuations are again normalized by their respective root mean square (RMS) values. This normalization creates curves of equal magnitude so that the time phase shift between streamwise locations is clearly illustrated in Fig. 3.8b. A simplified pressure limit is calculated using the theoretical onedimensional shock relations across an oblique and normal shock in series to replicate the lambda structure at the base of the normal shock (see Fig. 3.3b). Time-averaged Mach numbers are extracted from the DES upstream of the oblique shock, between the oblique and normal shocks, and downstream of the normal shock, and are then used to calculate the theoretical pressure increase after passing through the lambda structure. The experimental time-averaged pressure at the upstream tap is subtracted from the calculated theoretical pressure to convert to a fluctuating value, plotted in Fig. 3.8a. This simplified theoretical representation of the lambda shock structure is compared to the peak pressure at the upstream tap, where the theoretical prediction exceeds the measured value. This discrepancy is likely due to viscous effects in the boundary layer which are neglected in the one-dimensional calculation.

Characterizing the time of the event $\left(t_{s h, p e a k}\right)$ for the upstream case was trivial due to its simple symmetric shape. Characterization of the event time at the midstream and downstream taps is more complex. Key moments of interest of each trace are recorded. Both the blue and green curves indicate an incoming $p^{\prime} / p^{\prime}{ }_{R M S}$ of approximately zero in Fig. 3.8b, indicating average pressure. The pressure then exhibits two periods of sinusoidal-like oscillations. The initial steep increase is representative of the moment that the high pressure wave crosses the Kulite tap. The beginning of this increase is labeled as the "start," of the pressure event, and is indicated by arrows in Fig. 3.8b. Another point of interest is the local "peak" time, also indicated 
by arrows. Both the start and peak times of each individual pressure event are recorded at all streamwise locations, with the exception of the tap at $x / x_{A I P}=0.37$ where the pressure trace exhibits far more complex events due to the presence of the throat shock. The events of this tap will be addressed in detail in the following section. The recorded start and peak times from Figs. 3.5-3.7 will determine the time phase shifts between taps. The phase shift can be used to track wave propagation using the known streamwise locations of the Kulite taps.

Acoustic waves have been shown in the past ${ }^{2,20-25}$ to reflect from downstream geometries and propagate upstream to cause normal shock unsteadiness. Streamwise distributions of the time-averaged DES predicted upstream acoustic propagation velocity, defined as $(u-a)$, is extracted at various wall-normal heights in the diffuser. Using this propagation velocity, the predicted change in position can be calculated across each grid cell to obtain the streamwise displacement curve of an acoustic wave in time. These curves are extracted at normalized wallnormal heights using

$$
r^{*}=\left(r\left(x_{A I P}\right)-r_{C B}\right) /\left(r_{c o w l}-r_{C B}\right)
$$

This radial measure is defined as the height off the centerbody normalized by the clearance between the centerbody and the cowl inner surface. The heights of $r^{*}=0.12,0.74$ and 0.99 are chosen as they exemplify the entire range of acoustic velocities based on off-wall height, and a spatial-average is calculated between $r^{*}=0$ to 1 . Additionally, two new time scales are introduced and defined as

$$
\begin{aligned}
& t_{A I P, \text { start }}^{*}=\left(t-t_{A I P, \text { start }}\right) / \tau_{A C} \\
& t_{A I P, \text { peak }}^{*}=\left(t-t_{A I P, \text { peak }}\right) / \tau_{A C}
\end{aligned}
$$


The onset of the pressure rise and the local pressure maximum at the near-AIP tap $\left(x / x_{A I P}=\right.$ $0.98)$ are denoted as $t_{A I P, s t a r t}$ and $t_{A I P, p e a k}$, respectively, and are indicated with arrows in Fig. 3.8b. These time scales shift the time origin to the start and peak times of the near-AIP tap resulting in positive time values for upstream running waves at the remaining taps. Streamwise position versus dimensionless time is plotted in Fig. 3.9 for experimental pressure events and for a DES acoustic wave. Figure 3.9a uses the experimental start times $\left(t_{A I P, s t a r t}^{*}\right)$ as symbols for the six recorded pressure events along with their average. The four downstream taps located in the diffuser show relatively good agreement with the DES predicted acoustic wave propagation with the majority of the events falling within the range of DES velocities. Event 1, seen as red squares, has a shallower slope than the remaining events indicating a faster upstream propagation velocity. The peak pressure values at the near-AIP tap from Fig. 3.7 are approximately equal for all events, however, event 1 produces the greatest peak pressure and the greatest $\left(t_{\text {sh,end }}-\right.$ $\left.t_{s h, s t a r t}\right) / \tau_{A C}$ at the upstream tap (see Table 3.1) suggesting normal shock displacement is more sensitive to wave velocity than it is to compression strength. The tap at $x / x_{A I P}=0.33$ does not show good agreement in Fig. 3.9a likely caused by the transonic region around the throat shock. Extracting the event start time proved challenging for certain events at the downstream taps. Figure 3.7d shows event 4 with a clear start time where a local pressure minimum exists at $t_{s h, p e a k}^{*}=-1$. The start time for event 2 and 5 seen in Figs. 3.7b and 3.7e do not exhibit clear local minimums to extract the start time. This variability must be considered when evaluating the propagation distribution based on the start times in Fig. 3.9a. Extracting the pressure wave peak times was far more consistent since each event exhibits clear local maximums. The wave propagation based on the peak times $\left(t_{A I P, \text { peak }}^{*}\right)$ is shown in Fig. 3.9b. The DES acoustic propagation curves show the shallowest curve at $r^{*}=0.99$, just below the inner cowl surface 
where the flow is far away from the transonic region at the geometric throat. Moving towards the centerbody, the $r^{*}=0.74$ curve shows a steeper curve and therefore a slower upstream acoustic velocity, and the slowest presented acoustic velocity is predicted at $r^{*}=0.12$ near the centerbody surface. Each of these curves end when the flow becomes supersonic and acoustic waves can no longer continue upstream. The experimental peak times show reasonable agreement of the four diffuser taps to the red spatially-averaged DES curve in Fig. 3.9b. Most points fall within the range of acoustic propagation curves with the greatest deviation from the spatial-average at the upstream-most tap. The wave front of an acoustic compression wave will be three dimensional due to the three dimensionality of the velocity throughout the diffuser, making the distributions seen in Figs. 3.9a and 3.9b simplified versions of a complex propagation front. However, the agreement seen between the DES acoustic propagation and the experimental propagation of both the pressure wave start and peak times provides evidence that the compression waves are indeed traveling at acoustic velocities.

The large scale motion of the compression wave can be observed using DES results to understand how it affects the normal and throat shocks. Several TDC plane contour plots of normalized pressure fluctuations are shown in Fig. 3.10 to illustrate the upstream propagation in the DES. Seven frames are shown at various times during the passage of a single wave indicated on the right by the Fourier filtered pressure trace from the upstream $x / x_{A I P}=0.29$ DES tap. Figure 3.10a begins at $t_{s h \text {,peak }}^{*}=-2.03$, while the pressure wave is downstream of the AIP and where the overall pressure fluctuations are near zero with the exception of the throat region. The normal shock is illustrated by a band of blue and red contours of equal streamwise thickness, indicating average streamwise shock position. Strong pressure fluctuations are seen at the geometric throat caused by the throat shock as well as flow separation downstream of the normal 
shock. The expansion region can be seen with low pressure contours just ahead of the throat shock. Figure $3.10 \mathrm{~b}$ continues in time to just as the pressure trace indicates the normal shock is beginning to move upstream. At this point, the pressure wave appears to be just at the AIP based on the contour plots, however, the wave is not defined by a discontinuous front, like a shock would be, and therefore exhibits a gradual pressure increase. The gradually increasing pressure extends across a large streamwise length, continuing to move upstream at $t_{s h \text {,peak }}^{*}=-1.11$ in Fig. $3.10 \mathrm{c}$ pushing the normal shock upstream. Figures 3.10a and 3.10b show a series of high-to-low pressure fluctuation regions just downstream of the normal shock. These regions are indicative of boundary layer perturbations that increase or decrease the local pressure depending on the perturbation thickness and advect downstream. Figure 3.10d shows the low between the double peaks of the DES pressure trace while the length of the wave continues to propagate from the downstream region and impinge on the normal shock. The shock is at its upstream-most location in Fig. 3.10e and the tail end of the pressure wave is visible downstream of the throat. Figures 3.10f and $3.10 \mathrm{~g}$ illustrate the shock receding back to its average location and the pressure returning to average values. The diffuser region in Fig. $3.10 \mathrm{~g}$ exhibits blue contours, indicative of negative pressure fluctuations and therefore below average pressure. This is due to the increase in time-averaged pressure from the observed compression wave, and the blue negative pressure fluctuation contours actually correspond to stable normal shock conditions. These instantaneous snapshots of pressure fluctuations illustrate the upstream motion of a compression wave resulting in normal shock upstream movement. The full pressure fluctuation video can be found in Fig. 3.11. 


\subsection{Geometric Throat Shock Dynamics}

The experimental pressure trace at $x / x_{A I P}=0.37$ has not yet been addressed in detail due to the increased complexity at the throat region. Mach contours of the DES at the TDC plane have illustrated the existence of the throat expansion and throat shock in Fig. 3.3b. Previously, Fig. 3.4b has shown high amplitude pressure fluctuations comparable to those caused by the normal shock seen in Fig. 3.4a at the upstream tap, suggesting possible streamwise motion of the throat shock. Figure 3.12a shows a small time window around $t_{s h \text {,peak }}^{*}=0$ of all six experimental pressure events and their average. The individual events strongly differ from one another at most times except for the local minimum occurring after $t_{s h, p e a k}^{*}=0$. This disagreement between events indicates complex, somewhat random interactions are occurring in the near-throat region. The region of agreement between events begins at approximately $t_{s h \text {,peak }}^{*}=-0.4$ where a pressure decrease is observed. A local peak is seen in most of the events around $t_{s h \text {,peak }}^{*}=0.6$, where the events then diverge from one another. The agreement between events occurs over a range of $t_{\text {sh,peak }}^{*}=1$, slightly greater than the 0.623 average time of the shock motion from Table 3.1. The averaged curves of all six pressure events are shown in Fig. 3.12b for the experimental taps located at $x / x_{A I P}=0.33$ and $x / x_{A I P}=0.37$. It is clear from Fig. $3.12 \mathrm{~b}$ that the local minimum at the throat tap occurs after the upstream tap has peaked and the normal shock has reached its maximum upstream position. This, along with the fact that the pressure reaches a minimum and not a maximum at $x / x_{A I P}=0.37$, suggests that the motion of the normal shock, not the acoustic wave, drives the pressure minimum at the near-throat tap. This is also supported by the supersonic velocities in the throat expansion region which would prohibit an acoustic wave from propagating any farther upstream. 
To better understand the behavior of the throat shock, its interaction and response to the normal shock is investigated. Due to the streamwise motion of the normal shock, changes in normal shock strength may occur which would create changes in pressure and velocity leading into the throat shock. Instantaneous DES contours illustrate the dynamics of the throat expansion and shock. Turbulent eddies are shed from the normal SWBLI that cause high frequency perturbations in throat expansion region size and small streamwise oscillations of the throat shock. The high frequency of this shedding is likely the cause of the disagreement between events in Fig. 3.12a which also occurs at a high frequency. These eddies may entrain low pressure from the upstream supersonic region or high pressure from post-shock compression, which then advect downstream to the throat Kulite tap. Depending on the pressure contained in each eddy, high or low pressure will be observed by the experimental tap which may explain the large range of pressure magnitudes of the events in Fig. 3.12a.

The pressure minimum seen by all events in Fig. 3.12a occurs at a low frequency similar to that of the normal shock motion. The instantaneous DES data shows that as the normal shock moves, variations in throat expansion size and increased streamwise motion of the throat shock occurs. The streamwise locations of the normal shock and throat shock appear to inversely proportional. As the normal shock moves upstream, the throat shock moves downstream. This motion is illustrated in Fig. 3.13. Figure 3.13 shows two times: $t_{s h \text {,peak }}^{*}=-3.39$, before the normal shock reaches its maximum upstream position, and after at $t_{\text {sh,peak }}^{*}=0.31$. Mach contours are seen in Figs. 3.13a and 3.13b, which show a downstream motion of the throat shock and an increase in size of the throat expansion region in time. These effects are also illustrated in Figs. $3.13 \mathrm{c}$ and $3.13 \mathrm{~d}$ with pressure contours. This low frequency response to the normal shock motion appears to be slightly delayed in that the farthest downstream position of the throat shock 
is reached later in time than the farthest upstream position of the normal shock. This delay is consistent with the unsteady experimental pressure traces in Fig. 3.12b where the local pressure minimum in the throat tap occurs after the local maximum in the upstream tap.

\subsection{Shock Oscillation and Wave Dynamics}

By investigating the pressure traces at various streamwise locations along the centerbody surface, the fundamental dynamics of the acoustic compression waves are developed. Figure 3.14 shows a simplified version of several key moments of the wave propagation. At a MFR of 0.96, the inlet exhibits a generally steady normal shock seen in Fig. 3.14a. There exists a second, smaller shock at the geometric throat due to the sudden expansion and acceleration from flow turning after passing through the initial normal shock. A diffuse front high pressure wave in Fig. $3.14 \mathrm{~b}$ begins downstream of the AIP, and propagates upstream at an acoustic velocity through the subsonic diffuser. It continues upstream, moving over the transonic region near the throat shock, until it reaches the normal shock and causes an upstream motion in Fig. 3.14c. The spillage rate increases in Fig. 3.14d as the shock is farthest away from the cowl lip during its oscillation, expelling the high pressure gas from within the cowl. The upstream motion of the normal shock increases its strength, creating a lower Mach number leading into the expansion fan and thus increasing the throat shock strength as well. The upstream motion of the normal shock also causes a slightly delayed downstream motion of the throat shock. Once enough gas from the wave has been expelled, the pressure gradient across the normal shock decreases and the shock begins to recede in Fig. 3.14e. The strength of the throat shock and the size of the throat expansion also decrease as the normal shock moves downstream until the pressure gradient balances and a post-event state is reached in Fig. 3.14f with a stabilized normal shock. 


\subsection{Conclusions}

Pressure fluctuations of a low-boom inlet have been investigated experimentally and computationally. Unsteady experimental surface pressure traces at various streamwise locations along the inlet centerbody have revealed high pressure waves beginning downstream and propagating upstream through the subsonic diffuser. A DES methodology was able to accurately predict one such wave, however, only a short time integration period is calculated due to computational resource limitations and the repeatability of these waves is uncertain. Timeaveraged DES acoustic wave displacement curves show reasonable agreement with displacement curves of experimentally observed pressure peaks, indicating the compression waves are likely of an acoustic type. The mechanics of the pressure wave extracted from the DES illustrate the upstream motion of the wave resulting in an oscillation of the normal shock. Evidence of a secondary lambda shock wave located at the geometric throat is shown by both DES contour plots and by high amplitude fluctuations of the experimental data at the near-throat Kulite tap. Due to the presence of the throat shock wave, a transonic region exists just above the centerbody at the geometric throat location. This transonic region prevents the upstream-running acoustic wave from directly affecting the geometric throat region. This secondary shock is observed to respond to oscillations of the upstream external shock, but was not observed to have dynamics in response to the upstream-running acoustic waves.

A substantial increase in DES time integration is recommended for future studies. Only one significant shock oscillation is observed by the 0.1 seconds of DES data, while approximately 30 are seen in the five seconds of experimental data. Additional analysis is needed to fully understand the shock dynamics at the throat. 


\subsection{Figures and Tables}

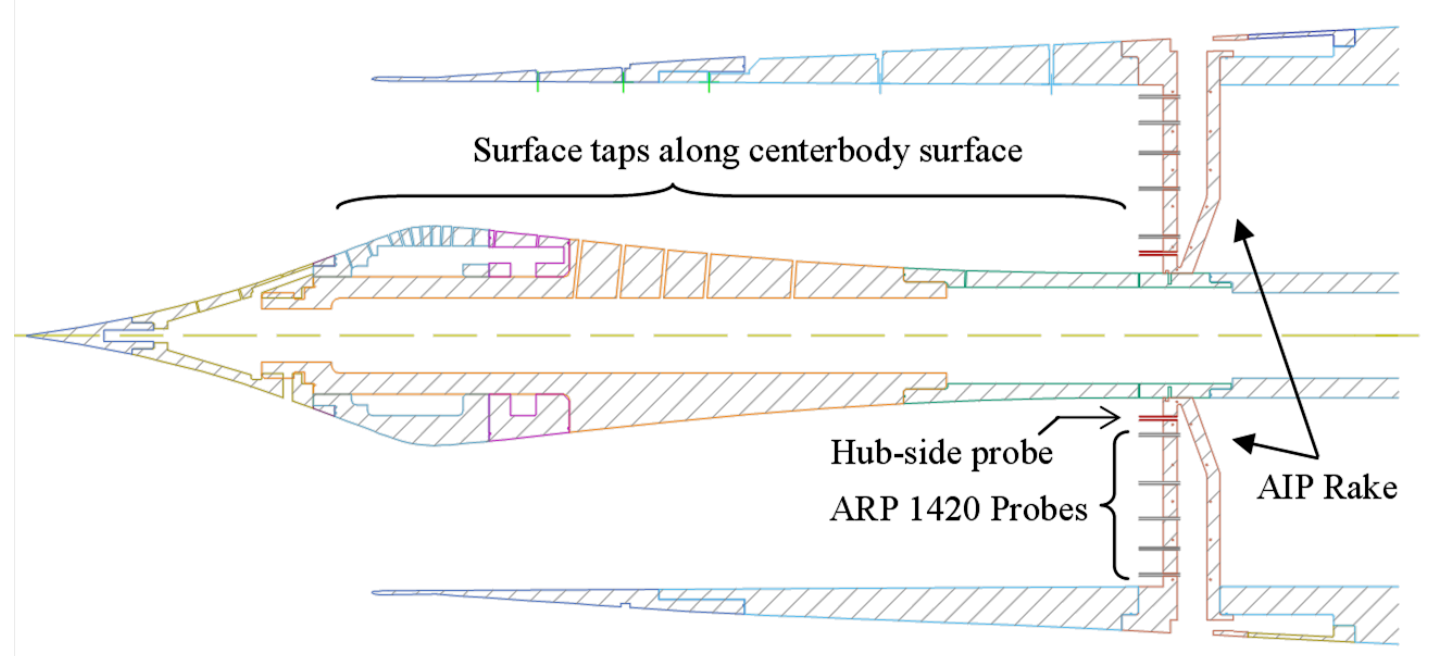

Fig. 3.1: Cross sectional view of experimental configuration used in the $8^{\prime} \mathrm{x}$ 6' supersonic wind tunnel at NASA Glenn Research Center. Centerbody surface and rake pressure tap locations are highlighted. 


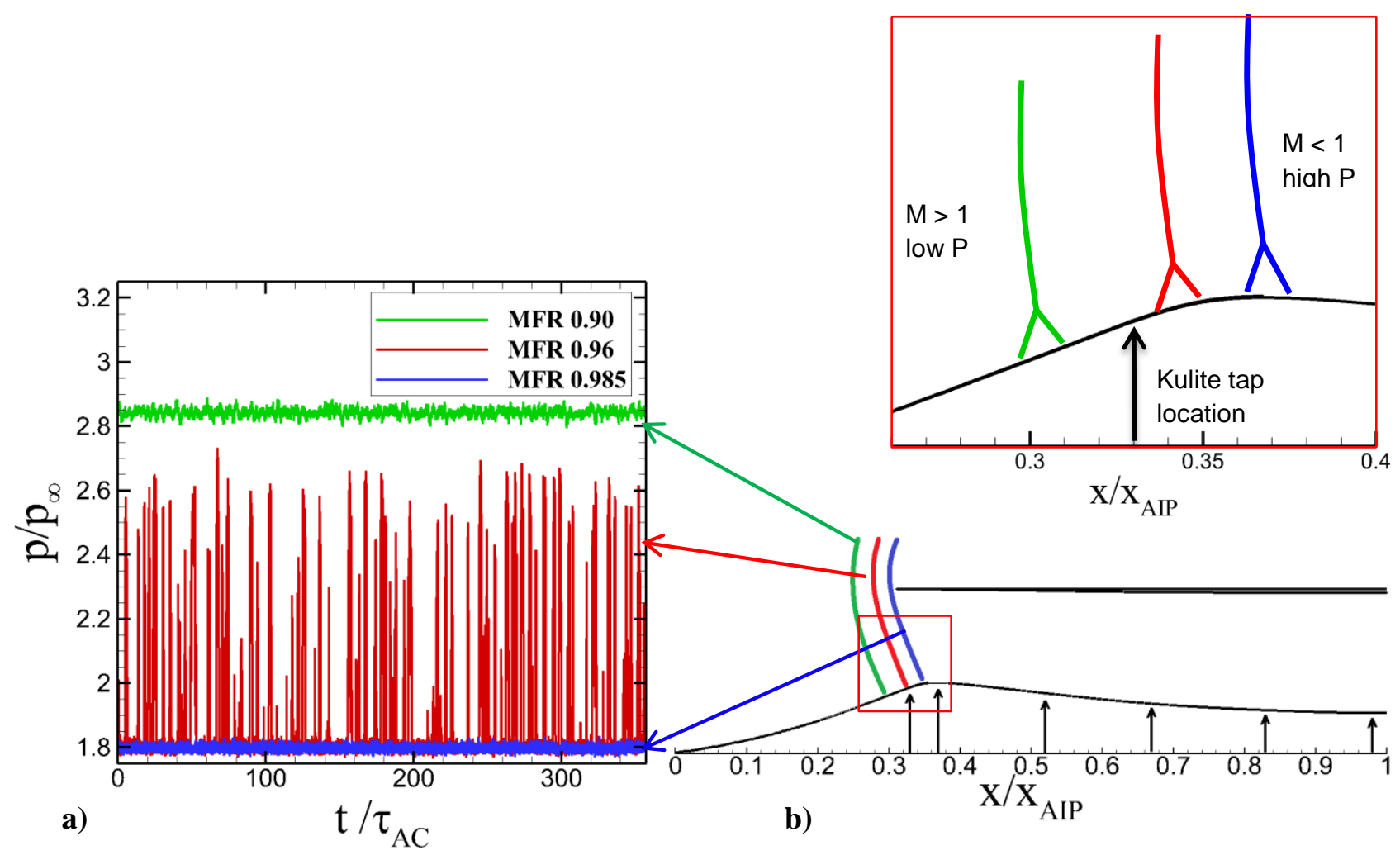

Fig. 3.2 Three different MFRs are shown with their: a) respective pressure trace at the upstreammost Kulite tap located at $x / x_{A I P}=0.33$ and $\left.\mathbf{b}\right)$ the approximate normal shock locations in relation to the streamwise Kulite taps which are indicated by black arrows at $x / x_{A I P}=0.33,0.37$, $0.52,0.67,0.83$ and 0.98 . 



Fig. 3.3: Contour plots of DES pressure fluctuations RMS values at the TDC plane illustrating shock position at a MFR of 0.94 a) extending from centerbody spike tip to the AIP and b) at the near-throat region. The high resolution DES tap is located at $x / x_{A I P}=0.29$ and is indicated with a red arrow, while the experimental taps are located at $x / x_{A I P}=0.33$ and 0.37 . 

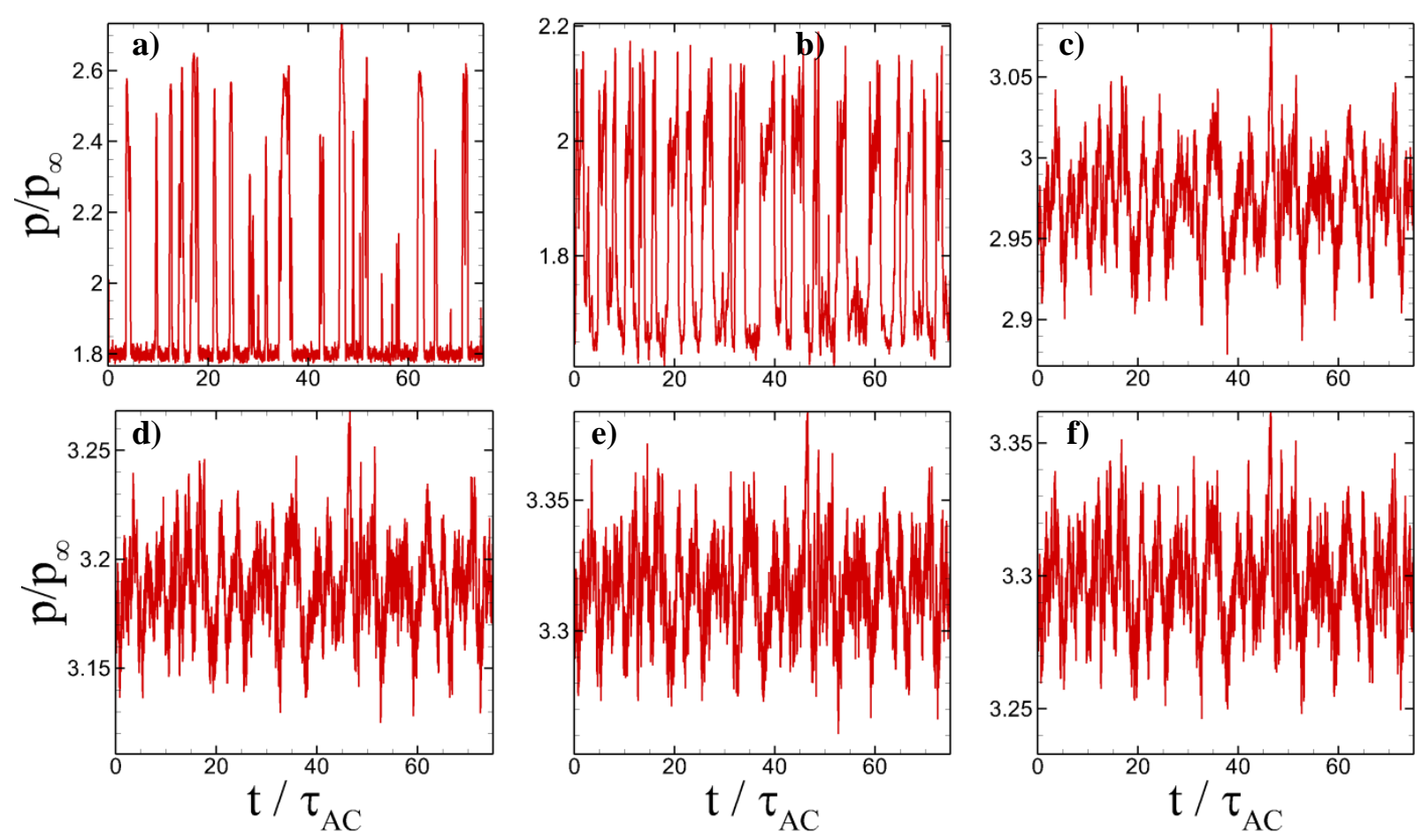

Fig. 3.4: Long-time experimental pressure traces of the MFR 0.960 case at: a) $x / x_{A I P}=0.33$ illustrating normal shock oscillations, b) $x / x_{A I P}=0.37$ illustrating high amplitude oscillations near the geometric throat, and $x / x_{A I P}=$ c) 0.52 , d) 0.67 , e) 0.83 , and f) 0.98 , illustrating sawtooth like oscillations at the downstream diffuser taps. 

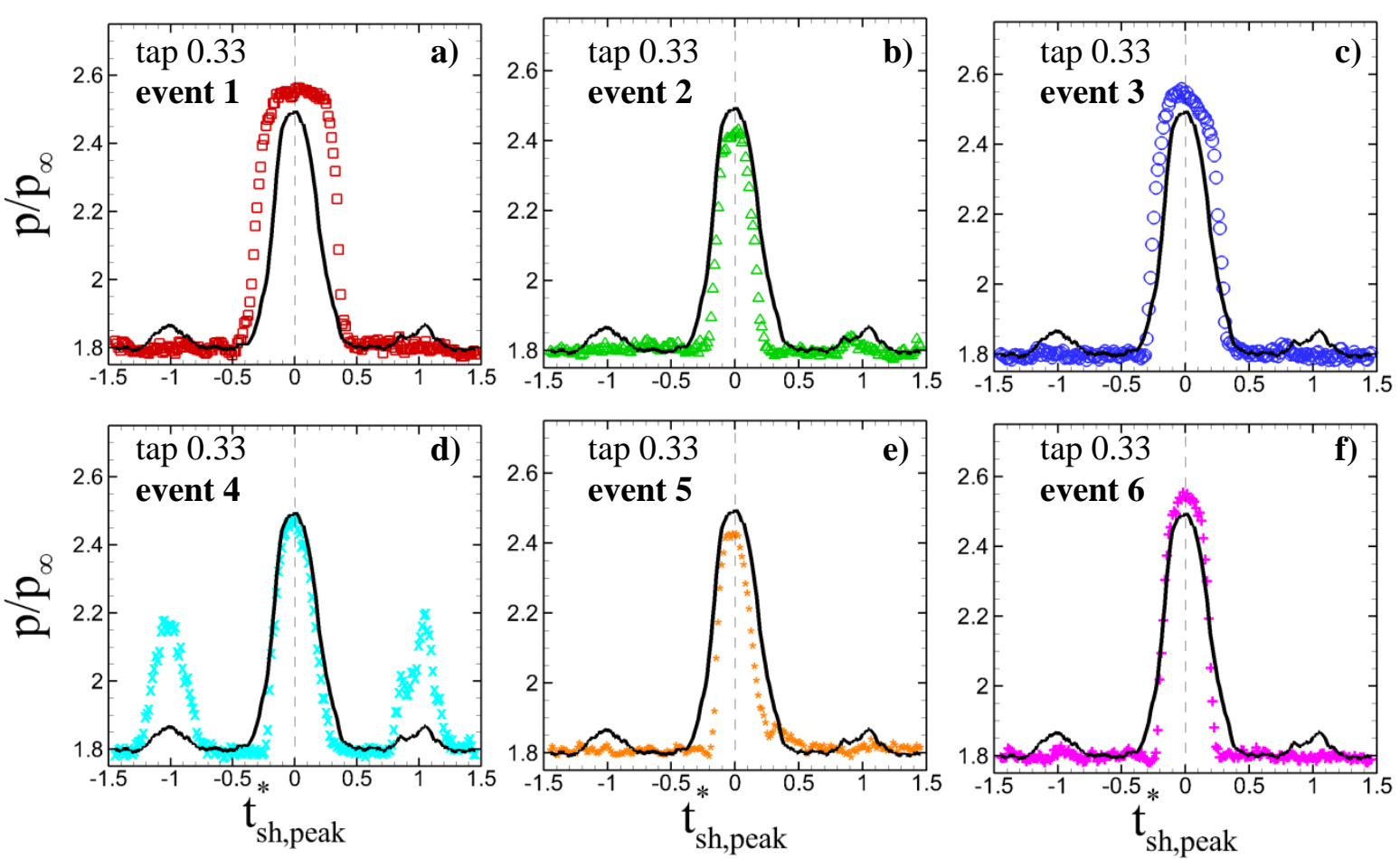

Fig. 3.5: Normalized centerbody surface pressure events occurring at $x / x_{A I P}=0.33$, just upstream of the normal shock at six different events in time. The time axis is normalized for the origin to correspond to the peak upstream normal shock position. 

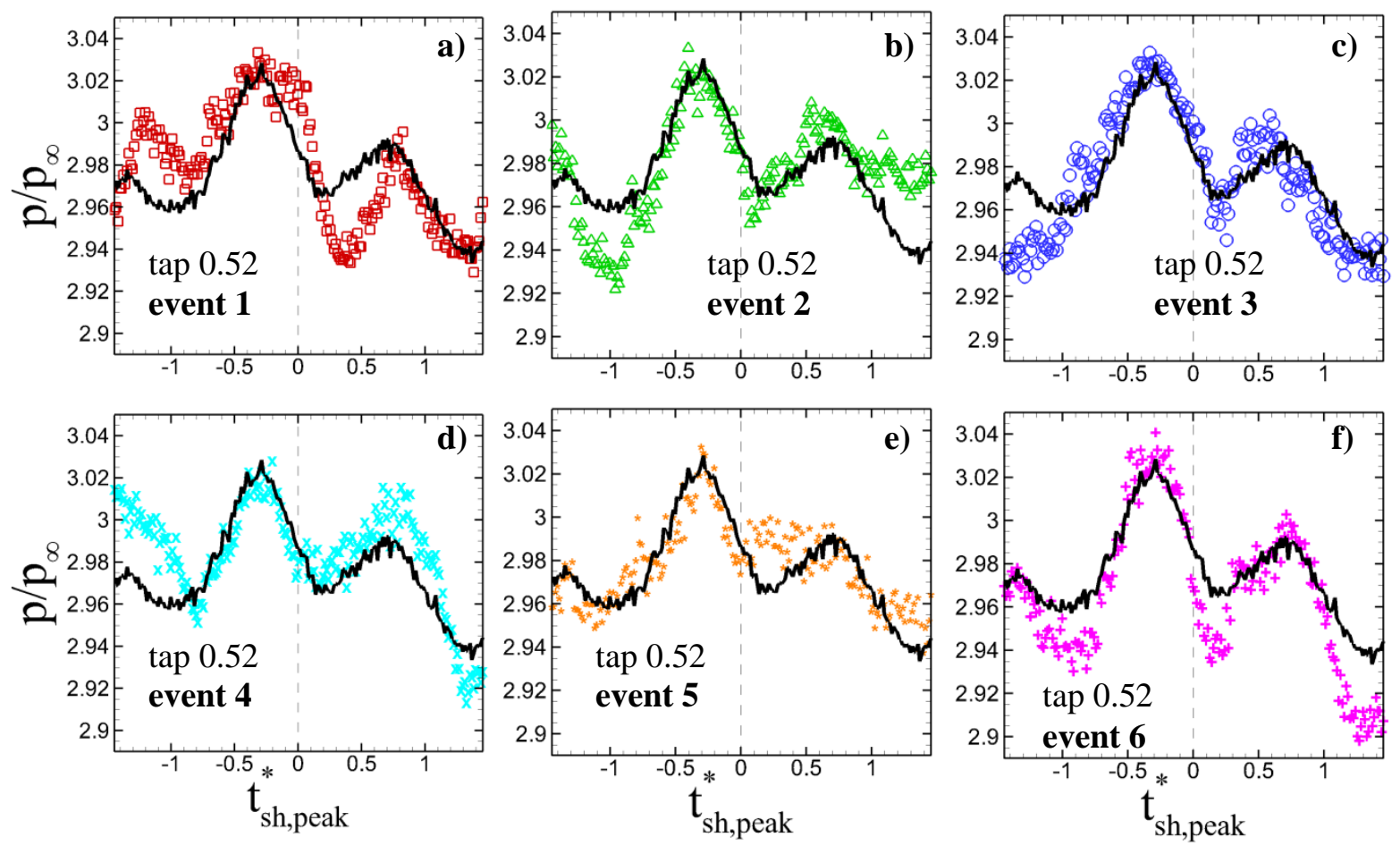

Fig. 3.6: Normalized centerbody surface pressure events occurring at $x / x_{A I P}=0.52$, where a saw-tooth like trace is observed. The normalized time origin of $t_{s h \text {,peak }}^{*}=0$ corresponding to the peak upstream normal shock position intersects the pressure at approximately $70 \%$ down the local downslope. 



Fig. 3.7: Normalized centerbody surface pressure events occurring at $x / x_{A I P}=0.98$, just upstream of the AIP. The normalized time origin of $t_{s h \text {,peak }}^{*}=0$ corresponding to the peak upstream normal shock position intersects at a local minimum. 

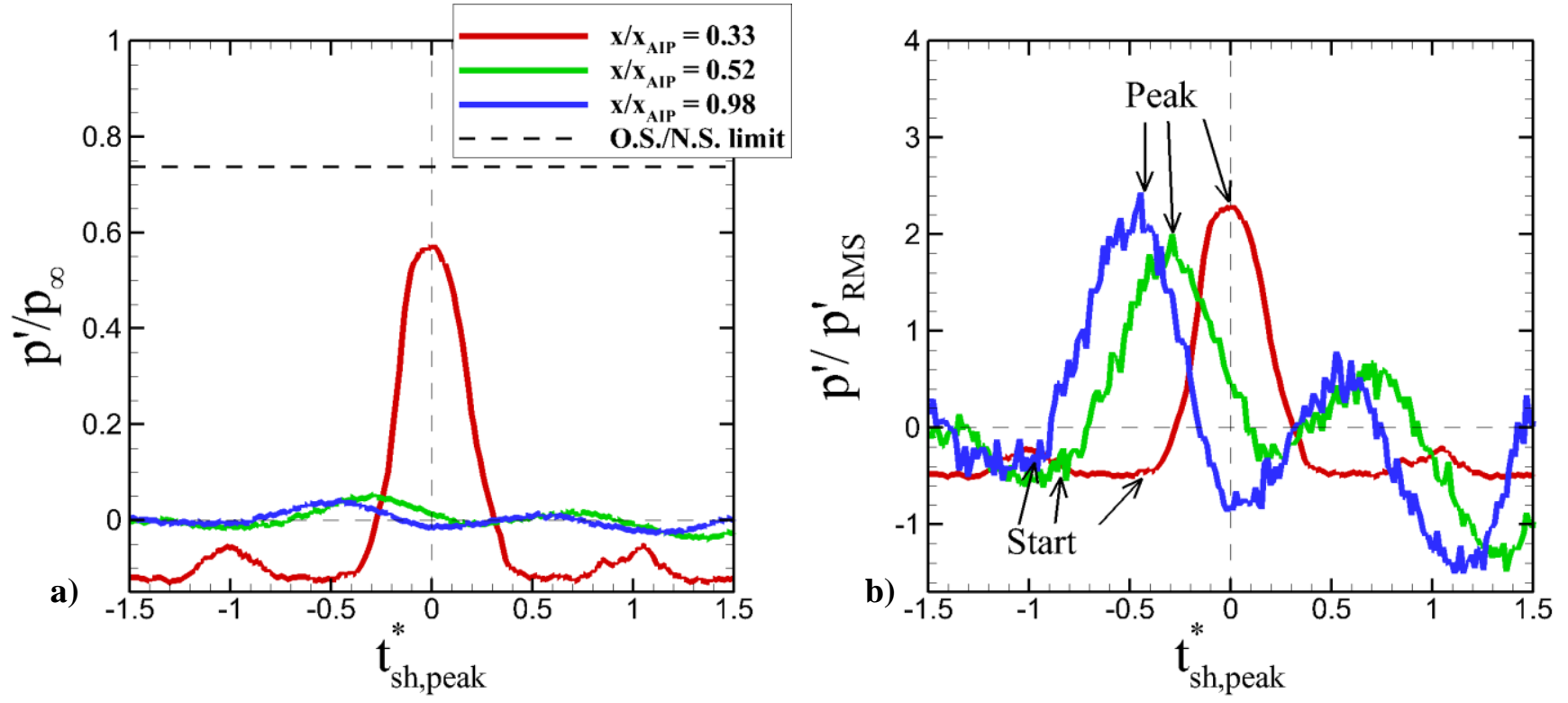

Fig. 3.8: Normalized experimental pressure fluctuations at $x / x_{A I P}=0.33$ (red), 0.52 (green), and 0.98 (blue). a) The increased amplitude of the pressure oscillations at the upstream-most tap due to the normal shock is clear, with the theoretical pressure value downstream of a 1D oblique shock normal shock structure plotted for reference. b) The time phase shift between streamwise locations is clearly illustrated after normalizing fluctuating pressure traces by their respective $p_{R M S}^{\prime}$ values, with both the "start," and "peak," times first occurring at $x / x_{A I P}=0.98$, then at $x / x_{A I P}=0.52$ and finally at $x / x_{A I P}=0.33$. 

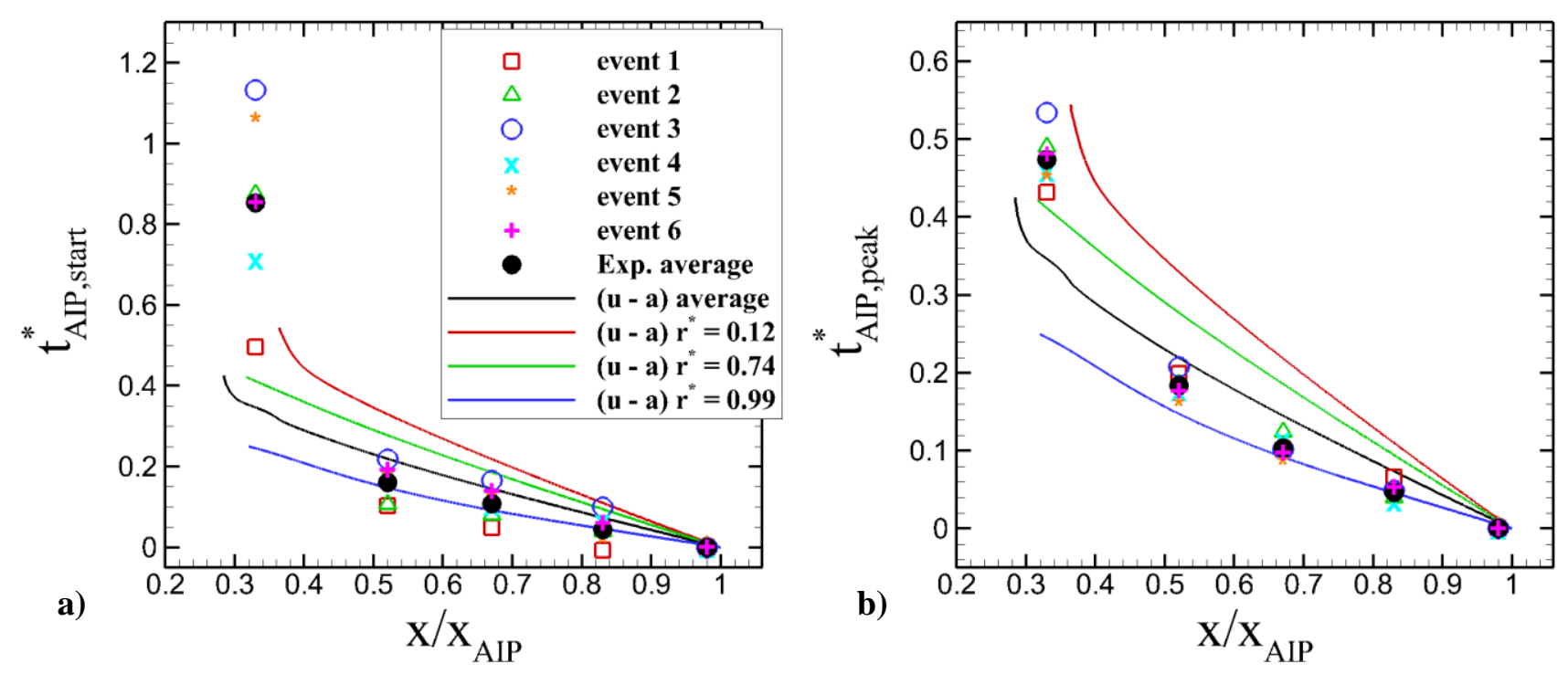

Fig. 3.9: Compression wave normalized time versus streamwise position distributions for six experimental pressure waves and their average. Identical distributions are generated using DES acoustic velocities at various wall normal heights as well as a spatial average from the centerbody to the inner cowl surface. The time scale is set for an origin at the a) start times of the near-AIP tap and the b) peak pressure times of the near-AIP tap. 


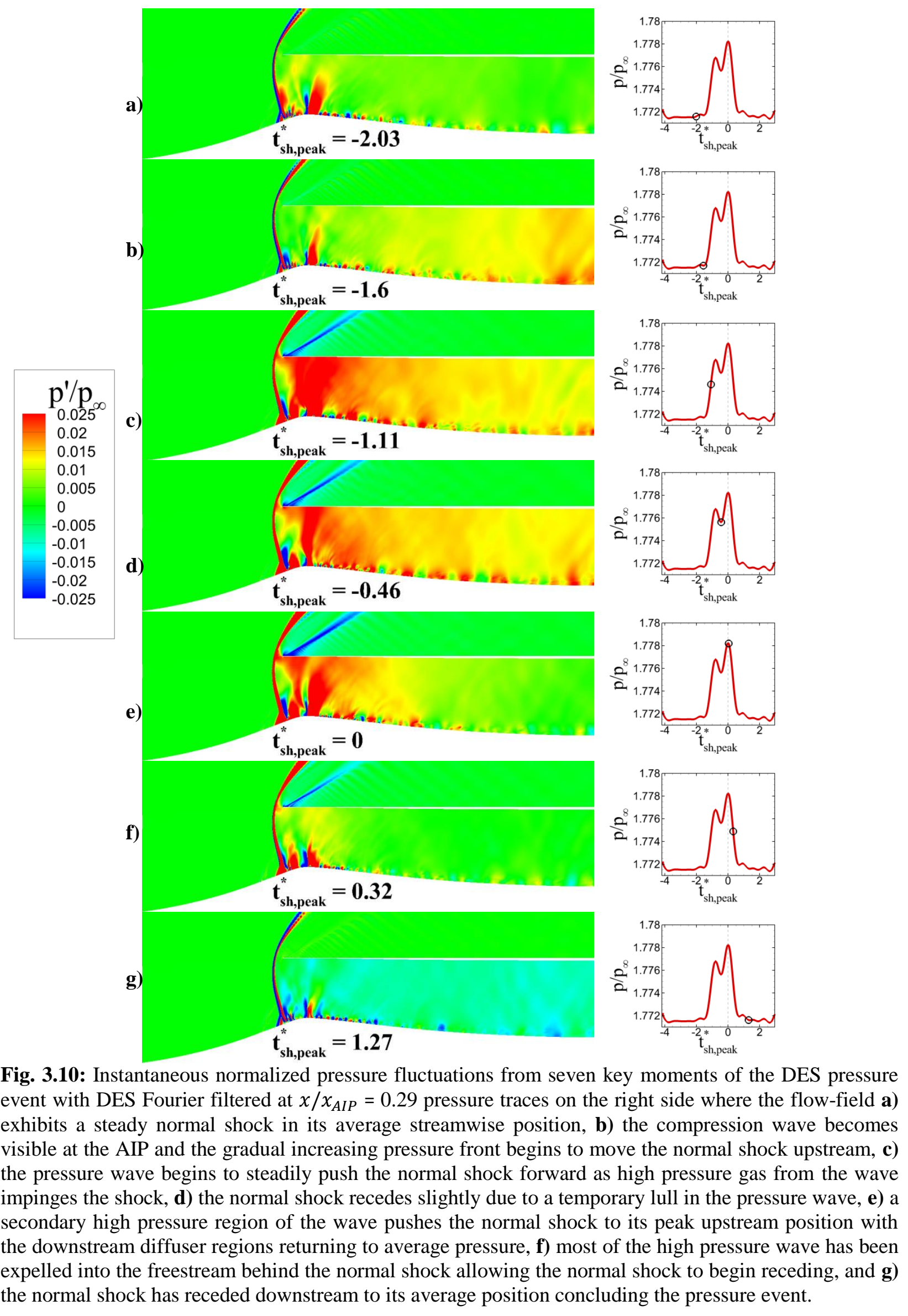


Fig. 3.11 (video): Contour plots of normalized pressure fluctuations predicted by DES at the TDC plane with frames are output every $2.8 \mathrm{e}-7 \tau_{A C}$ (or $2 \mathrm{e}-5$ seconds). The Fourier filtered DES upstream trace is seen in the upper right hand corner to illustrate current normal shock position. 

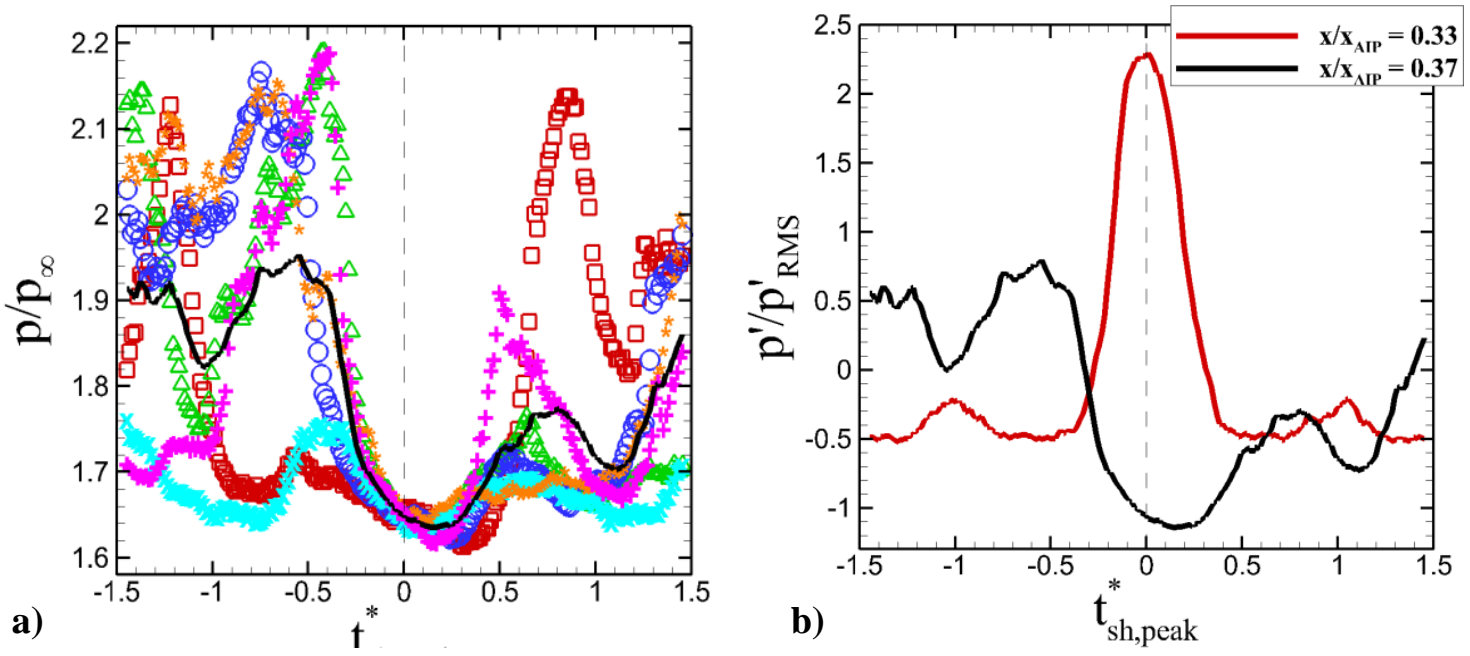

Fig. 3.12: Examining the near-throat pressure with: a) the experimental trace located at $x / x_{A I P}=$ 0.37 with the six separate pressure events and their average in black. b) Averaged pressure event curves from the $x / x_{A I P}=0.33$ (red) and $x / x_{A I P}=0.37$ (black) locations normalized by their respective $p_{R M S}^{\prime}$ values are compared illustrating a pressure minimum at $x / x_{A I P}=0.37$ occurring after the upstream pressure has peaked. 

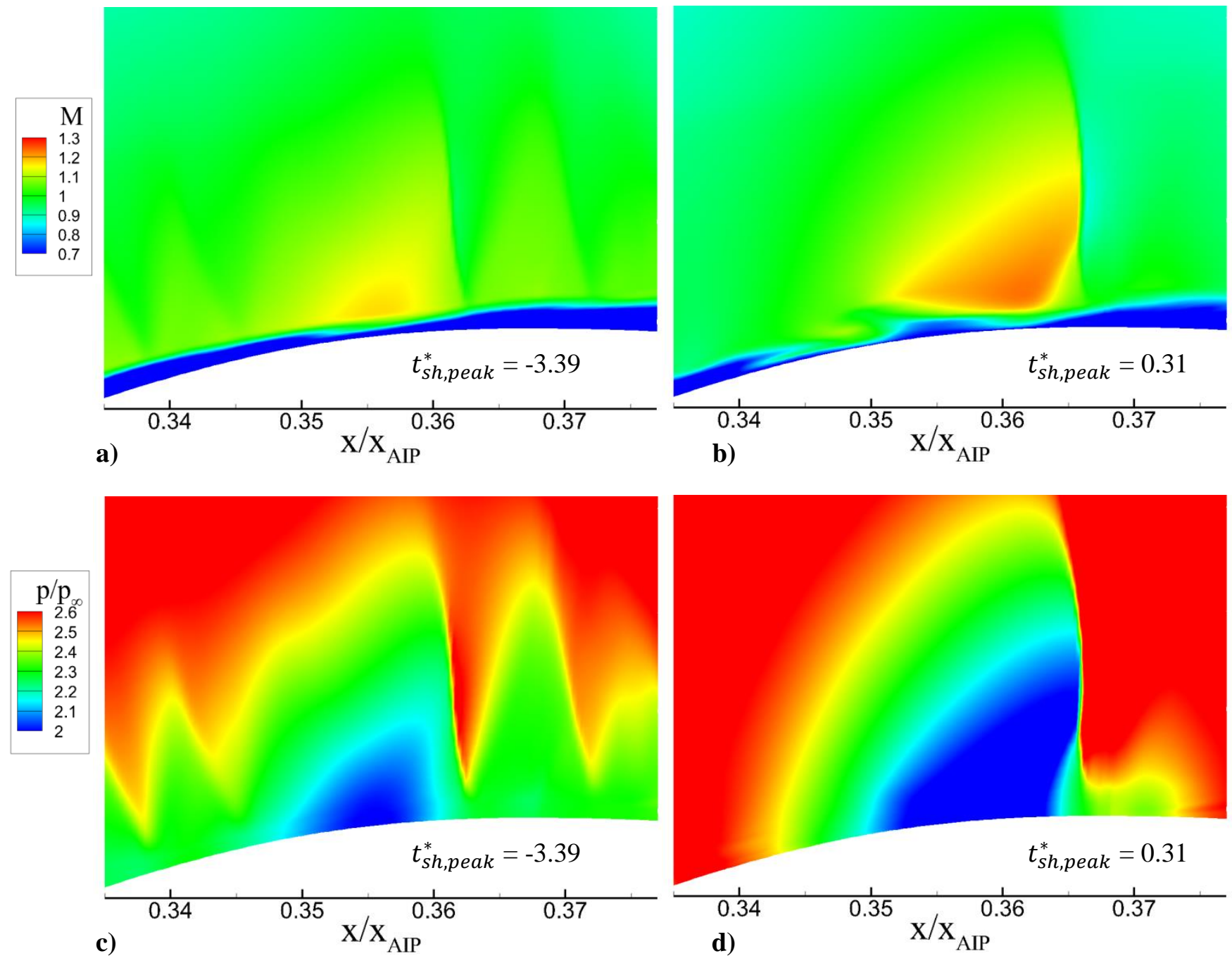

Fig. 3.13: Throat expansion and shock illustrated with: a) Mach contours before the pressure wave at $t_{s h, p e a k}^{*}=-3.39$ and $\mathbf{b}$ ) after the wave at $t_{s h, p e a k}^{*}=0.31$. This region is also illustrated by: c), d) pressure contours at the identical $t_{s h, p e a k}^{*}$ values. 

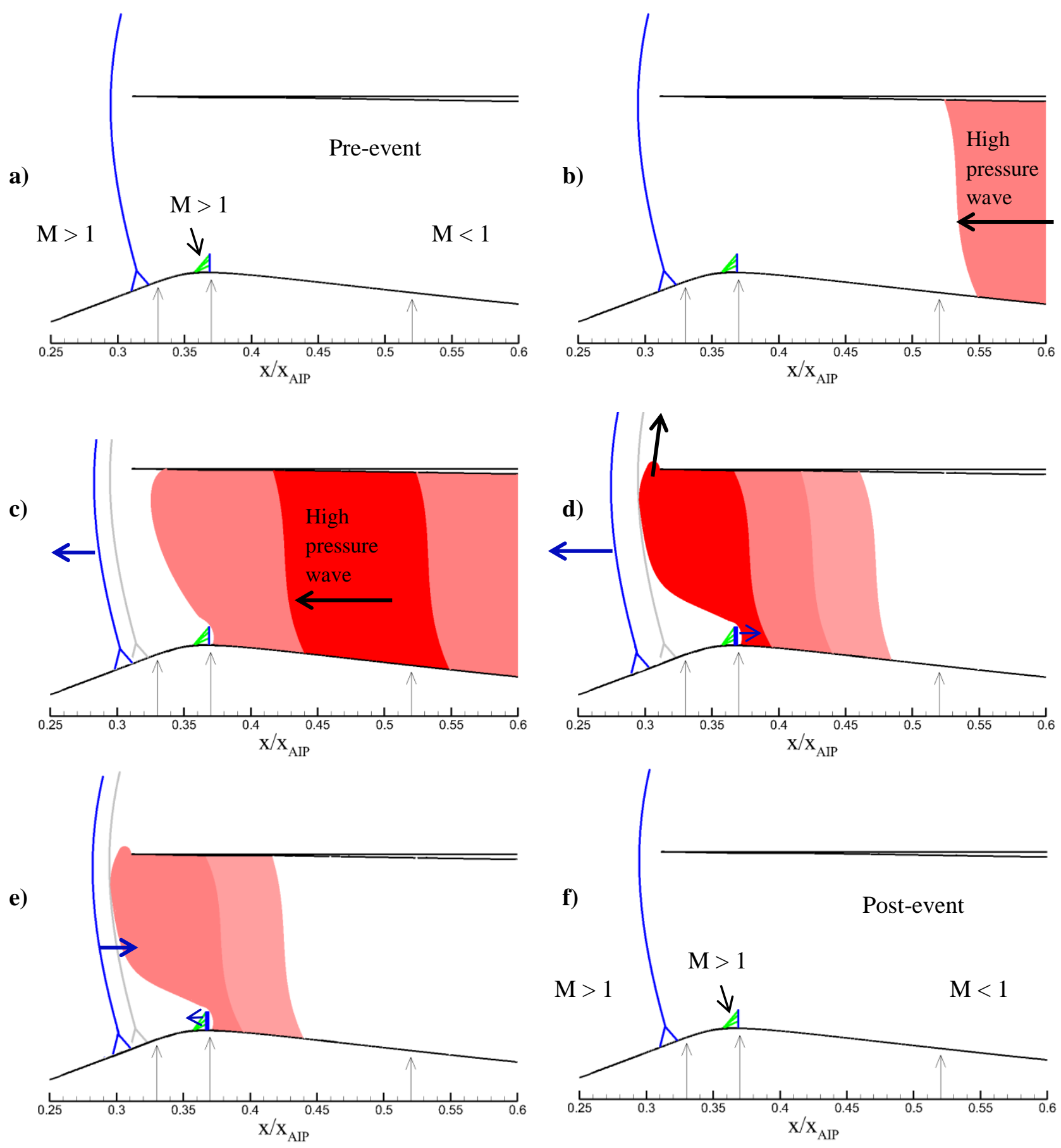

Fig. 3.14: Diagram of acoustic wave propagation and resultant normal shock motion. The inlet is a) initially in a steady state with a stable normal shock, b) the pressure wave begins propagating upstream, c) the pressure wave makes contact with and pushes the normal shock upstream generally avoiding the transonic region around the centerbody throat. The forward motion of the normal shock causes an increase in strength of the throat shock. d) The throat shock moves slightly downstream and the high pressure wave is expelled past the cowl lip into the freestream, allowing e) the normal shock to recede back to its average location, reducing the throat shock strength and f) a steady state is resumed. 
Table 3.1: Pressure event description and characteristics

\begin{tabular}{|c|c|c|c|}
\hline Event & $\boldsymbol{t}_{\text {sh,peak }} / \boldsymbol{\tau}_{\boldsymbol{A C}}$ & $\boldsymbol{p}_{\text {peak }} / \boldsymbol{p}_{\infty}$ & $\left(\boldsymbol{t}_{\text {sh,end }}-\boldsymbol{t}_{\text {sh,start }}\right) / \boldsymbol{\tau}_{\boldsymbol{A C}}$ \\
\hline 1 & 18.0 & 2.56 & 0.957 \\
\hline 2 & 70.7 & 2.42 & 0.517 \\
\hline 3 & 222.2 & 2.55 & 0.705 \\
\hline 4 & 249.6 & 2.48 & 0.574 \\
\hline 5 & 282.8 & 2.41 & 0.465 \\
\hline 6 & 307.3 & 2.54 & 0.521 \\
\hline average & - & 2.49 & 0.623 \\
\hline
\end{tabular}




\section{Chapter 4: Spectral Analysis and Mass Flow Rate Effects}

\subsection{Introduction}

Supersonic inlet performance is heavily dependent on the location and behavior of strong normal and oblique shock waves. A significant coupling exists between streamwise shock position and downstream diffuser mass flow rate. At sufficiently low mass flow rates, high amplitude oscillations of streamwise normal shock position begin in an event known as inlet buzz. Inlet buzz is detrimental to inlet performance by drastically reducing pressure recovery and in some cases may cause structural damage. It is crucial for any supersonic inlet to be designed with these features in mind.

Inlet buzz produces high amplitude shock oscillations in the presence of low mass flow rates in most inlets but otherwise can be quite unique to each inlet. Boundary layer separation, diffuser length, and diffuser sound speeds are all factors in the onset and frequency of shock oscillations. Fisher et al. ${ }^{17}$ were the first to categorize two different types of inlet buzz. Typically on-design mass flow rates will be associated with stable normal shocks and the greatest pressure recovery, but slight decreases in mass flow rate can cause the onset of "little" buzz. Little buzz produces small to modest amplitude oscillations, and has been shown to be caused by an ingestion of a shear layer within the cowl lip ${ }^{17,18,20}$. The shear layer is generated by the intersection of the oblique shock stemming from the centerbody spike tip and the normal shock, whose radial location decreases as the normal shock moves upstream. Some inlets have shown a range of mass flow rates below on-design conditions where little buzz is seen, followed by a period of relative shock stability, before a lower limit is reached and "big" buzz ${ }^{19}$ begins. Flow separation on the compression spike due to the shock wave boundary layer interaction (SWBLI)

has been shown to be a major cause of big buzz ${ }^{2,3,17,19,20}$. Most analyses of supersonic inlets will 
address inlet buzz but commonly will not distinguish between the little and big types. Typically it is big buzz that researchers refer to since it is the more violent of the two types which creates cause for more concern.

The current study aims to investigate the effect of mass flow rate on normal shock and surface pressure unsteadiness. Data collected at the $8^{\prime} \times 6^{\prime}$ supersonic wind tunnel at NASA Glenn Research is analyzed by Rybalko ${ }^{2}$ who uses schlieren video to determine normal shock streamwise location as a function of time. This data is presently considered and analyzed further. Unsteady Kulite pressure taps will provide five seconds worth of pressure readings at six different streamwise locations. Various mass flow rates will be considered with emphasis on near-buzz, near-design, and on-design conditions. The unsteady data will be converted from the time domain to the frequency domain using power spectral densities (PSD) to reveal the dominant wave frequencies, which may shed light on the physical mechanisms causing unsteadiness in the inlet flow-field.

\subsection{Experimental Methodology}

Experimental wind tunnel tests were performed at NASA Glenn Research Center in the fall of 2010. A significant portion of the wind tunnel investigation was dedicated to evaluating subsonic and supersonic vortex generators intended for control of the diffuser boundary layer. The current study does not share this focus and only baseline configurations (without vortex generators) will be considered. The 12 " scale inlet model is mounted upstream of a cold pipe separated by the aerodynamic interface plane (AIP). The cold pipe leads downstream to the mass flow plug whose streamwise location is altered to control mass flow rate. A diagram of the experimental setup is seen in Fig. 4.1 extending from the inlet model downstream to the mass flow plug. A convenient parameter is established to normalize mass flow rate by that of zero 
spillage conditions and defined as the mass flow ratio (MFR). Data for a wide range of MFRs is collected experimentally to evaluate inlet performance and study the MFR effects on varies flow aspects. A series of MFRs are considered in the current study, ranging from near-buzz conditions at 0.505 MFR to on-design 0.985 (corresponding to $1.5 \%$ spillage) conditions. Schlieren video is recorded at 2,000 frames per second for capturing shock location and dynamics while a series of surface taps and rakes collect static pressure measurements. The analysis in the current Chapter focuses on the schlieren video and unsteady surface pressure trace data. Kulite pressure transducers are placed at six streamwise locations along the inlet centerbody referenced by red arrows in Fig. 4.2a, and collect unsteady pressure readings at a $5 \mathrm{kHz}$ sampling rate using a Dewetron data system. A more detailed description of the experimental set up can be found in Hirt et al. ${ }^{6}$. These unsteady pressure measurements are used to evaluate pressure fluctuations up and downstream (depending on MFR) of the normal shock and throughout the subsonic diffuser. Fig. 4.2a shows the relative location of the normal shock for near-buzz (red), near-design (green), and on-design (blue) conditions with the lambda structures illustrated. Figure 4.2b shows the pressure traces from the upstream-most Kulite tap located at $x / x_{A I P}=0.33$ for the three MFRs considered. At the near-buzz condition the normal shock is significantly farther upstream than at the two higher MFRs, which results generally high subsonic pressures with high amplitude oscillations. Characteristic of the lambda shocks, separation of the boundary layer occurs just downstream which is a likely cause of these pressure oscillations. The size of this separated region has been shown to strongly influence the onset of big buzz ${ }^{17,20}$. At near-design conditions, the lambda structure is on average just downstream of the $x / x_{A I P}=0.33$ tap, enabling the capture of shock oscillations that are illustrated by local peaks in pressure. While the lambda shock is downstream of the tap, the flow is supersonic and the measured pressure is 
low. When the shock oscillates upstream and the lambda shock passes over the tap the pressure substantially increases and produces pressure peaks (see Fig. 4.2b green curve), illustrating the shock motion. Pressure traces at this Kulite tap are seen in Fig. 4.3a for several cases with MFR $\geq 0.90$. The cyan curve shows a MFR of 0.90 that exhibits a normal shock that is completely upstream of the $x / x_{A I P}=0.33$ tap. As the MFR increases, the normal shock moves to just downstream of the tap where shock oscillations are captured and illustrated by high amplitude pressure fluctuations in Fig. 4.3a for MFRs 0.952 and 0.96. At an even higher, on-design MFR of 0.985 , the normal shock is completely downstream of the Kulite tap and the resultant trace is low (supersonic) and steady. Each Kulite data set consists of five seconds of data which are converted into PSD plots to reveal dominant wave frequencies. Measureable frequencies will be up to $2.5 \mathrm{kHz}$ corresponding to the Nyquist frequency, or half the sampling rate. Using the PSD plots, the dominant frequencies will provide valuable information towards determining the mechanisms causing the pressure fluctuations.

\subsection{Schlieren Shock Tracking}

Chapter 3 showed the use of unsteady surface pressure readings to track normal shock oscillations at a 0.96 MFR which proved effective, however, this method was useful only at that specific MFR due to the static location of the Kulite taps and the dynamic motion of the normal shock with respect to MFR. Thus, a different method of shock tracking is required for varying MFRs. The outward-most radial portion of the normal shock extends upstream of the cowl lip and out into the freestream (by definition of external compression) and is captured by the schlieren video. Frames from schlieren at MFR $=0.955$ are seen in Fig. 4.4 that illustrate normal shock motion where a dashed vertical line is overlain on each frame to mark the approximate average location of the shock. Rybalko ${ }^{2}$ uses the schlieren video data to extract the streamwise 
normal shock location from each video frame. Figure 4.5 illustrates this process, which is automated using MATLAB $^{\mathrm{TM}}$ to convert each video frame into an intensity matrix. A line distribution at the radial distance corresponding to most upstream portion of the shock (corresponding to a vertical tangent line) in each frame is extracted as seen in Fig. 4.5c, revealing low intensity regions at the shock and at the cowl. Shock location can be determined by the distance between the shock and the known streamwise location of the cowl. The Nyquist frequency of the schlieren is $1 \mathrm{kHz}$ stemming from the 2,000 frames per second recording. This is lower than the unsteady pressure trace readings and means the schlieren video will only provide information on frequencies below $1 \mathrm{kHz}$.

Three schlieren video sets are analyzed: $\mathrm{MFR}=0.505,0.955$, and 0.982 . These were chosen as the "near-buzz", "near-design", and "on-design" cases, respectively. The actual ondesign MFR is 0.985 , but limited schlieren exists at this condition due to the shock steadiness observed at this MFR during preliminary testing. It is also difficult to observe the shock at such high MFRs due to its ingestion within the cowl where the schlieren video cannot investigate. Therefore, a slightly lower MFR of 0.982 was used but will still be referred to as "on-design," for simplicity. Near equivalent MFR surface pressure data sets are analyzed and compared to the schlieren, however slight MFR differences exists due to the unsteady nature of the inlet flow.

\subsection{Streamwise Shock Oscillations}

The streamwise normal shock location time trace data collected by Rybalko ${ }^{2}$ is herein compared with unsteady surface pressure traces. Rybalko ${ }^{2}$ extracts the average shock location from individually processed schlieren frames by tracking the upstream-most point of the normal shock in each frame and converting shock stand-off distance to a streamwise location based on 
known inlet dimensions. Shock location histograms from Rybalko ${ }^{2}$ are shown in Fig. 4.6 for the near-buzz (Fig. 4.6a), near-design (Fig. 4.6c), and on-design (Fig. 4.6e) MFRs. The effects of MFR are illustrated by comparing the left column of Fig. 4.6. At near-buzz conditions, Fig. 4.6a shows an average streamwise normal shock location of 5.2" downstream of the centerbody spike tip that oscillates between $x_{s h}=5.1$ and 5.27 equaling a 0.17 " range of motion. At near-design conditions, the position distribution spans an increased range between $x_{s h}=7.47$ " and 7.77" for a 0.3 " total range of motion. This is somewhat surprising since previous studies have shown the highest amplitude oscillations at lower MFRs during the big buzz regime $e^{2,3,17,18}$. However, Trapier et $a l .{ }^{20}$ show a short transitional period of decreasing MFRs that exhibit increasing oscillation amplitudes leading into biz buzz. A likely explanation of the increased range of motion at the near-design case is that the 0.505 MFR case is still in the transitional region where the oscillations have not yet grown to the high amplitudes of big buzz. This is also supported by the 0.505 MFR being greater than the 0.383 MFR lower limit observed by Hirt et al. ${ }^{6}$ at which continued reduction in MFR initiates (big) inlet buzz. The increased oscillation range at neardesign conditions may also indicate placement in the little buzz regime, which occurs at MFRs slightly below on-design conditions ${ }^{2,3,17,18}$. The normal shock is quite stable at on-design conditions in Fig. 4.6e which shows the shock located at approximately 7.8" downstream of the centerbody tip.

It is important to note that these trends are possibly due to the limited number of frames for each case. There are 1,000 schlieren frames for the near-buzz case, 7,000 frames for neardesign case, and only 485 for the on-design case. It is possible that increasing the number of frames may yield increased shock unsteadiness and it is in fact a matter of statistical significance. 
Perhaps if more frames were available at the near-buzz and on-design cases the shocks may be less stable.

Converting the normal shock position time trace into frequency space reveals the dominant frequencies of the shock motion. PSD plots are shown in the right column of Fig. 4.6 for each MFR considered. Figure 4.6b indicates a wide range of frequencies showing significant power at the near-buzz MFR. The two strongest frequencies occur in the lower range at 62 and $78 \mathrm{~Hz}$, while significant power is also seen at higher frequencies. Dussauge et $a l^{3}{ }^{3}$ report a dependence on separation length of normal shock oscillation frequency. They cite that the separation length based Strouhal $\left(S t_{\text {sep }}\right)$ number equal to 0.035 can be used with a known separation length $\left(L_{s e p}\right)$ and freestream velocity $\left(u_{\infty}\right)$ to determine the normal shock oscillation frequency due to unsteady post-shock flow separation effects. Due to the lack of computational results at similar MFRs, the separation frequency for the near-buzz condition cannot be calculated due to the unknown separation length. However, computational results exist for the remaining two MFRs. DES data previously presented in Chapters 2 and 3 predict the separation length downstream of the normal SWBLI, allowing the separation frequency at near-design 0.955 MFR to be calculated using the Strouhal number definition with

$$
\begin{gathered}
S t_{\text {sep }} \approx 0.035=\frac{f_{\text {sep }} L_{\text {sep }}}{u_{\infty}} \\
f_{\text {sep }}=\frac{(0.035)(493 \mathrm{~m} / \mathrm{s})}{0.0169 \mathrm{~m}}=1021 \mathrm{~Hz}
\end{gathered}
$$

Unfortunately, this frequency falls outside the measurable range of the current shock tracking method as it is greater than the Nyquist frequency (or half of the $2 \mathrm{kHz}$ sampling rate). However, the frequency of the acoustic mode (see section 2.10) can be predicted using the relationship between the diffuser acoustic propagation velocity and the acoustic length scale defined as the 
distance between the normal shock and the downstream mass flow plug. A three-dimensional spatial average of all zones completely downstream of the normal shock is used for the diffuser streamwise velocity and sound speed measurements. Dussauge et al. ${ }^{3}$ determine the acoustic Strouhal number to be approximately equal to 1 for general external compression inlets. The acoustic frequency for the near-design 0.955 MFR case based on these values is calculated as

$$
S t_{A C} \approx 1=f_{A C}\left(\frac{L_{A C}}{a+u}+\frac{L_{A C}}{a-u}\right)=f_{A C}\left(\frac{3.03 \mathrm{~m}}{474+136 \mathrm{~m} / \mathrm{s}}+\frac{3.03 \mathrm{~m}}{474-136 \mathrm{~m} / \mathrm{s}}\right) ; \Rightarrow f_{A C}=71.78 \mathrm{~Hz}
$$

A significant peak is seen at approximately $70 \mathrm{~Hz}$ in Fig. 4.6d illustrating an acoustic influence on the normal shock motion at near-design conditions. This is consistent with the results of Chapter 3 where acoustically induced shock oscillations are observed numerically. There exists a second peak in Fig. 4.6d at a lower $31 \mathrm{~Hz}$ that has a slightly greater power than the purely acoustic frequency. Altering the characteristic frequency derivation of equation (4.3) for a convective downstream, acoustic upstream wave yields a combined frequency of

$$
S t_{\text {comb }} \approx 1=f_{\text {comb }}\left(\frac{L_{A C}}{u}+\frac{L_{A C}}{a-u}\right)=f_{\text {comb }}\left(\frac{3.03 \mathrm{~m}}{136 \mathrm{~m} / \mathrm{s}}+\frac{3.03 \mathrm{~m}}{474-136 \mathrm{~m} / \mathrm{s}}\right) ; \Rightarrow f_{\text {comb }}=32.01 \mathrm{~Hz}
$$

This result indicates that the combined downstream, acoustic upstream convection mode likely causes the high PSD frequency of $31 \mathrm{~Hz}$ at near-design conditions. These two peaks indicate clear contributions of acoustic and combined convective/acoustic waves to normal shock unsteadiness at near-design conditions. However, these waves appear to be secondary drivers while the primary occurs at a $20 \mathrm{~Hz}$ frequency, which will be addressed in the following section. Moving to on-design conditions, the power of the shock oscillations across the frequency spectrum is significantly weaker than the 0.955 case, indicative of a more stable shock. Rybalko ${ }^{2}$ 
uses a short domain (extending only to the AIP) DES approach to solve for a baseline on-design flow case and similarly estimates separation, acoustic, and combined convective/acoustic frequencies to be 186,32 , and $26 \mathrm{~Hz}$, respectively. A larger numerically predicted separated region at on-design conditions significantly reduces the separation frequency by equation (4.1). Differences in the acoustic frequencies based on MFR arise from a higher streamwise diffuser velocity at on-design conditions leading to lower pressures (in agreement with Fig. 4.3) and therefore lower sound speeds. The increase in streamwise velocity and decrease in sound speed compound to drastically reduce the upstream running acoustic velocity term in (4.3), resulting in a much lower acoustic frequency when compared to near-design. Figure 4.6f shows the PSD of the on-design case illustrating high power density at $62 \mathrm{~Hz}$. This corresponds to approximately twice the estimated acoustic wave frequency suggesting it is possibly a $2^{\text {nd }}$ harmonic wave. Significant peaks are absent at the estimated separation frequency, indicating the shock instability at on-design conditions is dominated by other mechanisms. Rybalko et al. ${ }^{26}$ performed an experimental study of an inlet analogue external compression inlet SWBLI and found that the unsteady separation mechanism dominates the shock motion only when separation lengths exceed the throat clearance. The separation length for the current inlet is approximately half of the throat clearance at on-design conditions and even less for near-design, further suggesting a secondary role of separation in shock unsteadiness at near- and on-design conditions.

\subsection{Unsteady Surface Pressure Fluctuations}

Analysis of the near-design 0.955 MFR was limited due to the frame rate of the schlieren video which was too low to capture potential high frequency separation waves. However, higher frequency waves up to $2.5 \mathrm{kHz}$ are captured using unsteady pressure tap data taken at the centerbody surface. Data sets at near-buzz, near-design, and on-design conditions are evaluated 
with corresponding MFR of $0.505,0.952$, and 0.985 , respectively. The experimental Kulite taps are located at six different streamwise positions spanning from $x / x_{A I P}=0.33$ at the mostupstream and $x / x_{A I P}=0.98$ at the most-downstream. The most-upstream, the most-downstream, and the midstream tap at $x / x_{A I P}=0.52$ are selected for close examination of the inlet flow-field frequencies. Figure 4.7 shows near-buzz, near-design, and on-design MFRs in the top, middle, and lower rows, respectively, and streamwise locations at $x / x_{A I P}=0.33,0.52$, and 0.98 in the left, middle, and right columns, respectively. The pressure fluctuation PSD values are several orders of magnitude greater than those from the schlieren shock tracking method, indicating much stronger oscillations in surface pressure than normal shock oscillations. This is to be expected as the surface pressure is far more responsive to small scale turbulence than the normal shock is. Figures $4.7 \mathrm{a}, 4.7 \mathrm{~b}$, and $4.7 \mathrm{c}$ show a peak at $20 \mathrm{~Hz}$ for the near-buzz 0.505 MFR case at all three streamwise locations. Previous studies ${ }^{2,3,17,18}$ have shown that normal shock oscillations at low MFRs are characterized by flow separation scales. As previously mentioned, the estimation of separation frequency using the Strouhal number by Dussauge et al. ${ }^{3}$ requires a known separation length, which, for the near-buzz case, is unknown. Instead, the observed $20 \mathrm{~Hz}$ frequency can temporarily be assumed as a valid $f_{\text {sep }}$ in equation (4.1) to calculate the required separation length for this assumption to indeed be true. Equation (4.1) yields a required separation length of $L_{\text {sep }}=33.97$ " $(0.66 \mathrm{~m})$ for agreement with the temporarily assumed separation frequency. However, a separation due to the SWBLI of this length is impossible. The inlet throat occurs approximately $9.5 "(0.24 \mathrm{~m})$ downstream of the centerbody tip and acts as an upper $L_{s e p}$ limit. It is possible that the flow stays separated past the throat and produces a 33.97" separation bubble, but separation past the throat would be the result of increasing cross sectional area of the diffuser, not characteristic of the SWBLI induced separation on which $L_{s e p}$ of 
equation (4.1) is based. This emphasizes the classification of 0.505 MFR as "near-buzz," since it lacks a strong influence by the separation frequency that would likely occur at much higher frequencies than $20 \mathrm{~Hz}$ per equation (4.1). Strong peaks also occur at 39 and $60 \mathrm{~Hz}$ that are likely harmonics of the $20 \mathrm{~Hz}$ wave. Figure $4.6 \mathrm{~b}$ shows a peak at $60 \mathrm{~Hz}$ indicating that perhaps the third harmonic of the same mechanism causing pressure fluctuations is also causing the normal shock oscillations at near-buzz conditions.

Near-design conditions are illustrated at three streamwise positions in the second row of Fig. 4.7. At a 0.952 MFR, secondary contributors to pressure fluctuations are seen at the midstream and downstream taps in Figs. 4.7e and 4.7f. The peak at $65 \mathrm{~Hz}$ may be related to the estimated $72 \mathrm{~Hz}$ acoustic frequency from the previous section. The strongest wave affecting pressure fluctuations resonates at $18 \mathrm{~Hz}$ as illustrated in Fig. 4.7d-f. This $18 \mathrm{~Hz}$ value agrees closely with the $20 \mathrm{~Hz}$ seen at the near-buzz condition, suggesting that the mechanism causing these waves may be independent of MFR. A side-by-side comparison is made between the schlieren shock tracking data and the upstream-most pressure tap in Fig. 4.8 for the near-design MFR. Both PSD measures are normalized by their respective maximum PSD for a simplified intensity comparison. Logarithmic scales are used for both the $\mathrm{x}$ - and $\mathrm{y}$-axes which illustrate the greatest power density at $20 \mathrm{~Hz}$ in the schlieren videos in Fig. 4.8a. This dominant frequency appears in both measures of unsteadiness and shows significantly greater PSD than the remaining peaks in Figs. $4.8 \mathrm{a}$ and $4.8 \mathrm{~b}$. This suggests that a single mechanism dominates both the normal shock and surface pressure fluctuations at these conditions. High PSD indicated at 18 $\mathrm{Hz}$ in Figs. 4.7e and 4.7f also shows that this wave frequency dominates throughout the entire inlet flow-field. Trapier $e t$ al. $^{20}$ see a similar $20 \mathrm{~Hz}$ dominant frequency after investigation of an external compression inlet at a 1.8 freestream Mach number and near-design conditions. Their 
results illustrate the presence of this low frequency wave and its harmonics through a range of decreasing MFRs and into the big buzz regime, much like the current results. They conclude that due to constant frequency but varying shock oscillation amplitude with varying MFR, the $20 \mathrm{~Hz}$ frequency is likely related to acoustic propagation. A $20 \mathrm{~Hz}$ normal shock oscillation frequency is calculated in Chapter 2.10 by dividing the number of observed shock oscillations by the time window, and agrees with the $20 \mathrm{~Hz}$ observed in the power spectra. Chapters 2 and 3 illustrate direct correlations between acoustic wave propagation and normal shock oscillations, supporting a strong acoustic influence on flow unsteadiness. If this is in fact true, then the pressure fluctuation PSDs will add to the evidence supporting acoustically induced flow unsteadiness by the dominant $20 \mathrm{~Hz}$ frequency in both the near-design and near-buzz cases. Using the acoustic Strouhal number to estimate acoustic frequencies generally does not provide good agreement with what is observed by the pressure fluctuation and shock position PSD. This suggests that perhaps improper scales were used in the calculations of equations (4.1) - (4.4).

The comparatively lower PSD values at on-design conditions in the third row of Fig. 4.7 further illustrate the steadiness of the higher 0.985 MFR. A range of low frequency waves dominate the $x / x_{A I P}=0.33$ tap in Fig. $4.7 \mathrm{~g}$ that are unrelated to the $20 \mathrm{~Hz}$ frequency previously discussed. This is due to the downstream location of the normal shock in comparison to the upstream tap, meaning acoustic waves are unable to propagate this far upstream. Low frequency energy concentrations reappear at $x / x_{A I P}=0.52$ in Fig. $4.7 \mathrm{~h}$ but as secondary effects to energy dense waves at $750 \mathrm{~Hz}$. A reduced energy concentration is expected for the $20 \mathrm{~Hz}$ frequency as its relative strength decreases with increasing MFR. The cause of the high frequency wave at 750 $\mathrm{Hz}$ is unclear. It is unlikely to be caused by separation which is estimated to propagate at $186 \mathrm{~Hz}$ for on-design by the Strouhal number, and it is also unlikely to be caused by the estimated $32 \mathrm{~Hz}$ 
characteristic of acoustic propagation determined by equation (4.3). Another energy concentration is seen at $60 \mathrm{~Hz}$ at the $x / x_{A I P}=0.98$ location in Fig. 4.7i. It is possible that this is caused by an acoustic resonance that is characterized by a shorter $L_{A C}$. An example of such a length scale would include the streamwise distance from the normal shock to the increase in cross sectional area leading into the cold pipe. Due to the absence of an energy dense frequency corresponding to the separation length or characteristic acoustic frequencies, the dominant cause of unsteadiness at on-design conditions is inconclusive based on the unsteady pressure traces and schlieren video.

\subsection{Inlet Buzz Regimes}

Defining the lower MFR limit that marks the onset of inlet big buzz and the characteristic high amplitude normal shock oscillations is crucial for determining inlet operating conditions. It is also useful to know the MFRs corresponding to the little buzz regime so that negative effects associated with normal shock unsteadiness may be avoided when a lower MFR is required. The buzz regimes are best characterized by the amplitude of the normal shock oscillations which are easily captured using schlieren video. However, the majority of the collected schlieren video is at either low MFR buzz conditions, or at near-design 0.955 MFR conditions. These two MFR regimes produce the most unsteady normal shocks which were the primary focus of schlieren data acquisition, reducing emphasis and data for the intermediate range of MFRs. Root mean square (RMS) values of shock position normalized by the clearance height between the centerbody and cowl at the throat are presented as a function of MFR in Fig. 4.9a for the three MFRs. The on-design case proves to again be most stable, followed by the near-buzz case and finally the near-design exhibiting the most unsteady normal shock. Again, this is somewhat surprising since lower MFRs generally correspond to the greatest amplitude oscillations and 
overall increased unsteadiness. However, this may be explained by the limited number of nearbuzz schlieren frames compared to the near-design case. The assumption that 0.505 MFR is too high for placement in the big buzz regime where the greatest normal shock unsteadiness is seen still holds true. The high $x_{s h, R M S}$ value at MFR $=0.955$ along with evidence of acoustically dominated unsteadiness indicates that this MFR falls in the little buzz regime.

With the lack of schlieren data at intermediate MFR between near-buzz and near-design regimes, we again turn to the unsteady pressure readings to offer insight into the transition between little buzz and big buzz. Fluctuating pressure RMS values for eight different MFRs are plotted in Fig. 4.9b with different symbols representing various streamwise locations. In terms of pressure, the highest fluctuations occur at the lowest MFR of 0.505 where a slight increase in MFR corresponds to a significant reduction in pressure fluctuations. This indicates that a transitional region to the big buzz regime likely exists and begins at MFR $<0.6$. Transition extends below MFR $<0.505$ based on the conclusion that MFR $=0.505$ is not quite in the big buzz regime. The onset of big buzz is therefore at MFR $<0.505$, and a green shaded region illustrates this in Fig. 4.9b. The remaining MFRs in Fig. 4.9b show comparatively low pressure fluctuations with the exception of the squares and triangles at MFRs $=0.955$ and 0.96 . These two symbols correspond to the Kulite taps located at $x / x_{A I P}=0.33$ and 0.37 , which were shown in Chapter 2 to exhibit high pressure fluctuations due to oscillations of normal shocks across the taps. While the pressure fluctuations are higher at these streamwise locations, it is misleading because the unsteadiness is not higher in a frequency sense, but rather a magnitude sense due to unique shock positioning. Instead, only the four downstream taps are considered in the zoomed view of Fig. 4.9c where increased pressure unsteadiness is clear at the near-design MFRs. The fluctuations are substantially lower at the MFR between 0.6 and 0.8 , and the on-design 0.985 
case, suggesting that MFRs between approximately 0.85 and 0.97 encompass the little buzz regime.

\subsection{Conclusions}

Analysis of schlieren video and unsteady Kulite surface pressure tap data has revealed information about the mechanisms leading to flow-field unsteadiness in an external compression inlet at various mass flow rates. The on-design mass flow condition proved most stable in terms of both normal shock position and surface pressure fluctuations. The near-design case exhibited the most unsteadiness of the shock and pressure measures, indicating a strong presence of little buzz. The near-buzz conditions reveal an intermediate stability between the near- and on-design cases. Evidence is seen supporting acoustic waves as normal shock instability sources at neardesign conditions through power spectral density analysis. The pressure traces indicate a substantial dominance of a low $20 \mathrm{~Hz}$ frequency for both the near-design and near-buzz cases. This suggests the mechanism, which increases in intensity as the big buzz regime is approached, is independent of MFR. It is unclear whether this low frequency is driven by acoustic or separation modes, however, evidence exists that supports the former is more likely. Strong correlations between the separation length and normal shock and pressure unsteadiness were not observed in the three emphasized MFRs. Comparisons of fluctuating pressure values at various MFRs were used to estimate the approximate regimes corresponding to big and little buzz. Results indicate a highly unsteady region at MFRs between 0.85 and 0.97 , with stable regimes above and below until transition into big buzz occurs at MFR $<0.55$.

Further investigation of the low frequency $20 \mathrm{~Hz}$ mechanism is highly recommended as it appeared in most MFR cases as a significant source of pressure oscillations. Extending the current analysis to the low MFR, big buzz regime will reveal additional details about the low 
frequency wave and the source of the instability. Understanding this mechanism may provide information on its manipulation, potentially leading to flow control methods for increased flow stability in both little buzz and big buzz regimes. 


\subsection{Figures}

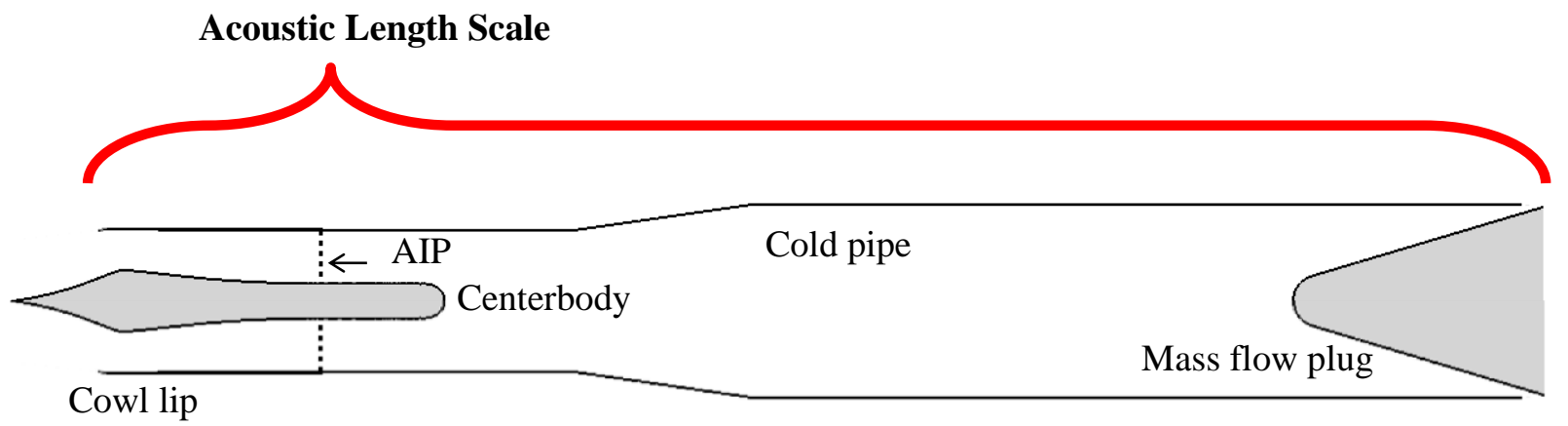

Fig. 4.1: Diagram of 12" scale low-boom concept inlet model used in the NASA Glenn Research Center wind tunnel testing. 

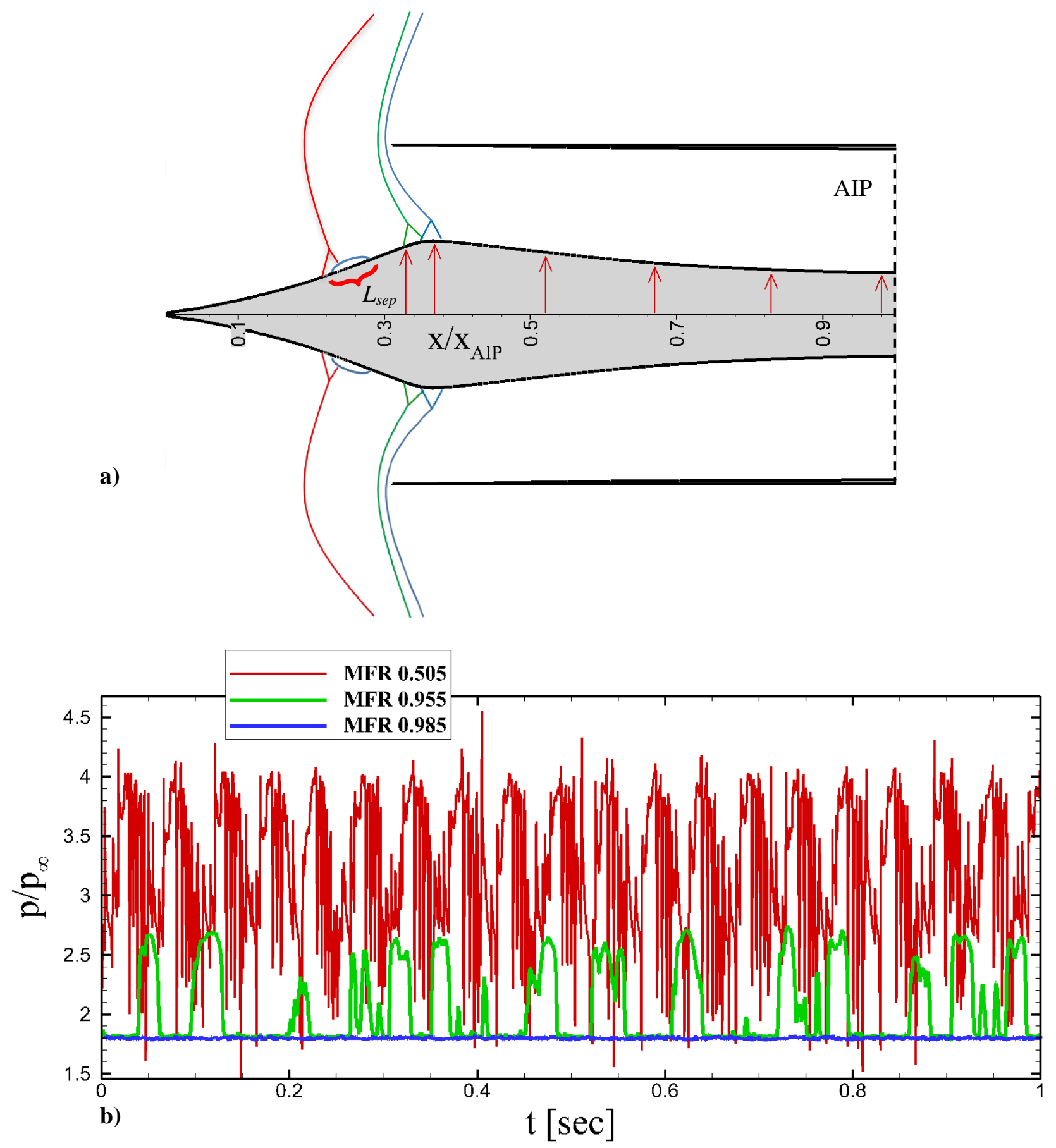

Fig. 4.2: Inlet conditions while operating at near-buzz 0.505 MFR (red), near-design 0.955 MFR (green), and on-design 0.985 MFR (blue) showing: a) the inlet model spanning from the centerbody tip to the AIP with illustrations of normal shock position, the lambda shock produced by the SWBLI and its corresponding downstream flow separation bubble characterizing the separation length scale. Red arrows indicate Kulite surface pressure tap streamwise location. b) Pressure traces of the upstream most tap located at $x / x_{A I P}=0.33$ which shows high amplitude oscillations at near-buzz, lower amplitude oscillations due to normal shock motion at neardesign, and low, steady supersonic pressure at on-design conditions. 

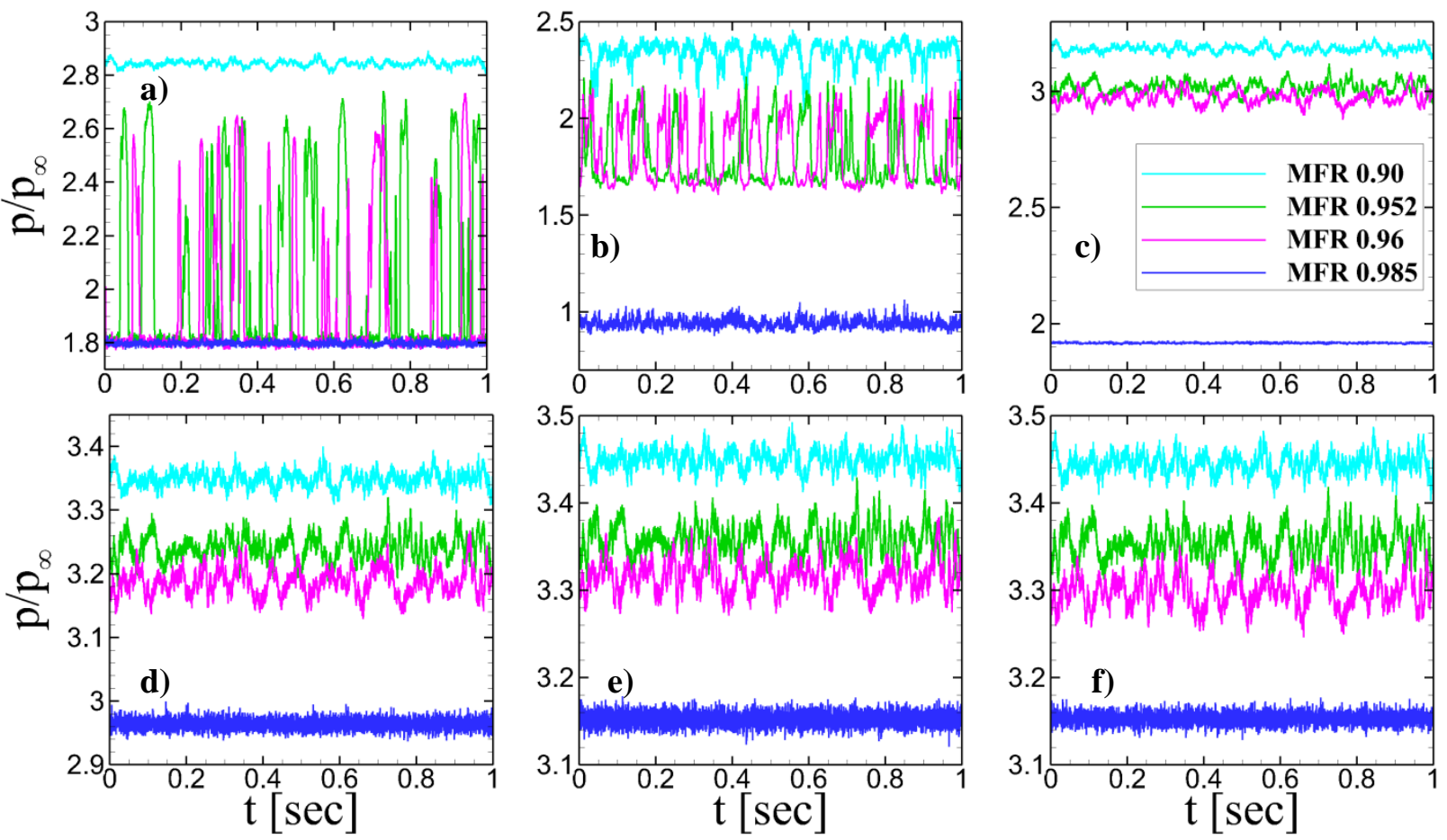

Fig. 4.3: Experimental pressure traces at four different MFR including the on-design 0.985 condition at $\left.x / x_{A I P}=\mathbf{a}\right) 0.33$, b) 0.37 , c) 0.52 , d) 0.67 , e) 0.83 , and f) 0.98 . 


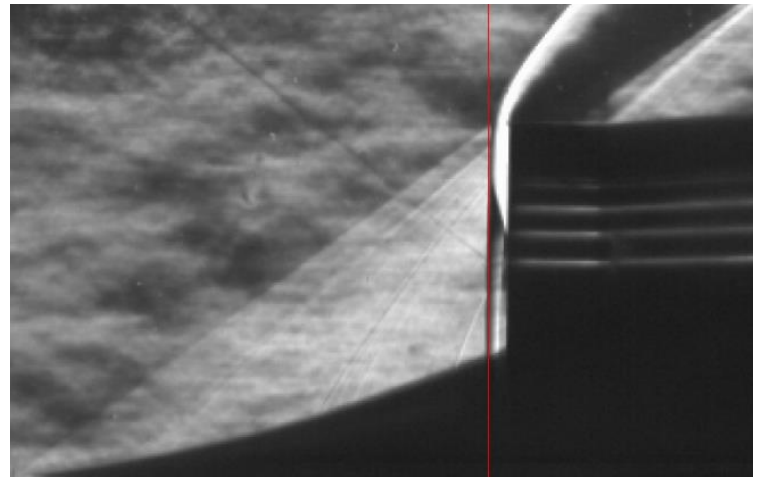

a)

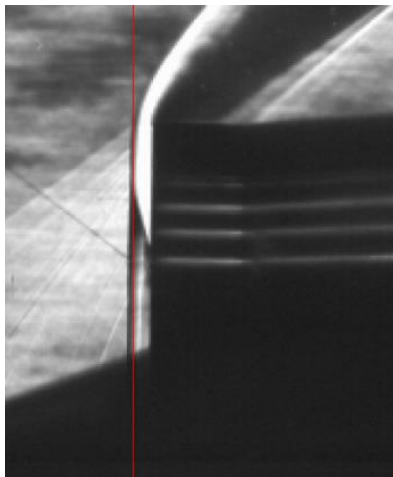

b)

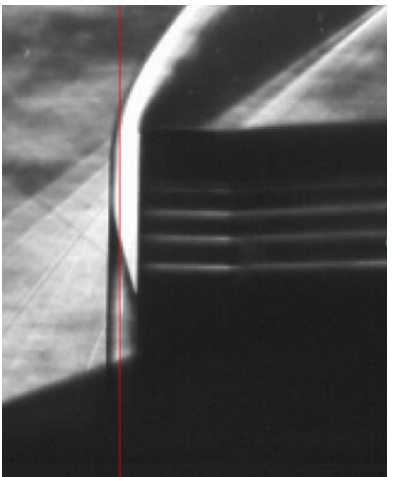

c)

Fig. 4.4: Still frames from schlieren video illustrating shock oscillations at an experimental MFR of 0.955. A red vertical line is superimposed to represent an average position. The shock can be seen a) downstream, b) at the average, c) and upstream of its average streamwise position. 
a)

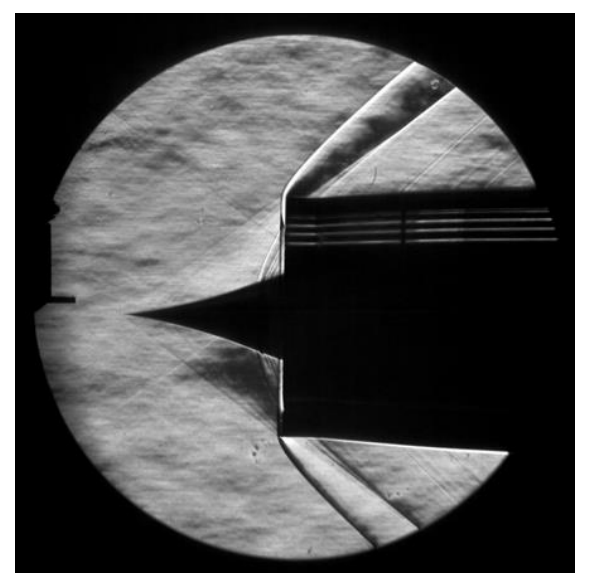

b)

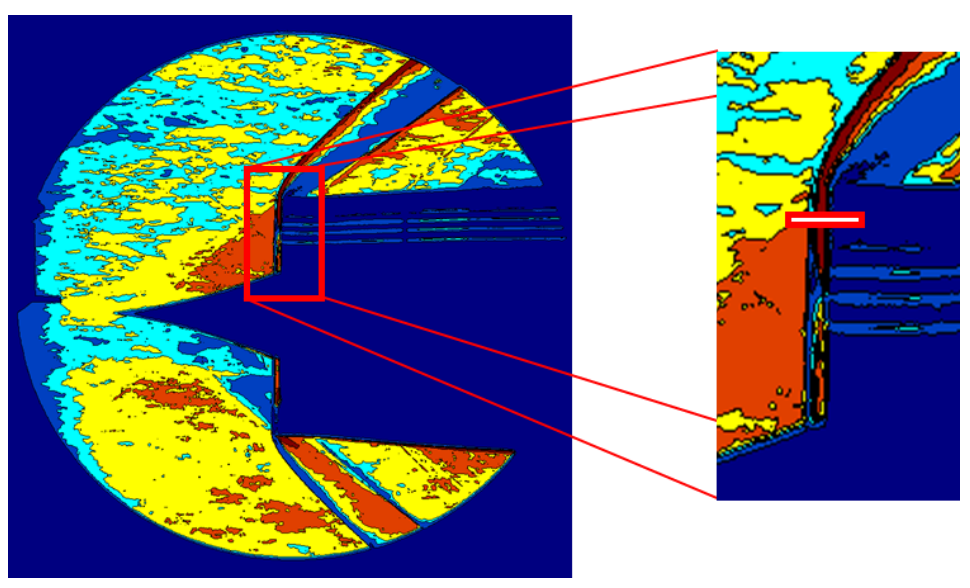

c)



Fig. 4.5: The process of extracting normal shock position using schlieren video from Rybalko ${ }^{2}$. a) The schlieren frames are converted into b) intensity matrices. c) Line distributions extracted from near the cowl reveal both the location of the normal shock and the cowl lip, where the difference between the two defines the shock stand-off distance. 

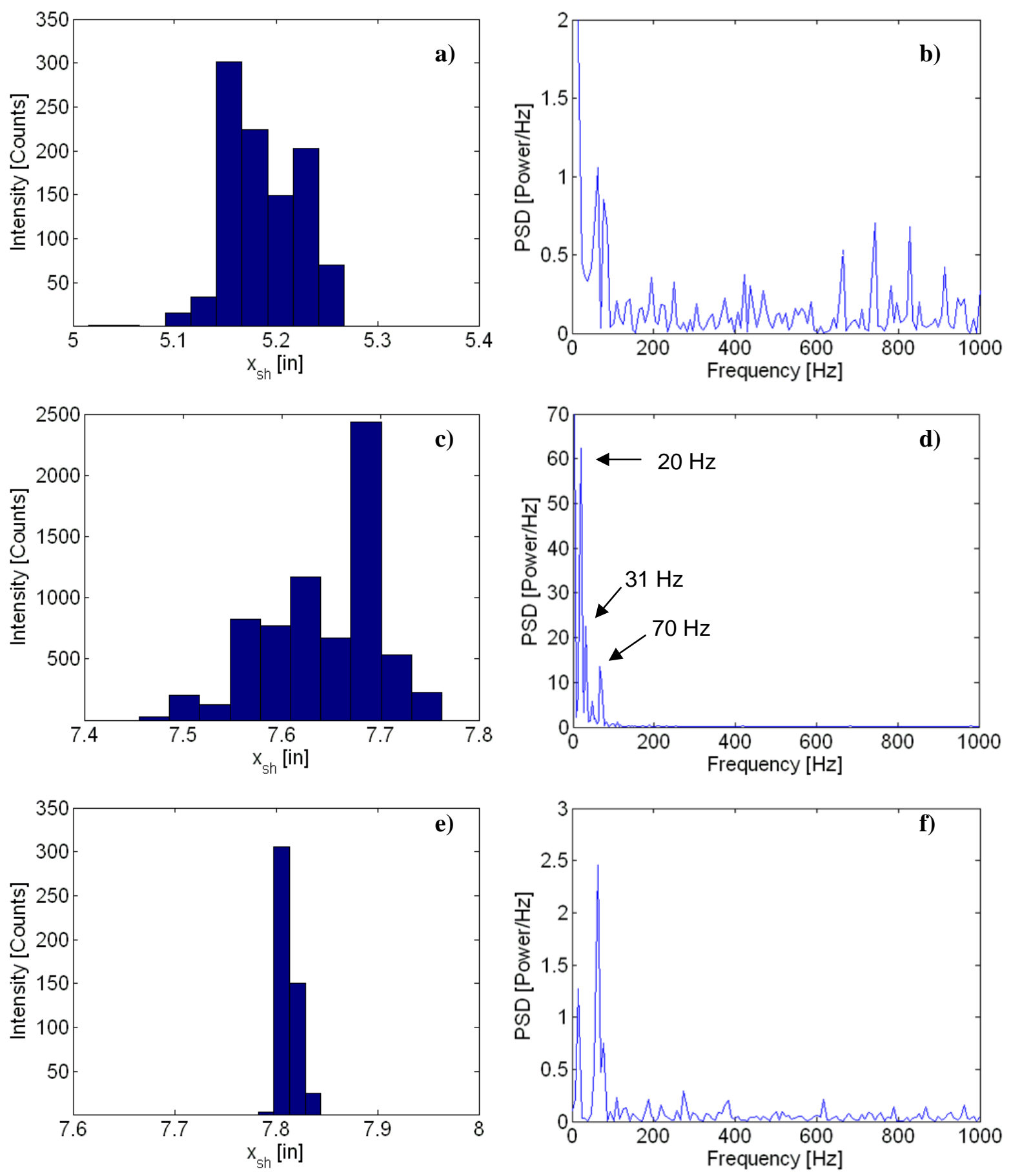

Fig. 4.6: Shock position histograms and power spectral density plots Rybalko ${ }^{2}$ at a), b) MFR = $0.505, \mathbf{c}), \mathbf{d}) \mathrm{MFR}=0.955$, and e), f) MFR $=0.985$. 

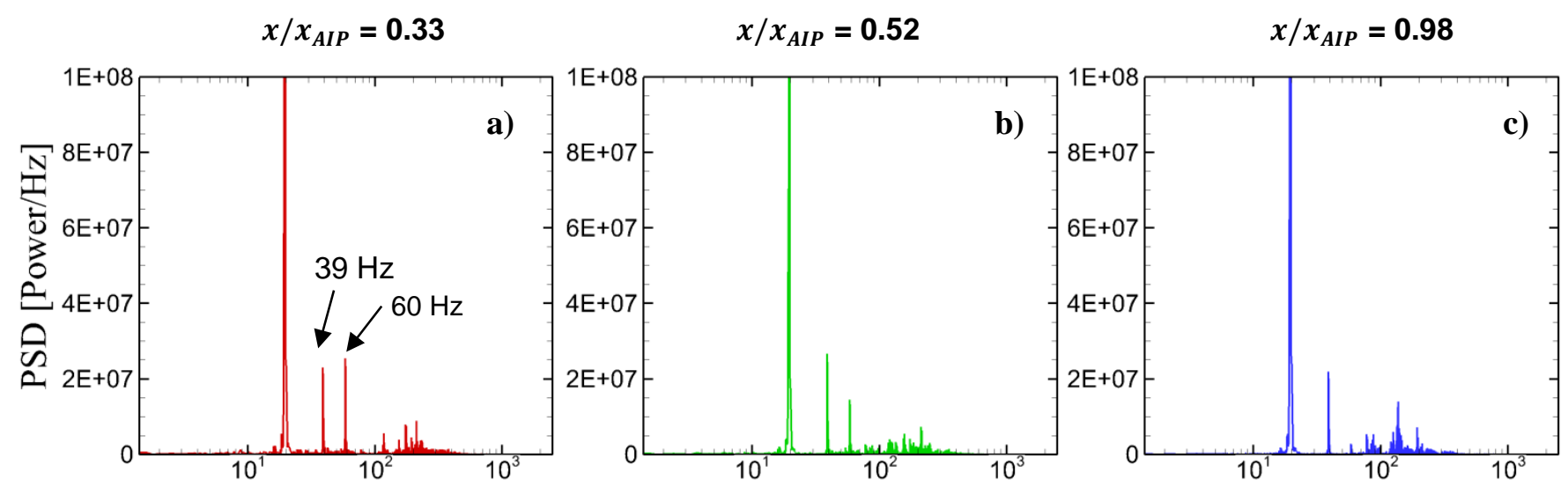

MFR

0.505


MFR

0.952
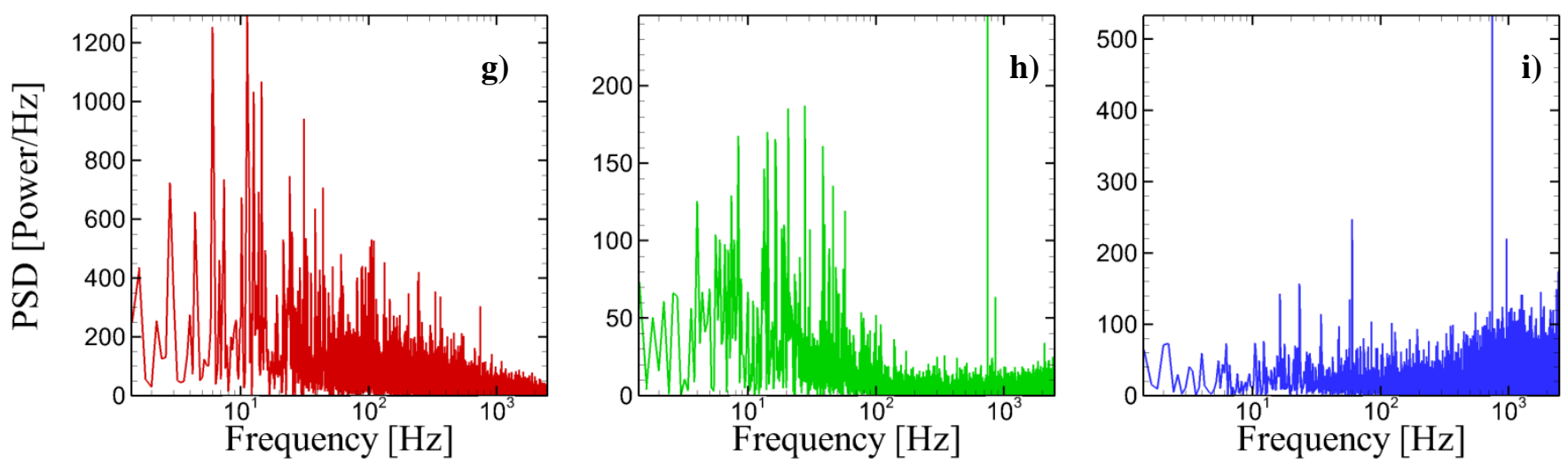

MFR

0.985

Fig. 4.7: Power spectral density plots of pressure traces at: a), b), c) $M F R=0.505$, d), e), f) MFR $=0.952$, and $\mathbf{g}), \mathbf{h})$, i) MFR $=0.985$ at streamwise locations of a), d), g) $x / x_{A I P}=0.33$ (red), b), e), h) $x / x_{A I P}=0.52$ (green) and c), f), i) $x / x_{A I P}=0.98$ (blue). 

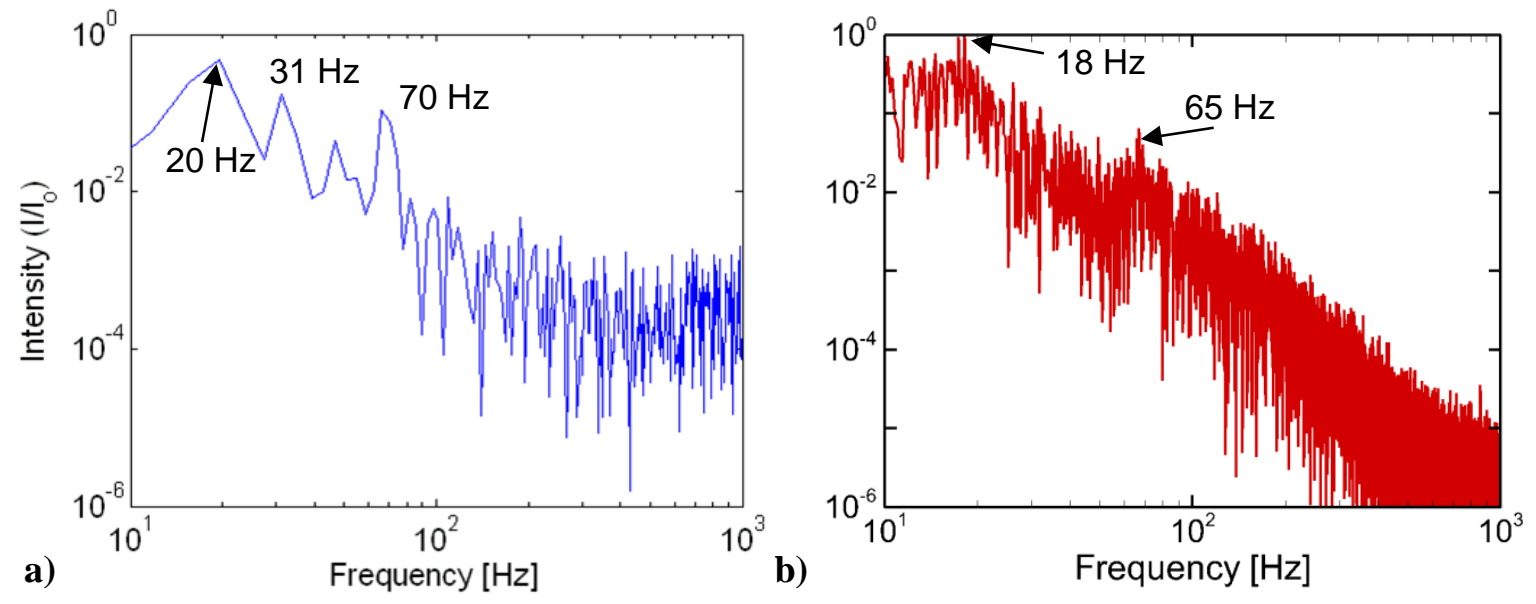

Fig. 4.8: Log-log plots of normalized power spectral density up to the Nyquist frequency of the schlieren frame rate for the near-design MFR for: a) 0.955 using shock tracking and b) 0.952 using unsteady surface pressure at $x / x_{A I P}=0.33$. 


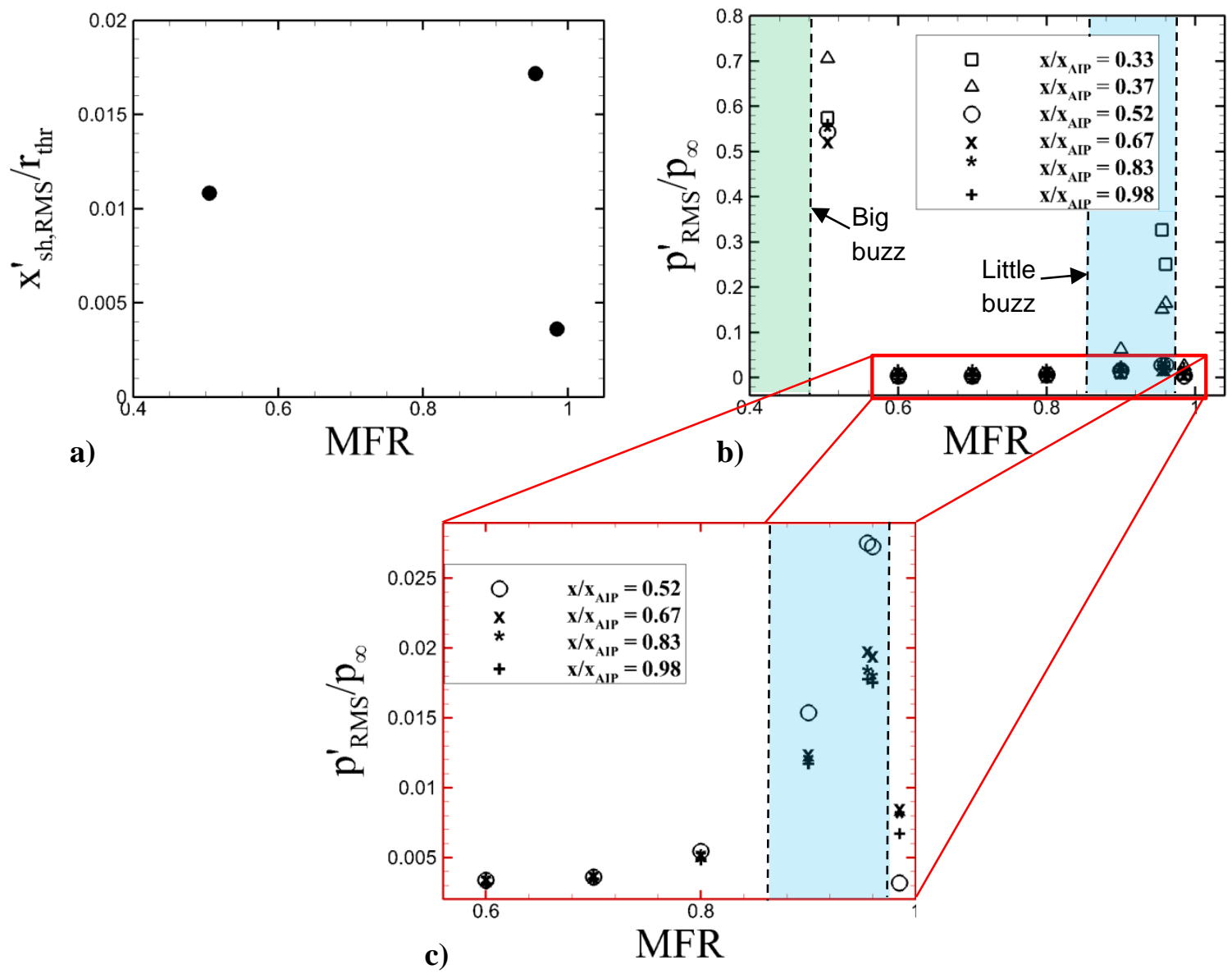

Fig. 4.9: Inlet buzz regimes are defined as a function of MFR using: a) normalized streamwise shock oscillations represented by the RMS values (from Rybalko ${ }^{2}$ ) and b) pressure fluctuation RMS values. Streamwise tap location with estimated big and little buzz regimes are denoted with green and blue regions, respectively. A red box illustrates a zoomed region seen in c) which focuses on the downstream pressure tap location for a range of MFRs. 


\section{Chapter 5: Summary and Recommendations}

A Detached Eddy Simulation approach was used to generate time-accurate numerical solutions to a low-boom axisymmetric external compression inlet flow-field. Comparisons are made between the numerical solution and an extensive experimental data set which show reasonable qualitative and quantitative agreement between various flow features. An emphasis is placed on the unsteady aspects of the flow, where the DES exhibits high sensitivity to computational domain length. Significant normal shock streamwise oscillations are seen only when using a computational domain that extends downstream to the second throat at the mass flow plug. A shorter domain utilizing a fixed downstream back pressure fails to produce similar shock oscillations, indicating a strong influence of reflecting compression waves on flow-field unsteadiness. Kulite pressure taps located along the inlet centerbody surface collect unsteady pressure measurements used to characterize the experimental flow unsteadiness. These Kulite taps can observe normal shock oscillations if the average normal shock position, which is highly dependent on mass flow rate, is just downstream of a tap. The normal shock and the Kulite taps are located such that at a near-design mass flow ratio of 0.96 (4\% spillage) the oscillations of the normal shock are captured. Propagation of compression waves can be monitored by tracking local pressure peaks throughout the unsteady pressure traces between streamwise stations. The propagation velocity of these peaks details the nature of these waves.

Compression waves are observed by local pressure peaks in the surface pressure time traces, clearly beginning in time at the downstream locations and propagating upstream. These upstream propagating waves are indicated in both experiments and DES. Reasonable agreement is seen between the wave propagation and the DES predicted upstream acoustic propagation, indicating the compression waves are acoustic in nature. Evidence of a second shock at the 
geometric throat is seen in a detailed investigation of the experimental pressure traces and is echoed by the DES. The mechanics of the compression wave are extracted from DES contours illustrating the upstream propagation which is largely diverted around the transonic region surrounding the throat shock. An expansion region just upstream of the throat shock caused by flow turning creates supersonic flow that isolates the throat region from being directly affected by the acoustic wave. However, motion of the normal shock alters the incoming throat boundary layer and produces increased unsteadiness in the throat region. The DES methodology has difficulty predicting the coupled dynamics of the two shocks and the separation bubbles produced by the shock wave boundary layer interactions. This leads to reduced unsteadiness likely caused by over-predicted flow mixing and turbulent dissipation in the RANS-solved portion of the flow near the reattaching region between the two shocks, which appears to be exacerbated with increasing grid resolution.

The effects of mass flow rate on flow-field unsteadiness are investigated at on-design, near-design, and near-buzz mass flow ratios of approximately 0.985, 0.955, and 0.505, respectively. The near-buzz case exhibits the greatest surface pressure unsteadiness but the greatest shock unsteadiness was seen in the near-design case. This suggests that the inlet experiences the little buzz phenomena while at near-design conditions. The on-design case proved to be the most stable with, in some cases, several orders of magnitude less power density of the fluctuating flow values. Spectral analysis using normal shock location and unsteady surface pressure traces generally show inconclusive results for identifying the dominant instabilities. Estimated frequencies due to the characteristic separation length behind the normal shock did not show significant power density, indicating that separation does not dominate flow unsteadiness at these three mass flow rates. A $20 \mathrm{~Hz}$ frequency wave clearly dominates the 
pressure fluctuations for both the near-design and near-buzz cases. The estimated acoustic frequency based on the acoustic Strouhal number by Ryablko ${ }^{2}$ and Dussauge ${ }^{3}$ is substantially greater than this observed $20 \mathrm{~Hz}$. However, the unsteady pressure traces indicate approximately 100 normal shock oscillations in a five second span equating to a $20 \mathrm{~Hz}$ frequency. This suggests that the $20 \mathrm{~Hz}$ frequency is likely acoustically driven based on the coupling between upstream running acoustic waves and normal shock oscillations seen in Chapters 2 and 3.

Further investigation of the dynamics and interactions of the normal and throat shocks at near-design conditions is recommended for future studies. Computational tools will likely provide the most detail as the throat region is difficult to characterize experimentally. Improved predictions of the boundary layer reattachment between the normal shock and the throat shock should be attempted. Using the current DES approach, modifications to the current grid cell distribution in this region could encourage additional LES resolved turbulence to better predict the flow dynamics. A higher fidelity approach such as pure LES will likely shed light on the physical interactions at the throat, but will drastically increase computational expense. A substantial increase in time-integration length is recommended to investigate the repeatability of the compression waves and to better compare with the five seconds of experimental data. Understanding the flow unsteadiness and identifying the instabilities may lead to improved or new flow control methods to increase external compression inlet performance and robustness. 


\section{References}

${ }^{1}$ Conners, T. R., and Howe, D. C., "Supersonic Inlet Shaping For Dramatic Reduction in Drag and Sonic Boom Strength," AIAA Paper, 2006-30.

${ }^{2}$ Rybalko, M., "Numerical and Experimental Investigation of VG Flow Control for a Low-Boom Inlet," Ph.D Dissertation 2011.

${ }^{3}$ Dussauge, J-P., Dupont, P., and Debiève, J-F., "Unsteadiness in Shock Wave Boundary Layer Interactions with Separation."Aerospace Science and Technology, Vol. 10, No. 2, 2006, pp. 85-91.

${ }^{4}$ Fukuda, M. K., Hingst, W. G., and Reshotko, E., "Control of Shock Wave-Boundary Layer Interactions by Bleed in Supersonic Mixed Compression Inlets,” NASA CR-2595, August 1975.

${ }^{5}$ Holden, H., and Babinsky, H., "Effect of Microvortex Generators on Separated Normal Shock/Boundary Layer Interactions," Journal of Aircraft, Vol. 44, No. 1, 2006, pp. 170-174.

${ }^{6}$ Hirt, S., Chima, R., Vyas, M., Wayman, T., Conners, T., and Reger, R., "Experimental Investigation of a Large-Scale Low-Boom Inlet Concept," $29^{\text {th }}$ AIAA Applied Aerodynamics Conference, Honolulu, HI, AIAA 2011-3796.

${ }^{7}$ Rybalko, M., Candon, S., and Loth, E., "Pressure and Shock Dynamics of a Low-Boom Inlet," $51^{s t}$ AIAA Aerospace Sciences Meeting, Grapevine, TX, 2013-0015.

${ }^{8}$ Rybalko, M., Loth, E., Chima, R. V., Hirt, S. M., and DeBonis, J. R., "Micro-Ramps for External Compression Low-Boom Inlets," AIAA Fluid Dynamics Conference, San Antonio, TX, AIAA 2009-4206.

${ }^{9}$ Gillen, T., Loth, E., and Rybalko, M., "Vortex Generators for Diffuser of Axisymmetric Supersonic Inlets," $5^{\text {th }}$ AIAA Flow Control Conference, Chicago, IL, AIAA 2010-4253.

${ }^{10}$ Rybalko, M., and Loth, E., "Vortex Generators for a Single-Stream Low-Boom Inlet," $29^{\text {th }}$ AIAA Applied Aerodynamics Conference, Honolulu, HI, AIAA 2011-3803.

${ }^{11}$ Rybalko, M., and Loth, E., "Detached Eddy Simulations of a Low-Boom Inlet," $50^{\text {th }}$ AIAA Aerospace Sciences Meeting, Nashville, TN, AIAA 2012-0015. 
${ }^{12}$ Lee, S., "Large Eddy Simulation of Supersonic Boundary Layer Interaction Control using MicroVortex Generators," Ph.D. Dissertation, November, 2009.

${ }^{13}$ Nichols, R. H., and Nelson, C. C., "Application of Hybrid RANS/LES Turbulence Models," $41^{s t}$ AIAA Aerospace Sciences Meeting, Reno, NV, AIAA 2003-83.

${ }^{14}$ Rybalko, M., Loth, E., and Lankford, D., "LES Sub-Grid Diffusion for Lagrangian Particles." ASME 2008 Fluids Engineering Division Summer Meeting collocated with the Heat Transfer, Energy Sustainability, and 3rd Energy Nanotechnology Conferences. American Society of Mechanical Engineers, 2008.

${ }^{15}$ Spalart, P. R., Deck, S., Shur, M. L., Squires, K. D., Strelets, M. Kh., and Travin, A., “A New Version of Detached-Eddy Simulation, Resistant to Ambiguous Grid Densities," Theoretical Computational Fluid Dynamics, Vol. 20, No. 3, 2006, pp. 181-195.

${ }^{16}$ Spalart, P. R., Jou, W.-H., Strelets, M., and Allmaras, S. R., "Comments on the Feasibility of LES for Winds, and on a Hybrid RANS/LES Approach," Proceedings of first AFOSR International conference on DNS/LES, Ruston, LA, Greyden Press, 1997.

${ }^{17}$ Fisher, S. A., Neale, M. C., and Brooks, A. J. "On the Sub-Critical Stability of Variable Ramp Intakes at Mach Numbers Around 2," National Gas Turbine Establishment Report No. ARC-R/M-3711, Feb. 1970.

${ }^{18}$ Ferri, A., and Nucci, L. M., "The Origin of Aerodynamic Instability of Supersonic Inlets at Subcritical Conditions,” NACA RM L50K30, 1951.

${ }^{19}$ Dailey, C. S., "Supersonic Diffuser Instability," Ph.D Dissertation, California Institute of Technology, 1954.

${ }^{20}$ Trapier, S., Duveau, P., and Deck, S., "Experimental study of supersonic inlet buzz." AIAA Journal, Vol. 44, No. 10, 2006, pp. 2354-2365.

${ }^{21}$ Culick, F. E. C., and Rogers, T., "The Response of Normal Shocks in Diffusers." AIAA Journal, Vol. 21, No. 10, 1983, pp. 1382-1390. 
${ }^{22}$ Liou, M-S., and Coakley, T. J., "Numerical Simulations of Unsteady Transonic Flow in Diffusers." AIAA Journal, Vol. 22, No.8, 1984, pp. 1139-1145.

${ }^{23}$ Robinet, J-C., and Casalis, G.. "Shock Oscillations in Diffuser Modeled by a Selective Noise Amplification." AIAA Journal, Vol. 37, No.4, 1999, pp. 453-459.

${ }^{24}$ Oh, J. Y., Ma,F., Hsieh, S. Y., and Yang, V., "Interactions Between Shock and Acoustic Waves in a Supersonic Inlet Diffuser." Journal of Propulsion and Power, Vol. 21, No.3, 2005, pp. 486-495.

${ }^{25}$ Bur, R., Benay, R., Galli, A., and Berthouze, P., "Experimental and numerical Study of Forced Shock-Wave Oscillations in a Transonic Channel." Aerospace Science and Technology, Vol. 10, No. 4, 2006, pp. 265-278.

${ }^{26}$ Rybalko, M., Holger B., and Loth, E., "Vortex Generators for a Normal Shock/Boundary Layer Interaction with a Downstream Diffuser." Journal of Propulsion and Power, Vol. 28, No.1, 2012, pp. 7182. 